\title{
INTERTWINING OPERATORS AND COMPOSITION SERIES OF GENERALIZED AND DEGENERATE PRINCIPAL SERIES FOR $S p(4, \mathbb{R})$
}

\author{
Goran Muić \\ University of Zagreb, Croatia
}

\begin{abstract}
In this paper we study analytic properties of intertwining operators and apply them to the determination of the composition series of degenerate and generalized principal series for $S p(4, \mathbb{R})$. We expect that that some of the methods developed here will extend to higher rank groups in order to extend the formalism of degenerate Eisenstein series given by [21] and [22]. In higher rank cases we expect to be more dependent on the algebraic theory of representation theory of the real reductive groups as developed by Vogan [30].
\end{abstract}

\section{INTRODUCTION}

In the theory of automorphic forms, and especially in the theory of Eisenstein series, (see $[6-9,13-16,21,22,26,32]$ ) it is important to have a good understanding of standard local intertwining operators: their poles, images and kernels. This is directly related to the structure of composition series of degenerate and generalized principal series of a given reductive group over a local field (Archimedean or not).

In the series of the papers ([18-20]) we have obtained relatively good understanding of the reducibility and composition series of generalized principal series for classical $p$-adic groups. Then one can apply Aubert-SchneiderSthuler-Zelevinsky involution [1] to obtain the reducibility and composition series of degenerate principal series. As an application of our work, we have constructed in a elegant way a large family of residual automorphic representations [21] that enables us to prove the unitarity of many isolated unitary

2000 Mathematics Subject Classification. 22E50.

Key words and phrases. Intertwining operators, composition series, generalized principal series. 
representations of $p$-adic, real and complex classical groups proving that they appear as local components of representations from the corresponding (residual part of) discrete spectrum (see $[21,23]$ ). This paper is a step towards extending our construction from [21] and [22] by relaxing the requirement that the archimedean component is spherical. In a more general case, we expect to use the same ideas to determine irreducible quotients and subrepresentations of certain degenerate representations in order to extend the formalism of Eisenstein series given by [21] and [22] in order to determine the appearance in the residual spectrum of $S p\left(2 n, \mathbb{A}_{\mathbb{Q}}\right)$ of irreducible representations for $S p(2 n, \mathbb{R})$. But in higher rank cases we expect to be more dependent on the algebraic theory of the representation theory of the real reductive groups as developed by Vogan [30].

The approach in the present paper is very conceptual and essentially analytic. Everything is based on the classification of irreducible Harish-Chandra modules as explained in the book [10]: the classification of tempered representations due to Knapp-Zuckerman and the Langlands quotient theorem (see Section 8 for the description of irreducible Harish-Chandra modules for $S p(4, \mathbb{R}))$. But we use analytic properties of intertwining operators extensively (see Lemmas 7.2 and 7.3 of Section 7 ), and in a rather novel way. Except that we use $K$-types (see Lemma 6.1 ) combined with the usual theory of translation functors $([10,33])$. As we pointed above, more things need to be added for higher rank groups.

We explain the content of the paper. In Section 2 we fix notation and recall some well-known results related to the classification of irreducible HarishChandra modules. In Section 3 we recall the translation functors $([10,33])$, and prove some technical results needed later in the paper (see Lemma 3.4 and Lemma 3.5). In Section 4 we compute translation functors for $G L_{2}(\mathbb{R})$ (see Lemmas 4.1 and 4.2) using the material of Section 3. In Section 5, we write down the reducibility of principal series (see Lemma 5.1). This follows from [27], but for the convenience of the reader we give a more direct proof combining the idea of the factorization of long-intertwining operators ([27]) with the computation of $R$-groups of Goldberg ([4]) (We should remark here that although the work of Goldberg is on $R$-groups for split classical groups $p$-padic, his work is based on general results on $R$-groups that holds also for real groups ([10]). Therefore, as it was remarked in the introduction of [4], the results of [4] hold also for real classical groups.). In Section 6, we compute $K$-type structure of principal, generalized and degenerate principal series (see Lemma 6.1). In Section 7, we elaborate our idea of using analytic properties of intertwining operators (Lemmas 7.2 and 7.3). This is the central section of the paper. We use Lemmas 7.2 and 7.3 as our main tool in studying the structure of generalized and degenerate principal series for $S p(4, \mathbb{R})$. In Section 8, we recall from [10] the classification of irreducible Harish-Chandra modules for $S p(4, \mathbb{R})$. In Sections 9, 10, and 11 we determine 
composition series of generalized and degenerate principal series for $S p(4, \mathbb{R})$ for integral infinitesimal character. In Section 12, we consider the easy case of non-integral infinitesimal character.

We should warn the reader that there are well-known approaches in the determination of composition series of generalized principal series for real reductive groups. One approach is based on usage of cohomological methods (see for example [11] and [30]). The other approach is based on geometric considerations (see for example [12]). In the case of $S p(4, \mathbb{R})$ they are rather different than the one explained here but one may use them (and some hard work) to write down the results of the present paper but without getting any information on the images and kernels of intertwining operators.

The structure composition series of generalized and degenerate principal series for $S p(4, F), F p$-adic, was obtained earlier by Sally and Tadić in [24]. Their approach is based on Jacquet modules.

This manuscript is an extension of the manuscript called "The composition series of generalized and degenerate principal series for $\operatorname{Sp}(2, \mathbb{R})$ ".

\section{PRELIMINARIES}

Let $H$ be a (Zariski connected) algebraic group defined over $\mathbb{R}$. We write $H(\mathbb{R})$ for the group of its $\mathbb{R}$-points. $H(\mathbb{R})$ has a structure of a Lie group and we write $\mathfrak{h}_{\mathbb{R}}$ for its Lie algebra. The complexification of $\mathfrak{h}_{\mathbb{R}}$ is $\mathfrak{h}_{\mathbb{C}}:=\mathfrak{h}_{\mathbb{R}} \otimes_{\mathbb{R}} \mathbb{C}$. It is a complex Lie algebra. Finally, $H(\mathbb{R})$ need not be connected in the metric topology. We write $H(\mathbb{R})^{0}$ for its identity component.

Let $G$ be a reductive split algebraic group defined over $\mathbb{R}$. Then the group of $\mathbb{R}$-points $G(\mathbb{R})$ has a natural structure of the real reductive group in the sense of [30]. We assume that $G$ is split over $\mathbb{R}$ and write $B=T U$ for its Borel subgroup, where $T$ is split torus and $U$ is maximal unipotent radical of $B$. We write $\Sigma$ for the set of roots of $G$ with respect to $T$ and $\Sigma_{+}$for the set of positive roots with respect to $B$. Let $\Delta$ be the basis of $\Sigma_{+}$. Let $W$ be the Weyl group of $G$ with respect to $T, W \simeq N_{G}(T) / T$. One can take representatives of elements in $W$ in $G(\mathbb{R})$.

Since $G$ is split over $\mathbb{R}$, this gives us the following decomposition on the level of Lie algebra:

$$
\mathfrak{g}_{\mathbb{R}}=\oplus_{\alpha \in \Delta_{+}}\left(\mathfrak{g}_{\mathbb{R}}\right)_{-\alpha} \oplus \mathfrak{t}_{\mathbb{R}} \oplus_{\alpha \in \Delta_{+}}\left(\mathfrak{g}_{\mathbb{R}}\right)_{\alpha}
$$

We know that $\operatorname{dim}_{\mathbb{R}}\left(\mathfrak{g}_{\mathbb{R}}\right)_{ \pm \alpha}=1$ and we may take a Chevalley basis $X_{ \pm \alpha}$. We know that there is a unique antilinear-automorphism of order two $\theta$ of $G(\mathbb{C})$ such that its differential on $\mathfrak{g}_{\mathbb{C}}$ satisfies $d \theta\left(X_{\alpha}\right)=-X_{-\alpha}$ and $\left.d \theta\right|_{\mathfrak{t}(\mathbb{R})}=-i d$. The group of fixed points $G(\mathbb{C})^{\theta}$ define maximal compact subgroup of $G(\mathbb{R})$ by $K_{\mathbb{R}}=G(\mathbb{R}) \cap G(\mathbb{C})^{\theta}$ so that we have the Iwasawa decomposition $G(\mathbb{R})=$ $K_{\mathbb{R}} \cdot T(\mathbb{R})^{0} \cdot U(\mathbb{R})$. 
For any subset $\Theta \subseteq \Delta$, there is a standard parabolic subgroup $P_{\Theta}=P=$ $M N \subseteq G$ (Levi decomposition), where $A=\left(\cap_{\alpha \in \Theta} \operatorname{ker} \alpha\right)^{0}{ }^{1}$ and $M=Z_{G}(A)$. $A$ is a maximal split torus in the center of $M$. We write $\Sigma_{+}^{\Theta}$ for the set of all positive roots of $T$ in $M$ with respect to $B \cap M$. Next, $X(M)$ and $X(A)$ are the groups of ( $\mathbb{R}$-rational) characters of $M$ and $A$, respectively. Then $X(M) \subseteq X(A)$ is a subgroup of finite index. Hence

$$
X(M) \otimes_{\mathbb{Z}} \mathbb{R} \simeq X(A) \otimes_{\mathbb{Z}} \mathbb{R}:=\mathfrak{a}_{\mathbb{R}}^{*}
$$

Put $\mathfrak{a}_{\mathbb{R}}:=\operatorname{Hom}\left(\mathfrak{a}_{\mathbb{R}}^{*}, \mathbb{R}\right)$. As notation suggests, it can be identified by the Lie algebra of $A(\mathbb{R})$. We write $\langle$,$\rangle for the canonical pairing \mathfrak{a}_{\mathbb{R}}^{*} \times \mathfrak{a}_{\mathbb{R}} \rightarrow \mathbb{R}$. We define (smooth) homomorphism $H_{M}: M(\mathbb{R}) \rightarrow \mathfrak{a}_{\mathbb{R}}$ characterized as follows:

$$
\exp \left\langle\chi, H_{M}(m)\right\rangle=|\chi(m)|, \quad \forall \chi \in X(M) .
$$

Clearly, any $\nu \in \mathfrak{a}_{\mathbb{C}}^{*}=\mathfrak{a}_{\mathbb{R}}^{*} \otimes_{\mathbb{R}} \mathbb{C}$, defines a character as follows:

$$
m \rightsquigarrow \exp \left\langle\nu, H_{M}(m)\right\rangle \text {. }
$$

Its differential is $\nu$. Put ${ }^{0} M=\operatorname{ker} H_{M}$. Then we clearly have $M(\mathbb{R})=$ ${ }^{0} M \cdot A(\mathbb{R})^{0}$ (direct product). Thus, Levi decomposition is more traditionally written as a Langlands decomposition $P(\mathbb{R})={ }^{0} M \cdot A(\mathbb{R})^{0} \cdot N(\mathbb{R})$ (direct product of smooth manifolds).

This of course applies to $G\left(=P_{\Delta}\right)$ itself. If $Z$ is maximal $\mathbb{R}$-split subtorus of the center of $G$, then $G(\mathbb{R})={ }^{0} G \cdot Z(\mathbb{R})^{0}$.

Let $G=G L_{n}$ so that $G(\mathbb{R})=G L_{n}(\mathbb{R})$. Let $Z$ be the center of $G L_{n}$. Then $\nu \in \mathfrak{z}_{\mathbb{C}}^{*} \simeq \mathbb{C}$, and we have $\exp \left\langle\nu, H_{G L_{n}}(g)\right\rangle=|\operatorname{det} g|^{\nu}$.

If $\Theta=\emptyset$, then $P=B, N=U$, and $T=M=A$. In this case we also have $T(\mathbb{R})={ }^{0} T \cdot T(\mathbb{R})^{0}$. In this case ${ }^{0} T$ is just a finite group that can be characterized as follows ${ }^{0} T=K_{\mathbb{R}} \cap T(\mathbb{R})$. We have an embedding $\mathfrak{a}_{\mathbb{R}}^{*} \hookrightarrow \mathfrak{t}_{\mathbb{R}}^{*}$, defined by restriction of characters:

$$
\exp \left\langle\nu, H_{M}(t)\right\rangle=\exp \left\langle\nu, H_{T}(t)\right\rangle \quad \forall t \in T .
$$

We define the positive Weyl chamber $\mathcal{C}^{+}(A, N)$ of $A$ with respect to $N$ as the set of all $\nu \in \mathfrak{a}_{\mathbb{R}}^{*}$ such that

$$
\left\langle\nu, \alpha^{\vee}\right\rangle>0, \quad \forall \alpha \in \Sigma_{+} \backslash \Sigma_{+}^{\Theta} .
$$

Finally, let $\nu_{1}, \nu_{2} \in \mathfrak{t}_{\mathbb{R}}^{*}$. Then we write $\nu_{1} \geq \nu_{2}$, if $\nu_{1}-\nu_{2}=\sum_{\alpha \in \Delta_{+}} c_{\alpha} \alpha$, where all $c_{\alpha} \in \mathbb{R}_{\geq 0}$.

We write $\operatorname{Irr}(G(\mathbb{R}))$ for the set of equivalence classes of irreducible (admissible) $\left(\mathfrak{g}_{\mathbb{R}}, K_{\mathbb{R}}\right)$-modules. We write $\operatorname{Irr}(G(\mathbb{R}))^{\text {temp }}$ and $\operatorname{Irr}(G(\mathbb{R}))^{\text {disc }}$ for subclasses of tempered and discrete series (admissible) $\left(\mathfrak{g}_{\mathbb{R}}, K_{\mathbb{R}}\right)$ modules. When $\left(\mathfrak{g}_{\mathbb{R}}, K_{\mathbb{R}}\right)$-module is unitarizable, it has a up to unitary equivalence unique globalization to the irreducible unitary representation of $G(\mathbb{R})$; we denote it by $\mathcal{H}_{G}(X)$ or just $\mathcal{H}(X)$. We write $\mathcal{R}(G(\mathbb{R}))$ for the Grothendieck group of finite length admissible $\left(\mathfrak{g}_{\mathbb{R}}, K_{\mathbb{R}}\right)$ - modules.

\footnotetext{
${ }^{1}$ Here superscript 0 means a connected component in an algebraic sense.
} 
We also recall Harish-Chandra's subquotient theorem and the description of infinitesimal characters. Let $Z\left(\mathfrak{g}_{\mathbb{C}}\right)$ be the center of universal enveloping algebra $\mathcal{U}\left(\mathfrak{g}_{\mathbb{C}}\right)$. If $\gamma: Z\left(\mathfrak{g}_{\mathbb{C}}\right) \rightarrow \mathcal{U}\left(\mathfrak{t}_{\mathbb{C}}\right)^{W}$ is Harish-Chandra homomorphism, then for $\lambda \in t_{\mathbb{C}}^{*}$, we define character $\chi_{\lambda}$ of $Z\left(\mathfrak{g}_{\mathbb{C}}\right)$. We have $\chi_{\lambda}=\chi_{\lambda^{\prime}}$ if and only if $\lambda=w\left(\lambda^{\prime}\right)$, for some $w \in W$. If $X \in \operatorname{Irr}(G(\mathbb{R}))$, then $Z\left(\mathfrak{g}_{\mathbb{C}}\right)$ acts by $\chi_{\lambda}$ on $X$, for some $\lambda$. Shortly, we say that $X$ has an infinitesimal character $\lambda$.

TheOREM 2.1. Let $\mu: T(\mathbb{R}) \rightarrow \mathbb{C}^{\times}$be a character. We can write in a more traditional way $(\delta, \nu)$, according to the decomposition $T(\mathbb{R})={ }^{0} T \cdot T(\mathbb{R})^{0}$, where $\delta=\left.\mu\right|_{o_{T(\mathbb{R})}}$ and $\exp \left\langle\nu, H_{T}(\cdot)\right\rangle=\left.\mu\right|_{T(\mathbb{R})^{0}}$. We have the following:

(i) Two principal series $\operatorname{Ind}_{B(\mathbb{R})}^{G(\mathbb{R})}(\mu)_{K_{\mathbb{R}}-\text { finite }}$ and $\operatorname{Ind}_{B(\mathbb{R})}^{G(\mathbb{R})}\left(\mu^{\prime}\right)_{K_{\mathbb{R}}-\text { finite }}$ have the same semi-simplification in $R(G(\mathbb{R}))$ if and only if there exists $w \in W$ such that $w(\mu)=\mu^{\prime}$ or, equivalently, $w(\delta)=\delta^{\prime}$ and $w(\nu)=\nu^{\prime}$.

(ii) All irreducible subquotients of the principal series $\operatorname{Ind}_{B(\mathbb{R})}^{G(\mathbb{R})}(\mu)_{K_{\mathbb{R}} \text {-finite }}$ have the same infinitesimal character $\chi_{\nu}$.

(iii) Assume that $X$ is an irreducible $\left(\mathfrak{g}_{\mathbb{R}}, K_{\mathbb{R}}\right)$-module with infinitesimal character $\chi_{\lambda}$. Then there exists character $\mu: T(\mathbb{R}) \rightarrow \mathbb{C}^{\times}$, $\exp \left\langle\lambda, H_{T}(\cdot)\right\rangle=\left.\mu\right|_{T(\mathbb{R})^{0}}$, such that $X$ is an irreducible subquotient of $\operatorname{Ind}_{B(\mathbb{R})}^{G(\mathbb{R})}(\mu)_{K_{\mathbb{R}}-\text { finite }}$.

If $G(\mathbb{R})=G L_{n}(\mathbb{R})$ or $G(\mathbb{R})=S p(2 n, \mathbb{R})$, then we write $\mu=\mu_{1} \otimes \cdots \otimes \mu_{n}$, and we write following Tadic and Zelevinsky (cf. [T, Ze]):

$$
\begin{aligned}
& \mu_{1} \times \cdots \times \mu_{n}:=\operatorname{Ind}_{B(\mathbb{R})}^{G(\mathbb{R})}(\mu)_{K_{\mathbb{R}}-\text { finite }}, \quad G(\mathbb{R})=\underset{n}{G L(\mathbb{R})} ; \\
& \mu_{1} \times \cdots \times \mu_{n} \rtimes \mathbf{1}:=\operatorname{Ind} d_{B(\mathbb{R})}^{G(\mathbb{R})}(\mu)_{K_{\mathbb{R}}-\text { finite }}, \quad G(\mathbb{R})=S p(2 n, \mathbb{R}) .
\end{aligned}
$$

We can parameterize all characters of $\mathbb{R}^{\times}$as follows. Put $\operatorname{sgn}(x)=x /|x|$. This is a character of $\mathbb{R}^{\times}$and all other can be obtain are of the form $\operatorname{sgn}()^{\epsilon}||^{s}$, $s \in \mathbb{C}, \epsilon \in\{0,1\}$. Thus, we can write $\mu_{i}=\operatorname{sgn}()^{\epsilon_{i}}||^{s_{i}}, s_{i} \in \mathbb{C}, \epsilon_{i} \in\{0,1\}$. Then in both cases in (2.1) the infinitesimal character is given by $\left(s_{1}, \ldots, s_{n}\right) \in$ $\mathbb{C}^{n}$.

More generally, we have the following theorem

Theorem 2.2. Let $P=M N$ be a standard parabolic subgroup of $G$ where $G$ is a split reductive $\mathbb{R}$-group. Let $\mathcal{H}_{M}$ be some irreducible admissible Hilbert space representation of $M$ having infinitesimal character $\chi_{\lambda}$. Assume $\nu \in \mathfrak{a}_{\mathbb{C}}^{*}$. Then any irreducible subquotient of $\operatorname{Ind}_{P(\mathbb{R})}^{G(\mathbb{R})}\left(\exp \left\langle\nu, H_{M}(\cdot)\right\rangle \otimes \mathcal{H}_{M}\right)_{K_{\mathbb{R}}-\text { finite }}$ has infinitesimal character $\chi_{\lambda+\nu}$

Finally, we recall the Langlands classification.

Theorem 2.3. Let $P$ be a standard parabolic subgroup of $G, X_{M}$ a tempered irreducible $\left(\mathfrak{m}_{\mathbb{R}}, K_{\mathbb{R}} \cap M(\mathbb{R})\right)$-module, and $\nu \in \mathcal{C}^{+}(A, N)$. Then the 
$\left(\mathfrak{g}_{\mathbb{R}}, K_{\mathbb{R}}\right)$-module on

$$
\operatorname{Ind}_{P(\mathbb{R})}^{G(\mathbb{R})}\left(\exp \left\langle\nu, H_{M}(\cdot)\right\rangle \otimes \mathcal{H}\left(X_{M}\right)\right)_{K_{\mathbb{R}}-\text { finite }}
$$

has a unique irreducible quotient. We write

$$
\operatorname{Lang}\left(\operatorname{Ind}_{P(\mathbb{R})}^{G(\mathbb{R})}\left(\exp \left\langle\nu, H_{M}(\cdot)\right\rangle \otimes \mathcal{H}\left(X_{M}\right)\right)\right)
$$

for the Langlands quotient. We call an induced representation of the form (2.2) a standard representation. The Langlands quotient appears with multiplicity one in the composition series of (2.2). Let $w_{l}$ be the longest element of $W$ and let $w_{l, \Theta}$ be the longest element of the Weyl group of $\Theta$. Then $w_{l} w_{l, \Theta}(\Theta) \subseteq \Delta$. We write $P^{\prime}=M^{\prime} N^{\prime}$ for the corresponding standard parabolic subgroup. Then we again form induced representation:

$$
\operatorname{Ind}_{P^{\prime}(\mathbb{R})}^{G(\mathbb{R})}\left(\exp \left\langle w_{l} w_{l, \Theta}(\nu), H_{M}(\cdot)\right\rangle \otimes w_{l} w_{l, \Theta}\left(\mathcal{H}\left(X_{M}\right)\right)\right)_{K_{\mathbb{R}}} \text {-finite }
$$

Then the induced representations (2.2) and (2.3) have the same semisimplifications in $\mathcal{R}(G(\mathbb{R}))$. The semisimplification of standard module in (2.2) in $\mathcal{R}(G(\mathbb{R}))$ is of the form

$$
\begin{aligned}
& \operatorname{Ind}_{P(\mathbb{R})}^{G(\mathbb{R})}\left(\exp \left\langle\nu, H_{M}(\cdot)\right\rangle \otimes \mathcal{H}\left(X_{M}\right)\right)_{K_{\mathbb{R}}-\text { finite }}= \\
& \operatorname{Lang}\left(\operatorname{Ind}_{P(\mathbb{R})}^{G_{n}(\mathbb{R})}\left(\exp \left\langle\nu, H_{M}(\cdot)\right\rangle \otimes H\left(X_{M}\right)\right)+\sum_{X \in \operatorname{Irr} G(\mathbb{R}), \nu_{X}<\nu} m(X) \cdot X,\right.
\end{aligned}
$$

where $m(X) \in \mathbb{Z}_{\geq 0}$, and it is zero for all except finitely many $X \in \operatorname{Irr}(G(\mathbb{R}))$. Moreover, the Langlands quotient is a unique irreducible subrepresentation in (2.3). Next, the space of intertwining operators between the induced representations (2.2) and (2.3) is one-dimensional. Finally, if $X \in \operatorname{Irr}(G(\mathbb{R}))$, then there exists a unique standard parabolic subgroup $P=P_{\Theta}$, a unique tempered irreducible $\left(\mathfrak{m}, K_{\mathbb{R}} \cap M(\mathbb{R})\right)$-module $X_{M}$, and a unique $\nu \in \mathcal{C}^{+}(A, N)$ such that $X$ is equivalent to the Langlands quotient of (2.2).

The next two theorems are well-known.

TheOREM 2.4. Let $G(\mathbb{R})=S L_{2}(\mathbb{R})$. Then $K_{\mathbb{R}}=U(1)$. We write the Weyl group $W=\left\{i d, w_{0}\right\}$ of $T$ in $G$. Put $\eta=s g n^{\epsilon}||^{s}, s \in \mathbb{C}, \epsilon \in\{0,1\}$. Then we have the following:

(i) $\eta \rtimes \mathbf{1}$ is irreducible unless $s \in \mathbb{Z}$ and $\epsilon \equiv(s+1)(\bmod 2)$.

(ii) Let $s \in \mathbb{Z}_{>0}$. Then there are two non-equivalent representations in the discrete series $X(s,+)$ (of lowest $K_{\mathbb{R}}$-type $s+1$ ) and $X(s,-)$ (of highest $K_{\mathbb{R}}$-type $\left.-s-1\right)$, and a unique irreducible finite-dimensional representation of dimension $s, V_{s}$ (it has a highest weight $s-1$ ) such that:

$$
\begin{aligned}
& X(s,+) \oplus X(s,-) \hookrightarrow||^{s} \operatorname{sgn}^{s+1} \rtimes \mathbf{1} \rightarrow V_{s}, \\
& V_{s} \hookrightarrow||^{-s} \operatorname{sgn}^{s+1} \rtimes \mathbf{1} \rightarrow X(s,+) \oplus X(s,-) .
\end{aligned}
$$


(iii) Let $s=0$. Then there are two non-equivalent tempered irreducible representations (limits of discrete series) $X(0,+)$ (of lowest $K_{\mathbb{R}}$-type 1 ) and $X(0,-)$ (of highest $K_{\mathbb{R}}$-type -1 ) such that:

$$
\operatorname{sgn} \rtimes \mathbf{1} \simeq X(0,+) \oplus X(0,-) .
$$

(iv) All discrete series of $S L_{2}(\mathbb{R})$ are described as follows:

$$
\operatorname{Irr}\left(S L_{2}(\mathbb{R})\right)^{d i s c}=\left\{X(s,+), X(s,-) ; s \in \mathbb{Z}_{>0}\right\} .
$$

The next theorem concerns representations of $G L_{2}(\mathbb{R})$. We say that $\delta \in \operatorname{Irr}\left(G L_{2}(\mathbb{R})\right)$ is square-integrable (resp., essentially square-integrable) modulo center if it poses a globalization $\mathcal{H}_{\delta}$ (resp., a globalization $\mathcal{H}_{\delta}$ and a positive character $\eta: G L_{2}(\mathbb{R}) \rightarrow \mathbb{R}_{>0}$ ) such that $\mathcal{H}_{\delta}$ (resp., $\eta \otimes \mathcal{H}_{\delta}$ ) has a non-trivial matrix coefficient $f$ which is square-integrable modulo center: $\int_{Z \backslash G L_{2}(\mathbb{R})}|f(g)|^{2} d g<\infty$.

Theorem 2.5. Let $G(\mathbb{R})=G L_{2}(\mathbb{R})$. Put $\eta_{i}=\left.||\right|^{s_{i}}$ sgn $^{\epsilon_{i}}, s_{i} \in \mathbb{C}, \epsilon_{i} \in$ $\{0,1\}, \eta=||^{s} \operatorname{sgn}^{\epsilon}, s \in \mathbb{C}, \epsilon \in\{0,1\}$. Then we have the following:

(i) $\eta_{1} \times \eta_{2}$ is irreducible unless $\eta_{1} \eta_{2}^{-1}=||^{k} \operatorname{sgn}^{k+1}, k \in \mathbb{Z} \backslash\{0\}$. In any case, $\eta_{1} \times \eta_{2}$ and $\eta_{2} \times \eta_{1}$ have the same composition series.

(ii) $\eta||^{k / 2} \operatorname{sgn}^{k+1} \times \eta||^{-k / 2}, k>0$, has a unique irreducible subrepresentation that we denote by $\delta(\eta, k)$ and a unique irreducible quotient that we denote by $\zeta(\eta, k) . \delta(\eta, k) \simeq \delta\left(\eta^{\prime}, k^{\prime}\right)$ if and only if $\eta^{\prime} \in\{\eta$, sgn $\cdot \eta\}$ and $k=k^{\prime}$. For different pairs $(\eta, k)$ the representations $\zeta(\eta, k)$ are $m u$ tually non-isomorphic (since they are Langlands quotients). $\delta(\eta, k)$ is an essentially square-integrable modulo center representation. $\delta(\eta, k)$ is square-integrable if and only if $\eta$ is unitary. Finally, we have the following:

$$
\begin{aligned}
& \delta(\eta, k) \hookrightarrow \eta||^{k / 2} \operatorname{sgn}^{k+1} \times \eta||^{-k / 2} \rightarrow \zeta(\eta, k), \\
& \zeta(\eta, k) \hookrightarrow \eta||^{-k / 2} \times \eta||^{k / 2} \operatorname{sgn}^{k+1} \rightarrow \delta(\eta, k) .
\end{aligned}
$$

(iii) $\left.\left(\eta||^{k / 2} \operatorname{sgn}^{k+1} \times \eta||^{-k / 2}\right)\right|_{S L_{2}(\mathbb{R})} \simeq||^{k} \operatorname{sgn}^{k+1} \rtimes \mathbf{1}, k>0 . \zeta(\eta, k)$ is a finite-dimensional representation of dimension $k$, having restriction $V_{k}$ to $S L_{2}(\mathbb{R})$.

(iv) $U(1)$ is subgroup of $K_{\mathbb{R}}=O(2)$ of index two. Note that $\left[\begin{array}{ll}0 & 1 \\ 1 & 0\end{array}\right]$ acts on a character $z \rightsquigarrow z^{j}$ by sending it to $z \rightsquigarrow z^{-j}$. Thus the set of irreducible representation is described by $W_{j}:=\operatorname{Ind}_{U(1)}^{O(2)}\left(z \rightsquigarrow z^{j}\right), j \neq 0$, and trivial and determinant character (note that $W_{i} \simeq W_{j}$ if and only if $j \in\{-i, i\})$. Moreover, we have the following:

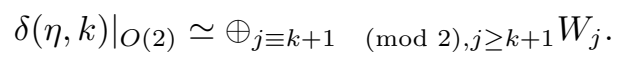


Finally, we have the following:

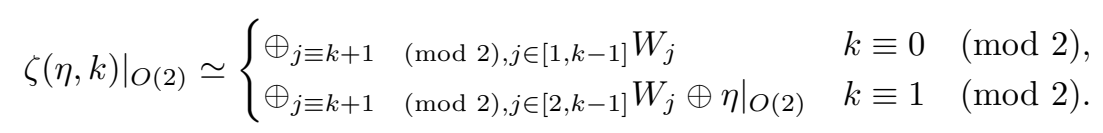

\section{Translation Functors}

In this section we describe basic construction related to translation functors following [10] (see also [27], Section 5) and prove some lemmas. We continue with the notation from the previous section.

Let $\Sigma_{1}^{+}$be some positive root system in $\Sigma$. We say that $\lambda \in \mathfrak{t}_{\mathbb{C}}^{*}$ is a $\Sigma_{1}^{+}-$ dominant weight if $\left\langle\operatorname{Re}(\lambda), \alpha^{\vee}\right\rangle \in \mathbb{R}_{\geq 0}$, for all $\alpha \in \Sigma_{1}^{+}$, and if $\left\langle\operatorname{Re}(\lambda), \alpha^{\vee}\right\rangle=0$, for all $\alpha \in \Sigma_{1}^{+}$, we require $\left\langle\operatorname{Im}(\lambda), \alpha^{\vee}\right\rangle \in \mathbb{R}_{\geq 0}$, for all $\alpha \in \Sigma_{1}^{+}$, where we take real part and imaginary part with respect to obvious real structure on $\mathfrak{t}_{\mathbb{C}}^{*}$. All $\Sigma_{1}^{+}$-dominant weights form a fundamental domain for the action of $W$ on $\mathfrak{t}_{\mathbb{C}}^{*}$. We write $D\left(\Sigma_{1}^{+}\right)$for the set of all $\Sigma_{1}^{+}$-dominant weights in $\mathfrak{t}_{\mathbb{C}}^{*}$. There is a unique $w \in W$ such that $w\left(\Sigma^{+}\right)=\Sigma_{1}^{+}$. This implies that $w\left(D\left(\Sigma^{+}\right)\right)=D\left(\Sigma_{1}^{+}\right)$.

Fixing any positive root system $\Sigma_{1}^{+}$, there is a one-to-one correspondence between dominant integral weights $\lambda \in D\left(\Sigma_{1}^{+}\right) \cap X(T)$ and algebraic finite dimensional irreducible representations of $G(\mathbb{C}), \lambda \rightsquigarrow V_{\lambda}$, characterized by that $\lambda$ is a highest weight when $V_{\lambda}$ is restricted to $T(\mathbb{C})$. Clearly, taking $\left.V_{\lambda}\right|_{G(\mathbb{R})}$ one gets a proper subset of all smooth irreducible representations of $G(\mathbb{R})$. It is characterized by the fact that $\lambda$ is highest weight of $V_{\lambda}$. We write $V^{-\lambda}$ for its dual. It is module of lowest weight $-\lambda$. This also gives parameterization of a subset of all smooth irreducible finite dimensional $G(\mathbb{R})$ representations.

Let $\mathcal{M}\left(\mathfrak{g}_{\mathbb{R}}, K_{\mathbb{R}}\right)$ be the category of finite-length admissible $\left(\mathfrak{g}_{\mathbb{R}}, K_{\mathbb{R}}\right)$ modules. For any $\lambda \in \mathfrak{t}_{\mathbb{C}}^{*}$, we define the functor $p_{\lambda}$ on $\mathcal{M}\left(\mathfrak{g}_{\mathbb{R}}, K_{\mathbb{R}}\right)$ that maps a module $X$ onto a module $p_{\lambda}(X)$, which is by the definition the largest submodule of $X$ where $z-\chi_{\lambda}(z)$ is nilpotent, for any $z \in Z\left(\mathfrak{g}_{\mathbb{C}}\right)$. Clearly, $p_{\lambda}=p_{w(\lambda)}$, for any $w \in W$. Also, this is an exact functor and $X \simeq \oplus_{\lambda} p_{\lambda}(X)$, where $\lambda$ ranges over some finite set in $\mathfrak{t}_{\mathbb{C}}^{*}$.

Now, we fix some positive root system $\Sigma_{1}^{+}$and let $\lambda \in D\left(\Sigma_{1}^{+}\right)$and $\mu \in$ $D\left(\Sigma_{1}^{+}\right) \cap X(T)$. We define two functors on $\mathcal{M}\left(\mathfrak{g}_{\mathbb{R}}, K_{\mathbb{R}}\right)$

$$
\varphi_{\lambda+\mu}^{\lambda}(X):=p_{\lambda+\mu}\left(p_{\lambda}(X) \otimes V_{\mu}\right),
$$

and

$$
\psi_{\lambda}^{\lambda+\mu}(Y):=p_{\lambda}\left(p_{\lambda+\mu}(Y) \otimes V^{-\mu}\right) .
$$

Remark 3.1. Clearly, for any $w \in W, w(\lambda) \in D\left(w\left(\Sigma_{1}^{+}\right)\right)$and $w(\mu) \in$ $D\left(w\left(\Sigma_{1}^{+}\right)\right) \cap X(T)$, and the functor $\varphi_{w(\lambda)+w(\mu)}^{w(\lambda)}\left(\right.$ resp., $\left.\psi_{w(\lambda)}^{w(\lambda)+w(\mu)}\right)$ is naturally isomorphic to $\varphi_{\lambda+\mu}^{\lambda}$ (resp., $\left.\psi_{\lambda}^{\lambda+\mu}\right)$. 
TheOREM 3.2. Let $\lambda \in D\left(\Sigma_{1}^{+}\right)$and $\mu \in D\left(\Sigma_{1}^{+}\right) \cap X(T)$ for some positive root system $\Sigma_{1}^{+}$in $\Sigma$. We write $W(\lambda+\mu)$ and $W(\lambda)$ for stabilizers in $W$ of the corresponding weights. Assume that $X, Y$ are objects of the category $\mathcal{M}\left(\mathfrak{g}_{\mathbb{R}}, K_{\mathbb{R}}\right)$. Assume that $X$ has the infinitesimal character $\lambda$ and $Y$ has the infinitesimal character $\lambda+\mu$. Then we have the following:

(i) $\varphi_{\lambda+\mu}^{\lambda}(X)=0$ implies $X=0$.

(ii) Assume that $X$ is irreducible. Then $\psi_{\lambda}^{\lambda+\mu}\left(\varphi_{\lambda+\mu}^{\lambda}(X)\right)$ has a composition series of exactly \#(W( $\lambda) / W(\lambda+\mu))$ elements. Moreover, any irreducible constituent of $\varphi_{\lambda+\mu}^{\lambda}(X)$ maps under $\psi_{\lambda}^{\lambda+\mu}$ to a module that $i s$ either zero or it has all irreducible constituents isomorphic to $X$.

(iii) If we have the equality of stabilizers $W(\lambda+\mu)=W(\lambda)$ (in $W$ ), then

$$
\begin{aligned}
\psi_{\lambda}^{\lambda+\mu}\left(\varphi_{\lambda+\mu}^{\lambda}(X)\right) & \simeq X, \\
\varphi_{\lambda+\mu}^{\lambda}\left(\psi_{\lambda}^{\lambda+\mu}(Y)\right) & \simeq Y .
\end{aligned}
$$

(iv) Assume that an module with a generalized infinitesimal character $\lambda+\mu$ has a composition series consisting of isomorphic (irreducible) pieces, say $Y$, then its image under $\psi_{\lambda}^{\lambda+\mu}$ is zero or it has a composition series composition series consisting of isomorphic (irreducible) pieces, say $X$. Moreover, if the whole module is irreducible and isomorphic to $Y$, then $Y$ appears in $\varphi_{\lambda+\mu}^{\lambda}(X)$.

(v) $\operatorname{Hom}_{\left(\mathfrak{g}_{\mathbb{R}}, K_{\mathbb{R}}\right)}\left(Y, \varphi_{\lambda+\mu}^{\lambda}(X)\right) \simeq \operatorname{Hom}_{\left(\mathfrak{g}_{\mathbb{R}}, K_{\mathbb{R}}\right.}\left(\psi_{\lambda}^{\lambda+\mu}(Y), X\right)$.

REMARK 3.3. Let $\lambda \in D\left(\Sigma_{1}^{+}\right)$and $\mu \in D\left(\Sigma_{1}^{+}\right) \cap X(T)$ for some positive root system $\Sigma_{1}^{+}$in $\Sigma$. It is easy to see that we have the following relation between stabilizers in $W: W(\lambda+\mu)=W(\lambda) \cap W(\mu)$.

We now write the effect of the action on the induced representation in the form we need later in the paper.

LEMMA 3.4. Let $\lambda, \mu \in \mathfrak{t}_{\mathbb{C}}^{*}$. We assume that there exists a positive root system $\Sigma_{1}^{+}$in $\Sigma$ such that $\lambda \in D\left(\Sigma_{1}^{+}\right)$and $\mu \in D\left(\Sigma_{1}^{+}\right) \cap X(T)$. Let $P=M N \subseteq$ $G$ be a standard parabolic subgroup corresponding to $\Theta \subset \Delta$. Assume that there exists a positive root system $\Sigma_{M, 1}^{+}$in that of $\Theta$ such that $\lambda \in D\left(\Sigma_{1}^{+}\right)_{M}$ and $\mu \in D\left(\Sigma_{1}^{+}\right)_{M} \cap X(T)$. Let $X_{M}$ be an admissible finite length $\left(\mathfrak{m}_{\mathbb{R}}, K \cap\right.$ $M(\mathbb{R}))$-module with infinitesimal character $\lambda$. Put $X=\operatorname{Ind}_{P(\mathbb{R})}^{G(\mathbb{R})}\left(X_{M}\right)$ (then $X$ also has infinitesimal character $\lambda)$. Then there is an inclusion:

$$
\operatorname{Ind}_{P(\mathbb{R})}^{G(\mathbb{R})}\left(\varphi_{\lambda+\mu}^{\lambda}\left(X_{M}\right)\right) \hookrightarrow \varphi_{\lambda+\mu}^{\lambda}(X) .
$$

Let $Y_{M}$ be an admissible finite length $\left(\mathfrak{m}_{\mathbb{R}}, K \cap M(\mathbb{R})\right)$-module with infinitesimal character $\lambda+\mu$. Put $Y=\operatorname{Ind}_{P(\mathbb{R})}^{G(\mathbb{R})}\left(Y_{M}\right)$ (then $Y$ also has infinitesimal character $\lambda+\mu)$. Then we have the following:

$$
\psi_{\lambda}^{\lambda+\mu}(Y) \simeq \operatorname{Ind}_{P(\mathbb{R})}^{G(\mathbb{R})}\left(\psi_{\lambda}^{\lambda+\mu}\left(Y_{M}\right)\right) .
$$


Proof. First, (3.4) is proved for example in [27, Corollary 5] (see also [10]). We prove (3.3). We consider the restriction

$$
\left.V_{\mu}\right|_{M(\mathbb{C})} \simeq V_{\mu}^{M} \oplus_{\mu^{\prime}<\mu} \operatorname{mult}\left(V_{\mu^{\prime}}^{M}, V_{\mu}\right) V_{\mu^{\prime}}^{M} .
$$

Here $\mu^{\prime}<\mu$ refers to the order with respect to $\Sigma_{1}^{+}$, but when we write $V_{\mu^{\prime}}^{M}$, we consider $\mu^{\prime}$ as a $\Sigma_{M, 1}^{+}$-dominant weight. Now, by Lie theorem [10], (3.5) implies that $\left.V_{\mu}\right|_{M(\mathbb{C}) N(\mathbb{C})}$ has a subrepresentation $V_{\mu}^{M}$ (where $N(\mathbb{C})$ acts trivially), and in its filtration other irreducible subquotients are of the form $V_{\mu^{\prime}}^{M}$ (where $N(\mathbb{C})$ acts trivially) each appearing with multiplicity $\operatorname{mult}\left(V_{\mu^{\prime}}^{M}, V_{\mu}\right)$. Now,

$$
X \otimes V_{\mu} \simeq \operatorname{Ind}_{P(\mathbb{R})}^{G(\mathbb{R})}\left(\left.X_{M} \otimes\left(V_{\mu}\right)\right|_{M(\mathbb{R}) N(\mathbb{R})}\right)
$$

immediately implies (3.3).

We will be more precise in the next lemma.

Lemma 3.5. Let $\lambda, \mu \in \mathfrak{t}_{\mathbb{C}}^{*}$. We assume that $\lambda \in D\left(\Sigma^{+}\right)$and $\mu \in D\left(\Sigma^{+}\right) \cap$ $X(T)$. Then we have the following:

(i) Let $\eta$ be a character of $T(\mathbb{R})$. We assume that its restriction $\nu$ to $T(\mathbb{R})^{0}$ is $W$-conjugate to $\lambda$, say $\nu=w_{0}(\lambda)$. Then we have the following:

$$
\varphi_{\lambda+\mu}^{\lambda}\left(\operatorname{Ind}_{B(\mathbb{R})}^{G(\mathbb{R})}(\eta)\right)=\sum_{w \in(W(\nu) \cap W(\mu)) \backslash W(\nu) w_{0}} \operatorname{Ind}_{B(\mathbb{R})}^{G(\mathbb{R})}(\eta \cdot w(\mu)),
$$

in $\mathcal{R}(G(\mathbb{R}))$.

(ii) Let $\eta$ be a character of $T(\mathbb{R})$. We assume that its restriction $\nu$ to $T(\mathbb{R})^{0}$ is $W$-conjugate to $\lambda+\mu$. We fix $w_{0}$ such that $w_{0}(\lambda+\mu)=\nu$. Then we have the following:

$$
\psi_{\lambda}^{\lambda+\mu}\left(\operatorname{Ind}_{B(\mathbb{R})}^{G(\mathbb{R})}(\eta)\right) \simeq \operatorname{Ind}_{B(\mathbb{R})}^{G(\mathbb{R})}\left(\eta\left(w_{0}(\mu)\right)^{-1}\right) .
$$

Proof. We prove (i). We first note that, in the case $M=T$, the decomposition in (3.5) is ordinary weight-decomposition of $V_{\mu}$. We write $\operatorname{mult}\left(\mu^{\prime}, V_{\mu}\right)$ for the dimension of weight space of $\mu^{\prime}$ in $V_{\mu}$. Hence

$$
\varphi_{\lambda+\mu}^{\lambda}\left(\operatorname{Ind}_{B(\mathbb{R})}^{G(\mathbb{R})}(\eta)\right)=\operatorname{Ind}_{B(\mathbb{R})}^{G(\mathbb{R})}(\eta \mu)+\sum_{\mu^{\prime}<\mu} \operatorname{mult}\left(\mu^{\prime}, V_{\mu}\right) p_{\lambda+\mu}\left(\operatorname{Ind}_{B(\mathbb{R})}^{G(\mathbb{R})}\left(\eta \mu^{\prime}\right)\right)
$$

where we compute in $\mathcal{R}(G(\mathbb{R}))$. The term $\operatorname{Ind}_{B(\mathbb{R})}^{G(\mathbb{R})}\left(\eta \mu^{\prime}\right)$ has infinitesimal character $\lambda+\mu$ if and only if there exists $w \in W$ such that $w^{-1}\left(\nu+\mu^{\prime}\right)=\lambda+\mu$. We write $\nu=w_{0}(\lambda)$, and we obtain the following:

$$
w^{-1} w_{0}(\lambda)+w^{-1}\left(\mu^{\prime}\right)=\lambda+\mu .
$$

This means

$$
0 \geq w^{-1}\left(\mu^{\prime}\right)-\mu=\lambda-w^{-1} w_{0}(\lambda) \geq 0 .
$$

Hence $w^{-1}\left(\mu^{\prime}\right)=\mu$ and $w^{-1} w_{0}(\lambda)=\lambda$. Thus, we see that $w \in w_{0} W(\lambda)$, $\mu^{\prime}=w(\mu)$, and $\operatorname{mult}\left(\mu^{\prime}, V_{\mu}\right)=1$. Finally, if $w, w^{\prime} \in w_{0} W(\lambda)$ satisfy $w(\mu)=$ 
$w^{\prime}\left(\mu^{\prime}\right)$, then $w^{\prime} w^{-1} \in W(\mu)$. We can write $w=w_{0} w_{1}$ and $w^{\prime}=w_{0} w_{1}^{\prime}$, where $w_{1}, w_{1}^{\prime} \in W(\lambda)$ Hence $w^{\prime} w^{-1}=w_{0}\left(w_{1}^{\prime} w_{1}^{-1}\right) w_{0}^{-1}$. But the left-hand side of the last formula is in $W(\mu)$ while the right-hand side is in $W(\nu)$. Hence $w^{\prime} w^{-1} \in W(\nu) \cap W(\mu)$. This proves the displayed formula in (i).

We prove (ii). We note that taking contragredients in (3.5), we have the following (see the last proof for the notation):

$$
\left.V^{-\mu}\right|_{T(\mathbb{C})} \simeq \oplus_{\mu^{\prime} \leq \mu} \operatorname{mult}\left(\mu^{\prime}, V_{\mu}\right)\left(V^{-\mu}\right)_{\mu^{\prime}} .
$$

Hence

$$
\psi_{\lambda}^{\lambda+\mu}\left(\operatorname{Ind}_{B(\mathbb{R})}^{G(\mathbb{R})}(\eta)\right)=\sum_{\mu^{\prime} \leq \mu} \operatorname{mult}\left(\mu^{\prime}, V_{\mu}\right) p_{\lambda}\left(\operatorname{Ind}_{B(\mathbb{R})}^{G(\mathbb{R})}\left(\eta\left(\mu^{\prime}\right)^{-1}\right)\right)
$$

in $\mathcal{R}(G(\mathbb{R}))$. Note that $\operatorname{Ind}_{B(\mathbb{R})}^{G(\mathbb{R})}\left(\eta\left(\mu^{\prime}\right)^{-1}\right)$ has infinitesimal character $\chi_{\nu-\mu^{\prime}}$. It is equal to $\chi_{\lambda}$ if there exists $w \in W$ such that $w(\lambda)=\nu-\mu^{\prime}$. Since we have fixed $w_{0}$ such that $w_{0}(\lambda+\mu)=\nu$. We have the following computation:

$$
w_{0}^{-1} w(\lambda)=w_{0}^{-1}(\nu)-w_{0}^{-1}\left(\mu^{\prime}\right)=\lambda+\mu-w_{0}^{-1}\left(\mu^{\prime}\right) .
$$

Hence

$$
0 \geq w_{0}^{-1} w(\lambda)-\lambda=\mu-w_{0}^{-1}\left(\mu^{\prime}\right) \geq 0 .
$$

Thus, $\lambda=w_{0}^{-1} w(\lambda)$ and $\mu^{\prime}=w_{0}(\mu)$. Hence $w_{0}^{-1} w \in W(\lambda)$, and now, it is easy to see that (ii) holds.

\section{Translation Functors fOR $G L_{2}(\mathbb{R})$}

In this section we prove two lemmas that we need later in the determination of the composition series of generalized and degenerate principal series of $S p(4, \mathbb{R})$.

Irreducible finite dimensional holomorphic representations of $G L_{n}(\mathbb{C})$ (or equivalently irreducible representations of $U(n))$ are parameterized by weights $\mu=\left(m_{1}, \ldots, m_{n}\right) \in \mathbb{Z}^{n}, m_{1} \geq m_{2} \geq \ldots \geq m_{n}$. We write $V_{\mu}$ for the corresponding representation.

Lemma 4.1. Let $G(\mathbb{R})=G L_{2}(\mathbb{R})$ and let $\mu=\left(m_{1}, m_{2}\right), m_{i} \in \mathbb{Z}, m_{1} \geq$ $m_{2}$. Then we have the following:

(i) Let $\eta_{i}=\operatorname{sgn}^{\epsilon_{i}}||^{s_{i}}, i=1,2$. We write $\lambda=\left(s_{1}, s_{2}\right)$ for the infinitesimal character of $\eta_{1} \times \eta_{2}$ (assumed to be dominant ${ }^{2}$ ). Then we have the following:

1. If $m_{1}>m_{2}$ and $s_{1}=s_{2}$, then we have the following exact sequence:

$$
\begin{aligned}
& \eta_{1}||^{m_{1}} \operatorname{sgn}^{m_{1}} \times \eta_{2}||^{m_{2}} \operatorname{sgn}^{m_{2}} \hookrightarrow \\
& \varphi_{\lambda+\mu}^{\lambda}\left(\eta_{1} \times \eta_{2}\right) \rightarrow \eta_{1}||^{m_{2}} \operatorname{sgn}^{m_{2}} \times \eta_{2}||^{m_{1}} \operatorname{sgn}^{m_{1}} .
\end{aligned}
$$

${ }^{2}$ i.e., $\operatorname{Re}\left(s_{1}\right)>\operatorname{Re}\left(s_{2}\right)$ or $\operatorname{Re}\left(s_{1}\right)=\operatorname{Re}\left(s_{2}\right)$ and $\operatorname{Im}\left(s_{1}\right) \geq \operatorname{Im}\left(s_{2}\right)$ (see the beginning of Section 3). 
2. If the above conditions in (1) do not hold, then we have the following:

$$
\varphi_{\lambda+\mu}^{\lambda}\left(\eta_{1} \times \eta_{2}\right) \simeq \eta_{1}||^{m_{1}} \operatorname{sgn}^{m_{1}} \times \eta_{2}||^{m_{2}} \operatorname{sgn}^{m_{2}} .
$$

(ii) Assume that $\eta=s g n^{\epsilon}||^{s}$ is a character of $\mathbb{R}^{\times}, k \in \mathbb{Z}_{>0}$. We write $\lambda=(s+k / 2, s-k / 2)$ for the infinitesimal character of $\delta(\eta, k)$ and $\zeta(\eta, k)$. Obviously, $\lambda$ is dominant. Then we have the following:

$$
\begin{aligned}
& \varphi_{\lambda+\mu}^{\lambda}(\delta(\eta, k)) \simeq \delta\left(\eta||^{\left(m_{1}+m_{2}\right) / 2} \operatorname{sgn}^{m_{2}}, k+m_{1}-m_{2}\right), \\
& \varphi_{\lambda+\mu}^{\lambda}(\zeta(\eta, k)) \simeq \zeta\left(\eta||^{\left(m_{1}+m_{2}\right) / 2} \operatorname{sgn}^{m_{2}}, k+m_{1}-m_{2}\right),
\end{aligned}
$$

Proof. (i) follows from the proof of Lemma 3.5 (i). To prove (ii), we first note the following exact sequence:

$$
\delta(\eta, k) \hookrightarrow \eta||^{k / 2} \operatorname{sgn}^{k+1} \times \eta||^{-k / 2} \rightarrow \zeta(\eta, k) .
$$

Now, we apply exact functor $\varphi_{\lambda+\mu}^{\lambda}$ to this and we obtain:

$$
\varphi_{\lambda+\mu}^{\lambda}(\delta(\eta, k)) \hookrightarrow \varphi_{\lambda+\mu}^{\lambda}\left(\eta||^{k / 2} \operatorname{sgn}^{k+1} \times \eta||^{-k / 2}\right) \rightarrow \varphi_{\lambda+\mu}^{\lambda}(\zeta(\eta, k)) .
$$

Next, (i) implies that $\varphi_{\lambda+\mu}^{\lambda}\left(\eta||^{k / 2} \operatorname{sgn}^{k+1} \times \eta||^{-k / 2}\right)$ is isomorphic to

$$
\begin{aligned}
&\left(\eta||^{\left(m_{1}+m_{2}\right) / 2} \operatorname{sgn}^{m_{2}}\right)||^{\left(k+m_{1}-m_{2}\right) / 2} \operatorname{sgn}^{k+\left(m_{1}-m_{2}\right)+1} \\
& \times\left(\eta||^{\left(m_{1}+m_{2}\right) / 2} \operatorname{sgn}^{m_{2}}\right)||^{-\left(k+m_{1}-m_{2}\right) / 2} .
\end{aligned}
$$

Also, Theorem 2.5 (ii), implies

$$
\begin{aligned}
\delta\left(\left.\eta||\right|^{\left(m_{1}+m_{2}\right) / 2} \operatorname{sgn}^{m_{2}} \eta||^{m_{2}}, k+m_{1}-m_{2}\right) & \left.\hookrightarrow \eta||^{\left(m_{1}+m_{2}\right) / 2} \operatorname{sgn}^{m_{2}}\right)||^{\left(k+m_{1}-m_{2}\right) / 2} \operatorname{sgn}^{k+\left(m_{1}-m_{2}\right)+1} \\
& \left.\times\left(\eta||^{\left(m_{1}+m_{2}\right) / 2} \operatorname{sgn}^{m_{2}}\right)||^{-\left(k+m_{1}-m_{2}\right) / 2}\right) \\
\rightarrow & \zeta\left(\eta||^{\left(m_{1}+m_{2}\right) / 2} \operatorname{sgn}^{m_{2}}, k+m_{1}-m_{2}\right) .
\end{aligned}
$$

Now, since $\varphi_{\lambda+\mu}^{\lambda}(\delta(\eta, k)) \neq 0$ and $\varphi_{\lambda+\mu}^{\lambda}(\zeta(\eta, k)) \neq 0$ (by Theorem 3.2 (i)), (4.1) and (4.2) complete the proof (ii).

Lemma 4.2. Let $G=G L_{2}(\mathbb{R})$ and let $\mu=\left(m_{1}, m_{2}\right), m_{i} \in \mathbb{Z}, m_{1} \geq m_{2}$. Then we have the following:

(i) Let $\eta_{i}=s g n^{\epsilon_{i}}||^{s_{i}}, i=1,2$. We write $\lambda+\mu=\left(s_{1}, s_{2}\right)$ for the infinitesimal character of $\eta_{1} \times \eta_{2}$ (assumed to be dominant). Assume also that $\lambda=\left(s_{1}-m_{1}, s_{2}-m_{2}\right)$ is dominant; that is $\operatorname{Re}\left(s_{1}\right)-\operatorname{Re}\left(s_{2}\right) \geq$ $m_{1}-m_{2} \geq 0$, and if $\operatorname{Re}\left(s_{1}\right)-\operatorname{Re}\left(s_{2}\right)=0$, then $\operatorname{Im}\left(s_{1}\right)-\operatorname{Im}\left(s_{2}\right) \geq 0$. Then we have the following:

$$
\psi_{\lambda}^{\lambda+\mu}\left(\eta_{1} \times \eta_{2}\right)=\eta_{1}||^{-m_{1}} \mathrm{sgn}^{-m_{1}} \times \eta_{2}||^{-m_{2}} \mathrm{sgn}^{-m_{2}} .
$$

If we assume that $\eta_{1} \times \eta_{2}$ is irreducible (that is, $\eta_{1} / \eta_{2}$ is not of the form ||$\left.^{k} \operatorname{sgn}^{k+1}, k \in \mathbb{Z}_{\neq 0}\right)$, then $\eta_{1}||^{-m_{1}} \operatorname{sgn}^{-m_{1}} \times \eta_{2}||^{-m_{2}} \operatorname{sgn}^{-m_{2}}$ is irreducible. 
(ii) Assume that $\eta=\left.s g n^{\epsilon}\right|^{s}$ is a character of $\mathbb{R}^{\times}, k \in \mathbb{Z}_{>0}$. We write $\lambda+\mu=(s+k / 2, s-k / 2)$ for the infinitesimal character of $\delta(\eta, k)$ and $\zeta(\eta, k)$. Assume that $k \geq m_{1}-m_{2} \geq 0$ so that $\lambda=\left(s+k / 2-m_{1}, s-\right.$ $\left.k / 2-m_{2}\right)$ is also dominant. Then we have the following:

$$
\begin{aligned}
& \psi_{\lambda}^{\lambda+\mu}(\delta(\eta, k)) \simeq \\
& \begin{cases}\delta\left(\eta||^{-\left(m_{1}+m_{2}\right) / 2} \operatorname{sgn}^{-m_{2}}, k-\left(m_{1}-m_{2}\right)\right) & k>m_{1}-m_{2}, \\
\eta||^{-\left(m_{1}+m_{2}\right) / 2} \operatorname{sgn}^{-m_{2}+1} \times \eta||^{-\left(m_{1}+m_{2}\right) / 2} \operatorname{sgn}^{-m_{2}} & k=m_{1}-m_{2}\end{cases} \\
& \text { and } \\
& \psi_{\lambda}^{\lambda+\mu}(\zeta(\eta, k)) \simeq \\
& \begin{cases}\zeta\left(\eta||^{-\left(m_{1}+m_{2}\right) / 2} \operatorname{sgn}^{-m_{2}}, k-\left(m_{1}-m_{2}\right)\right) & k>m_{1}-m_{2} \\
0 & k=m_{1}-m_{2} .\end{cases}
\end{aligned}
$$

Proof. (i) follows form Lemma 3.5 (ii). To prove (ii), observe that $k>m_{1}-m_{2}$, means that both $\lambda$ and $\lambda+\mu$ are regular. Therefore, we apply Theorem 3.2 (iii) to see that $\psi_{\lambda}^{\lambda+\mu}(\zeta(\eta, k)) \neq 0$ and $\psi_{\lambda}^{\lambda+\mu}(\delta(\eta, k)) \neq 0$. In this case we may proceed as in the proof of Lemma 4.1 (ii) . Finally, assume that $k=m_{1}-m_{2}$. In this case, we have the following:

$$
\begin{aligned}
& \psi_{\lambda}^{\lambda+\mu}(\left.\eta||^{k} \operatorname{sgn}^{k+1} \times \eta\right) \\
& \simeq\left(\eta||^{-\left(m_{1}+m_{2}\right) / 2} \operatorname{sgn}^{-m_{2}}\right)||^{\left(k+m_{2}-m_{1}\right) / 2} \operatorname{sgn}^{k+m_{2}-m_{1}+1} \\
& \quad \times\left(\eta||^{-\left(m_{1}+m_{2}\right) / 2} \operatorname{sgn}^{-m_{2}}\right)||^{-\left(k+m_{2}-m_{1}\right) / 2},
\end{aligned}
$$

and, therefore, an exact sequence $\left(k=m_{1}-m_{2}\right)$ :

$$
\begin{aligned}
\psi_{\lambda}^{\lambda+\mu}(\delta(\eta, k)) \hookrightarrow & \left(\eta||^{-\left(m_{1}+m_{2}\right) / 2} \operatorname{sgn}^{-m_{2}}\right)||^{\left(k+m_{2}-m_{1}\right) / 2} \operatorname{sgn}^{k+m_{2}-m_{1}+1} \\
(4.3) & \times\left(\eta||^{-\left(m_{1}+m_{2}\right) / 2} \operatorname{sgn}^{-m_{2}}\right)||^{-\left(k+m_{2}-m_{1}\right) / 2} \\
\rightarrow & \psi_{\lambda}^{\lambda+\mu}(\zeta(\eta, k)) .
\end{aligned}
$$

Since the induced representation in the middle is irreducible (see Theorem 2.5), we see that exactly one of the representations $\psi_{\lambda}^{\lambda+\mu}(\zeta(\eta, k))$ and $\psi_{\lambda}^{\lambda+\mu}(\delta(\eta, k))$ must be zero and the other one must be isomorphic to that induced representation. Since obviously $\psi_{\lambda}^{\lambda+\mu}(\zeta(\eta, k))$ is finite dimensional, we are done.

We write a corollary to the above discussion.

Corollary 4.3. Let $\eta=||^{s}$ sgn $^{\epsilon}$ be a character of $\mathbb{R}^{\times}, k \in \mathbb{Z}_{>0}$. Then we define dominant weights by $\mu=(k, 0), \lambda+\mu=(s+k / 2, s-k / 2)$, and 
$\lambda=(s-k / 2, s-k / 2)$. Then we have the following:

$$
\begin{aligned}
\psi_{\lambda}^{\lambda+\mu}\left(\eta||^{k / 2} s g n^{k+1} \times \eta||^{-k / 2}\right) & \\
& \simeq \psi_{\lambda}^{\lambda+\mu}(\delta(\eta, k)) \simeq \eta||^{-k / 2} \operatorname{sgn} \times \eta||^{-k / 2} .
\end{aligned}
$$

Moreover,

$$
X:=\varphi_{\lambda+\mu}^{\lambda}\left(\psi_{\lambda}^{\lambda+\mu}(\delta(\eta, k))\right)
$$

has the following filtration:

$$
X_{0} \subset X_{1} \subset X_{2} \subset X_{2} \subset X,
$$

where

$$
\left\{\begin{array}{l}
X_{0} \simeq \delta(\eta, k) \\
X_{1} / X_{0} \simeq \zeta(\eta, k), \\
X_{2} / X_{1} \simeq \zeta(\eta \cdot s g n, k), \\
X / X_{2} \simeq \delta(\eta, k) .
\end{array}\right.
$$

Proof. The statement (4.4) follows directly from Lemma 4.2 (ii). Next, using (4.4) and Lemma 4.1 (i), $X$ has a filtration that has $\left.\eta\right|^{k / 2} \operatorname{sgn}^{k+1} \times$ $\left.\eta\right|^{-k / 2}$ for a subrepresentation and

$$
\eta||^{-k / 2} \operatorname{sgn} \times \eta||^{k / 2} \operatorname{sgn}^{k} \simeq(\eta \cdot s g n)||^{-k / 2} \times(\eta \cdot \operatorname{sgn})||^{k / 2} \operatorname{sgn}^{k+1},
$$

since $s g n^{2}=1$. Now, we apply Theorem 2.5 to prove the claim.

\section{Principal Series of $S p(4, \mathbb{R})$}

In this section we start to investigate the composition series of principal series, generalized principal series, and degenerate principal series of $G(\mathbb{R})=$ $S p(4, \mathbb{R})$ proving two preliminary results. We start by the following lemma due to Speh [27]:

Lemma 5.1. Assume that $\eta_{i}=||^{s_{i}}$ sgn $^{\epsilon_{i}}, i=1,2$, are the characters of $\mathbb{R}^{\times}$. Then the principal series $\eta_{1} \times \eta_{2} \rtimes \mathbf{1}$ reduces if and only one of the following holds:

$$
\begin{aligned}
& s_{i} \in \mathbb{Z}, \epsilon_{i} \equiv\left(s_{i}+1\right) \quad(\bmod 2), \text { for some } i \in\{1,2\} . \\
& \eta_{1} \eta_{2}=||^{k} \text { sgn }^{k+1}, \text { for some } k \in \mathbb{Z}_{\neq 0} . \\
& \eta_{1} \eta_{2}^{-1}=||^{k} \text { sgn }{ }^{k+1}, \text { for some } k \in \mathbb{Z}_{\neq 0} .
\end{aligned}
$$

Proof. For convenience of the reader, we give a more direct proof. We remark that the reducibility conditions are invariant under the action of the Weyl group. Therefore, we may assume that $\operatorname{Re}\left(s_{1}\right) \geq \operatorname{Re}\left(s_{2}\right) \geq 0$. First, the case $\operatorname{Re}\left(s_{1}\right)=R\left(s_{2}\right)=0$ follows form the Goldberg's and Herb's work 
$([4,5])$ on $R$-groups since it is based entirely on the Harish-Chandra's theory of $R$-groups which is the same for archimedean and non-archimedean fields.

Next, we consider the case $R e\left(s_{1}\right) \geq R\left(s_{2}\right)>0$. Then $\eta_{1} \times \eta_{2} \rtimes \mathbf{1}$ is a standard representation (as defined in the statement of Theorem 2.3). We use a method of the factorization of the long-intertwining operators developed by Speh ([27]). We consider the following factorization of the long-intertwining operator:

$\eta_{1} \times \eta_{2} \rtimes \mathbf{1} \rightarrow \eta_{1} \times \eta_{2}^{-1} \rtimes \mathbf{1} \rightarrow \eta_{2}^{-1} \times \eta_{1} \rtimes \mathbf{1} \rightarrow \eta_{2}^{-1} \times \eta_{1}^{-1} \rtimes \mathbf{1} \rightarrow \eta_{1}^{-1} \times \eta_{2}^{-1} \rtimes \mathbf{1}$.

All intertwining maps in (5.1) are induced from the corresponding longintertwining operators of $G L_{2}(\mathbb{R})$ or $S L_{2}(\mathbb{R})$. Therefore, they are holomorphic. Also, the image of the composition of all intertwining operators in (5.1) is exactly $\operatorname{Lang}\left(\eta_{1} \times \eta_{2} \rtimes \mathbf{1}\right)$. Therefore, $\eta_{1} \times \eta_{2} \rtimes \mathbf{1}$ reduces if and only if $\eta_{i} \rtimes 1$ reduces (for some $i \in\{1,2\}$ ) or $\eta_{1}^{ \pm 1} \times \eta_{2}^{ \pm 1}$ reduces for some choice of signs. Now, Theorem 2.4 and Theorem 2.5 complete the proof in this case.

Finally, we consider the case $\operatorname{Re}\left(s_{1}\right)>R\left(s_{2}\right)=0$. If $\eta_{2} \rtimes \mathbf{1}$ is reducible, then the lemma follows from Theorem 2.4 (i). So, assume that $\eta_{2} \rtimes \mathbf{1}$ is irreducible. Then $\eta_{1} \times \eta_{2} \rtimes \mathbf{1}$ is a standard representation. In this case we have the following chain of intertwining operators:

$$
\eta_{1} \times \eta_{2} \rtimes \mathbf{1} \rightarrow \eta_{2} \times \eta_{1} \rtimes \mathbf{1} \rightarrow \eta_{2} \times \eta_{1}^{-1} \rtimes \mathbf{1} \rightarrow \eta_{1}^{-1} \times \eta_{2} \rtimes \mathbf{1} .
$$

Again, all intertwining operators are induced from the corresponding longintertwining operators of $G L_{2}(\mathbb{R})$ or $S L_{2}(\mathbb{R})$. Therefore, they are all holomorphic. The image of the composition of all intertwining operators in (5.2) is exactly the Langlands quotient. The lemma follows as in the previous case.

Corollary 5.2. Assume that $\eta, \eta_{1}$ and $\eta_{2}$ are unitary characters of $\mathbb{R}^{\times}$. Then $\eta_{1} \times \eta_{2} \rtimes \mathbf{1}$ is irreducible unless $\eta_{1}=$ sgn or $\eta_{2}=$ sgn. Next, the induced representations $\eta \rtimes X(0, \pm)$ are irreducible, and we have the following:

$$
\operatorname{sgn} \times \eta \rtimes \mathbf{1} \simeq \eta \times \operatorname{sgn} \rtimes \mathbf{1} \simeq \eta \rtimes X(0,+) \oplus \eta \rtimes X(0,-) .
$$

Proof. This follows from Lemma 5.1 and Theorem 2.4.

\section{On the RESTRICTION TO $K_{\mathbb{R}}$}

Again, we let $G(\mathbb{R})=S p(4, \mathbb{R})$. We note that $K_{\mathbb{R}} \simeq U(2)$. In this section we compute the restriction to $K_{\mathbb{R}}=U(2)$ of various induced representations. We use the notation $V_{\left(k_{1}, k_{2}\right)}$ introduced at the beginning of Section 5 . Restricting that representation to the standard torus in $S L_{2}(\mathbb{C})$ (or that of $S U(2)$ ), we find the following weights: $k_{1}-k_{2}, k_{1}-k_{2}-2, \cdots,-\left(k_{1}-k_{2}\right)$. Since the center of $G L_{2}(\mathbb{C})$ acts on the each of the corresponding weight spaces as the same scalar $k_{1}+k_{2}$, we see that all weight spaces of $V_{\left(k_{1}, k_{2}\right)}$ (for the standard 
torus of $G L_{2}(\mathbb{C})$ or of $\left.U(2)\right)$ are one dimensional and they are $\left(k_{1}-i, k_{2}+i\right)$, $0 \leq i \leq k_{1}-k_{2}$. Now, we are ready to compute the restrictions:

Lemma 6.1. Assume that $\eta=||^{s} \operatorname{sgn}^{\epsilon}, \eta_{i}=||^{s_{i}} \operatorname{sgn}^{\epsilon_{i}}\left(\epsilon, \epsilon_{i} \in\{0,1\}\right.$, $\left.s, s_{i} \in \mathbb{C}\right), i=1,2$, are characters of $\mathbb{R}^{\times}, p \in \mathbb{Z}_{\geq 0}$ and $k \in \mathbb{Z}_{>0}$. Then we have the following:

$$
\begin{aligned}
& \left.\quad\left(\eta_{1} \times \eta_{2} \rtimes \mathbf{1}\right)\right|_{U(2)} \simeq \\
& \oplus_{k_{1}+\epsilon_{1} \equiv k_{2}+\epsilon_{2}}(\bmod 2) \#\left\{i ; 0 \leq i \leq k_{1}-k_{2}, i \equiv k_{1}+\epsilon_{1} \quad(\bmod 2)\right\} V_{\left(k_{1}, k_{2}\right)} .
\end{aligned}
$$

Let $w \in\{ \pm\}$. Let $J(w)$ be the set of all $j$ such that $j \equiv p+1(\bmod 2)$ and $j \geq p+1(w=+)$ or $j \leq-p-1(w=-)$. Then we have the following:

$\left.(\eta \rtimes X(p, w))\right|_{U(2)} \simeq \oplus_{p \equiv k_{1}+k_{2}+\epsilon+1}(\bmod 2) \#\left\{j \in J(w) ; k_{2} \leq j \leq k_{1}\right\} V_{\left(k_{1}, k_{2}\right)}$.

Assume $p>0$. Then we have the following:

$$
\begin{aligned}
& \left.\left(\eta \rtimes V_{p}\right)\right|_{U(2)} \simeq \\
& \oplus_{p \equiv k_{1}+k_{2}+\epsilon+1(\bmod 2)} \#\left\{j ; j \equiv p+1(\bmod 2), j \in[-p+1, p-1] \cap\left[k_{2}, k_{1}\right]\right\} V_{\left(k_{1}, k_{2}\right)} .
\end{aligned}
$$

Next, we have the following:

$\left.(\delta(\eta, k) \rtimes \mathbf{1})\right|_{U(2)} \simeq \oplus_{k_{1}+k_{2} \equiv k+1(\bmod 2), k_{1}-k_{2}-k-1 \geq 0} \frac{\left(k_{1}-k_{2}-k+1\right)}{2} V_{\left(k_{1}, k_{2}\right)}$.

Proof. We remind the reader that $T$ stands for the standard torus of $S p(4)$. Then $T(\mathbb{R}) \cap K_{\mathbb{R}}=\{ \pm 1\} \times\{ \pm 1\}$. In fact, if we use standard embedding $U(1) \times U(1) \hookrightarrow U(2)$, then $T(\mathbb{R}) \cap(U(1) \times U(1))=\{ \pm 1\} \times\{ \pm 1\}$.

Now, we prove the first displayed formula. Obviously, we have

$$
\left.\left(\eta_{1} \times \eta_{2} \rtimes \mathbf{1}\right)\right|_{U(2)} \simeq \operatorname{Ind}_{T(\mathbb{R}) \cap(U(1) \times U(1))}^{U(2)}\left(\operatorname{sign}^{\epsilon_{1}} \otimes \operatorname{sign}^{\epsilon_{2}}\right) .
$$

Now, by Frobenius reciprocity the multiplicity of $V_{\left(k_{1}, k_{2}\right)}$ in $\left.\left(\eta_{1} \times \eta_{2} \rtimes \mathbf{1}\right)\right|_{U(2)}$ is equal to the number of weights $\left(k_{1}-i, k_{2}+i\right), 0 \leq i \leq k_{1}-k_{2}$ such that $(-1)^{k_{1}-i}=(-1)^{\epsilon_{1}}$ and $(-1)^{k_{2}+i}=(-1)^{\epsilon_{2}}$. The formula follows.

Let $w \in\{ \pm\}$, then we have the following:

$$
\begin{aligned}
& \left.(\eta \rtimes X(p, w))\right|_{U(2)} \simeq \\
& \operatorname{Ind}_{\{ \pm 1\} \times U(1)}^{U(2)}\left(\left.s g n^{\epsilon} \otimes X(p, w)\right|_{U(1)}\right) \simeq \oplus_{j} \operatorname{Ind}_{\{ \pm 1\} \times U(1)}^{U(2)}\left(\operatorname{sgn}^{\epsilon} \otimes\left(z \rightsquigarrow z^{j}\right)\right),
\end{aligned}
$$

where the sum runs over all $j \equiv p+1(\bmod 2)$ and $j \geq p+1(w=+)$ or $j \leq-p-1(w=-)$. Again, we use the Frobenius reciprocity to conclude that $V_{\left(k_{1}, k_{2}\right)}$ appears in $\operatorname{Ind}_{\{ \pm 1\} \times U(1)}^{U(2)}\left(\operatorname{sgn}^{\epsilon} \otimes\left(z \rightsquigarrow z^{j}\right)\right)$ if and only if there exists $i$, $0 \leq i \leq k_{1}-k_{2}$, such that $(-1)^{k_{1}-i}=(-1)^{\epsilon}$ and $k_{2}+i=j$. This is equivalent with $k_{2} \leq j \leq k_{1}, j \equiv k_{1}+k_{2}+\epsilon(\bmod 2)$. Note that $j \equiv k_{1}+k_{2}+\epsilon(\bmod 2)$ means $p \equiv k_{1}+k_{2}+\epsilon+1(\bmod 2)$. This proves the second displayed formula. 
The third displayed formula is proved similarly. Next, we have the following formula:

$$
\begin{aligned}
& \left.(\delta(\eta, k) \rtimes \mathbf{1})\right|_{U(2)} \simeq \operatorname{Ind}_{O(2)}^{U(2)}\left(\left.\delta(\eta, k)\right|_{O(2)}\right) \\
& \simeq \oplus_{j \geq k+1, j \equiv k+1 \quad(\bmod 2)} \operatorname{Ind}_{O(2)}^{U(2)}\left(W_{j}\right) \\
& \simeq \oplus_{j \geq k+1, j \equiv k+1 \quad(\bmod 2)} \operatorname{Ind}_{U(1)}^{U(2)}\left(z \rightsquigarrow z^{j}\right) \text {. }
\end{aligned}
$$

Here, $U(1)$ is embedded in the standard torus $U(1) \times U(1)$ of $U(2)$ as follows $z \rightsquigarrow\left(z, z^{-1}\right)$. Next, again by the Frobenius reciprocity, we see that the multiplicity of $V_{\left(k_{1}, k_{2}\right)}$ in $\operatorname{Ind}_{O(2)}^{U(2)}\left(\left.\delta(\eta, k)\right|_{O(2)}\right)$ is equal to the number of $j \geq$ $k+1, j \equiv k+1(\bmod 2)$ such that $k_{1}-k_{2}-2 i=j$ for some $0 \leq i \leq k_{1}-k_{2}$. This is equivalent to $k_{1}+k_{2} \equiv k+1(\bmod 2)$ and $i \in\left[0,\left(k_{1}-k_{2}-k-1\right) / 2\right]$.

\section{Poles of normalized intertwining operators}

In this section we prove some technical results related to intertwining operators which are used later for the determination of the composition series of generalized and degenerate principal series.

Lemma 7.1. Assume that $\eta=||^{s} \operatorname{sgn}^{\epsilon}$ is a character of $\mathbb{R}^{\times}$. Then the contragredient representations satisfy the following:

$$
\widetilde{\delta(\eta, k)} \simeq \delta\left(\eta^{-1}, k\right), \quad \widetilde{\zeta(\eta, k)} \simeq \zeta\left(\eta^{-1} s g n^{k+1}, k\right) .
$$

Proof. We remind the reader that $k>0$. First, by the definition (see Theorem 2.5), we have the following:

$$
\delta(\eta, k) \hookrightarrow \eta||^{k / 2} \operatorname{sgn}^{k+1} \times \eta||^{-k / 2} \rightarrow \zeta(\eta, k),
$$

and, then taking the contragredients, we obtain:

$$
\widetilde{\zeta(\eta, k)} \hookrightarrow \eta^{-1}||^{-k / 2} \operatorname{sgn}^{k+1} \times \eta^{-1}||^{k / 2} \rightarrow \widetilde{\delta(\eta, k)} .
$$

Combining this with the fact

$$
\begin{aligned}
\zeta\left(\left(\eta^{-1} \operatorname{sgn}^{k+1}\right), k\right) & \hookrightarrow\left(\eta^{-1} \operatorname{sgn}^{k+1}\right)||^{-k / 2} \times\left(\eta^{-1} \operatorname{sgn}^{k+1}\right)||^{k / 2} \operatorname{sgn}^{k+1} \\
& \rightarrow \delta\left(\left(\eta^{-1} s g n^{k+1}\right), k\right),
\end{aligned}
$$

we obtain

$$
\widetilde{\zeta(\eta, k)} \simeq \zeta\left(\eta^{-1} \operatorname{sgn}^{k+1}, k\right), \quad \widetilde{\delta(\eta, k)} \simeq \delta\left(\left(\eta^{-1} s g n^{k+1}\right), k\right) .
$$

Combining this with Theorem 2.5 (ii), we obtain $\widetilde{\delta(\eta, k)} \simeq \delta\left(\left(\eta^{-1} s g n^{k+2}\right), k\right)$. This proves the lemma.

Now, we fix some notation. Let $P=M N \subset G$ be the (standard) Siegel parabolic subgroup of $S p(4, \mathbb{R})$. We write $w_{0}$ for the representative in $G(\mathbb{R})=$ $S p(4, \mathbb{R})$ of the non-trivial element of the Weyl group $W(M) \simeq N_{G}(M) / M$. It 
acts on $M$ by conjugation $m \rightsquigarrow w_{0} m w_{0}^{-1}$. Under $M(\mathbb{R}) \simeq G L_{2}(\mathbb{R})$, the action of $w_{0}$ is given by $g \rightsquigarrow\left(g^{t}\right)^{-1}$, where $g^{t}$ is the transpose matrix of $g$. This automorphism acts on irreducible $\left(\mathfrak{g l}_{2}(\mathbb{R}), O(2)\right)$-modules in a obvious way. In fact, it transforms $\left(\mathfrak{g l}_{2}(\mathbb{R}), O(2)\right)$-module to its contragredient module as can be easily seen comparing their characters on the set of regular semisimple elements.

Assume that $\eta=||^{s} \operatorname{sgn}^{\epsilon}(s \in \mathbb{C}, \epsilon \in\{0,1\})$. We remind the reader that we write $\delta(\eta, k) \rtimes \mathbf{1}$ for $\operatorname{Ind}_{P(\mathbb{R})}^{S p(4, \mathbb{R})}(\delta(\eta, k))$.

If $\operatorname{Re}(s)>0$, then the long-intertwining operator $f \rightsquigarrow \int_{N(\mathbb{R})} f\left(w_{0}^{-1} n g\right) d n$, is a non-zero intertwining map $\delta(\eta, k) \rtimes \mathbf{1} \rightarrow \delta\left(\eta^{-1}, k\right) \rtimes \mathbf{1}$ having the image isomorphic $\operatorname{Lang}(\delta(\eta, k) \rtimes \mathbf{1})$.

We remark that a well-known result of Knapp-Zuckerman ([10]) implies that $\operatorname{Lang}(\delta(\eta, k) \rtimes \mathbf{1})$ is Hermitian if and only if $s \in \mathbb{R}$.

Finally, in $\mathcal{R}(S p(4, \mathbb{R}))$, the semisimplifications are related by $\delta(\eta, k) \rtimes \mathbf{1}=$ $\delta\left(\eta^{-1}, k\right) \rtimes \mathbf{1}$. In more detail, for generic value of $s$, the long-intertwining operator is an isomorphism, and then we use fact that character $\operatorname{tr}((\delta(\eta, k) \rtimes$ $\mathbf{1})(f))$ of $\delta(\eta, k) \rtimes \mathbf{1}$, applied to a test function $f \in C_{c}^{\infty}(S p(4, \mathbb{R}))$, is analytic in $s$.

Now, we will analyze the long-intertwining operator $\delta(\eta, k) \rtimes \mathbf{1} \rightarrow$ $\delta\left(\eta^{-1}, k\right) \rtimes 1$. Put $\eta_{u}=||^{\operatorname{Im}(s)} \operatorname{sign}^{\epsilon}$. Since $\delta\left(\eta_{u}, k\right) \hookrightarrow \eta_{u}||^{k / 2} \operatorname{sgn}^{k+1} \times$ $\eta_{u}||^{-k / 2}$, we obtain $i:|\operatorname{det}|{ }^{t} \delta\left(\eta_{u}, k\right) \rtimes \mathbf{1} \hookrightarrow \eta_{u}||^{t+k / 2} \operatorname{sgn}^{k+1} \times \eta_{u}||^{t-k / 2}$ which does not depend on $t:=\operatorname{Re}(s)$. Similarly, we have an embedding $j:|\operatorname{det}|^{-t} \delta\left(\eta_{u}^{-1}, k\right) \rtimes \mathbf{1} \hookrightarrow \eta_{u}^{-1}||^{-t+k / 2} \times \eta_{u}^{-1}||^{-t-k / 2} \operatorname{sgn}^{k+1} \rtimes \mathbf{1}$ which does not depend on $t=R e(s)$.

LEMmA 7.2. Let $t=R e(s)>0$. Then we have the following decomposition of the long intertwining operator $A(t):|\operatorname{det}|^{t} \delta\left(\eta_{u}, k\right) \rtimes \mathbf{1} \rightarrow$ $|\operatorname{det}|^{-t} \delta\left(\eta_{u}^{-1}, k\right) \rtimes \mathbf{1}$ into integral-rank one intertwining operators:

$$
\begin{array}{r}
|\operatorname{det}|{ }^{t} \delta\left(\eta_{u}, k\right) \rtimes \mathbf{1} \longrightarrow \begin{array}{r}
\eta_{u}||^{t+k / 2} \operatorname{sgn}^{k+1} \times \eta_{u}||^{t-k / 2} \rtimes \mathbf{1} \\
A_{1}(t) \downarrow \\
\eta_{u}||^{t+k / 2} \operatorname{sgn}^{k+1} \times \eta_{u}^{-1}||^{-t+k / 2} \rtimes \mathbf{1} \\
A_{2}(t) \downarrow \\
\eta_{u}^{-1}||^{-t+k / 2} \times \eta_{u}||^{t+k / 2} \operatorname{sgn}^{k+1} \rtimes \mathbf{1} \\
A_{3}(t) \downarrow
\end{array} \\
|\operatorname{det}|^{-t} \delta\left(\eta_{u}^{-1}, k\right) \rtimes \mathbf{1} \stackrel{j}{\longrightarrow} \eta_{u}^{-1}||^{-t+k / 2} \times \eta_{u}^{-1}||^{-t-k / 2} \operatorname{sgn}^{k+1} \rtimes \mathbf{1},
\end{array}
$$

where $A_{i}(t), i=1,2,3$, are induced (integral) long-intertwining operators. Moreover, we have the following: 
(i) $A_{1}(t)$ has a simple pole if there exists $p \in \mathbb{Z}_{\geq \epsilon}$ such that $s=t=$ $k / 2-p, p \equiv \epsilon(\bmod 2)$. Otherwise it is holomorphic. The intertwining operators $A_{i}(t), i=2,3$, are holomorphic for $t>0$.

(ii) Assume that $A_{1}(t)$ is holomorphic. Then it is an isomorphism unless $s=t, t-k / 2 \in \mathbb{Z}_{\neq 0}$ and $t-k / 2 \equiv \epsilon+1(\bmod 2)$. Moreover, if $t>k / 2$, then

$\operatorname{ker} A_{1}(t) \simeq \eta_{u}||^{t+k / 2} \operatorname{sgn}^{k+1} \rtimes(X(t-k / 2,+) \oplus X(t-k / 2,-))$,

$\operatorname{Im} A_{1}(t) \simeq \eta_{u}||^{t+k / 2} \operatorname{sgn}^{k+1} \rtimes V_{t-k / 2}$.

Furthermore, if $t<k / 2$, then

$\operatorname{ker} A_{1}(t) \simeq \eta_{u}||^{t+k / 2} \operatorname{sgn}^{k+1} \rtimes V_{k / 2-t}$,

$\operatorname{Im} A_{1}(t) \simeq \eta_{u}||^{t+k / 2} \operatorname{sgn}^{k+1} \rtimes(X(k / 2-t,+) \oplus X(k / 2-t,-))$.

Finally, if $s=t=k / 2$ and $\epsilon \equiv 1(\bmod 2)$, then $A_{1}(t)$ acts as a (different) multiple of identity on any of the two components of

$\eta_{u}||^{t+k / 2} \operatorname{sgn}^{k+1} \times \operatorname{sgn} \rtimes \mathbf{1} \simeq$

$$
\eta_{u}||^{t+k / 2} \operatorname{sgn}^{k+1} \rtimes X(0,+) \oplus \eta_{u}||^{t+k / 2} \operatorname{sgn}^{k+1} \rtimes X(0,-) .
$$

(iii) $A_{2}(t)$ is an isomorphism unless $s=t$ and $t-k / 2 \in \mathbb{Z}$. In this case we have the following:

$$
\begin{aligned}
& \operatorname{ker} A_{2}(t) \simeq \delta\left(||^{k / 2} \operatorname{sgn}^{\epsilon}, 2 t\right) \rtimes \mathbf{1}, \\
& \operatorname{Im} A_{2}(t) \simeq \zeta\left(||^{k / 2} \operatorname{sgn}^{\epsilon}, 2 t\right) \rtimes \mathbf{1} .
\end{aligned}
$$

(iv) $A_{3}(t)$ is an isomorphism unless $s=t, t-k / 2 \in \mathbb{Z}$ and $t-k / 2 \equiv \epsilon$ $(\bmod 2)$. In this case we have the following:

$$
\begin{aligned}
& \operatorname{ker} A_{3}(t) \simeq s g n^{\epsilon}||^{-t+k / 2} \rtimes(X(t+k / 2,+) \oplus X(t+k / 2,-)), \\
& \operatorname{Im} A_{3}(t) \simeq s g n^{\epsilon}||^{-t+k / 2} \rtimes V_{t+k / 2} .
\end{aligned}
$$

Proof. We remind the reader that we assume $t>0$. First, the commutative diagram in the lemma follows from the analytic continuation of intertwining operators [10]. Also, the long-intertwining operator $A_{2}(t)$, being induced from the long-intertwining operator of $G L_{2}(\mathbb{R})$ (and since $t+k / 2>t-k / 2$ ), is holomorphic and non-zero. Similarly, $t+k / 2>0$, implies that is longintertwining operator $A_{3}(t)$ for $S L_{2}(\mathbb{R})$ holomorphic and non-zero. Next, all statements except the one about the poles of $A_{1}(t)$ in (i) and (ii) follow from Theorem 2.4.

Now, we compute the poles of $A_{1}(t)$. We normalize the intertwining operator $A_{1}(t)$ as proposed by Langlands $([2])$. The normalization factor is given by

$$
r(t)=\pi^{-1 / 2}(\sqrt{-1})^{-\epsilon} \cdot \frac{\Gamma((1+s-k / 2+\epsilon) / 2)}{\Gamma((s-k / 2+\epsilon) / 2)} .
$$


We let

$$
R_{1}(t)=r(t) A_{1}(t)
$$

where the number $\pi$ is a usual given by $\pi=3.14 \ldots$, and $\Gamma$ is the usual gamma function. We analyze poles and zeros of $A_{1}(t)$ and $R_{1}(t)$ in the next few easy steps.

First, we remind the reader that $\Gamma$-function has no zeros and it has simple poles for each element of $\mathbb{Z}_{\leq 0}$ (and only for them). This implies $\Gamma((s-k / 2+$ $\epsilon) / 2)=\infty$ is equivalent to $s-k / 2+\epsilon \in 2 \mathbb{Z}_{\leq 0}$. This is equivalent to the following:

$$
\text { there exists } p \in \mathbb{Z}_{\geq \epsilon} \text { such that } s=t=k / 2-p, p \equiv \epsilon \quad(\bmod 2) .
$$

Similarly, $\Gamma((1+s-k / 2+\epsilon) / 2)=\infty$ is equivalent to $1+s-k / 2+\epsilon \in 2 \mathbb{Z}_{\leq 0}$. Hence, this is equivalent to the following:

$$
\text { there exists } p \in \mathbb{Z}_{\geq \epsilon+1} \text { such that } s=t=k / 2-p, p \equiv \epsilon+1 \quad(\bmod 2) .
$$

It is clear that the (simple) zeros of $r(t)$ are given by (7.4) and the (simple) poles by (7.5). Next, $A_{1}(t)$ and $R_{1}(t)$ are holomorphic and non-zero for $t>k / 2$. Further, $R_{1}(t)$ is holomorphic and non-zero for $t=k / 2$, by usual property of normalized intertwining operators. Next, by the theory of $R-$ groups, $A_{1}(t)$ has a simple pole at $t=k / 2$ if and only if $\eta_{u} \rtimes \mathbf{1}$ is irreducible and $\eta_{u}^{2}=1$. This is equivalent to $\operatorname{Im}(s)=0$ and $\epsilon=0$. Next, we show that $R_{1}(t)$ is never zero. In more detail, we put $R_{1}\left(t, \eta_{u}\right)=R_{1}(t)$ and we have that the composition of intertwining operators

$$
\eta_{u}||^{t-k / 2} \rtimes \mathbf{1} \stackrel{R_{1}\left(t, \eta_{u}\right)}{\longrightarrow} \eta_{u}^{-1}||^{-t+k / 2} \rtimes \mathbf{1} \stackrel{R_{1}\left(k-t, \eta_{u}^{-1}\right)}{\longrightarrow} \eta_{u}||^{t-k / 2} \rtimes \mathbf{1}
$$

is identity (by the usual property of the normalized intertwining operators). If $t \geq k / 2$, then $R_{1}\left(t, \eta_{u}\right)$ is non-zero. If $t<k / 2$, then $k-t>k / 2$ and $R_{1}\left(k-t, \eta_{u}^{-1}\right)$ is non-zero and holomorphic. This implies that $R_{1}(t)$ is never zero. Hence we see that the poles of $R_{1}(t)$ can exist only for $t<k / 2$. Now, (7.6) immediately shows that $R_{1}(t)$ has a pole if and only if $\eta_{u}||^{t-k / 2} \rtimes \mathbf{1}$ reduces and $t<k / 2$. Applying Theorem 2.4, for $t<k / 2, \eta_{u}||^{t-k / 2} \rtimes \mathbf{1}$ reduces if and only if

there exists $p \in \mathbb{Z}_{>0}$ such that $s=t=k / 2-p, \epsilon \equiv p+1 \quad(\bmod 2)$.

This is equivalent to (7.5). Finally, since $R_{1}(t)$ is never zero and the poles of $R_{1}(t)$ are all simple (which is well-known and follows for example from the computation of the local coefficients given in [25]), we obtain (i) from (7.3). To prove (ii), assume that $A_{1}(t)$ is holomorphic. Then clearly $A_{1}(t)$ is an isomorphism if $\eta_{u}||^{t-k / 2} \rtimes \mathbf{1}$ is irreducible. By Theorem 2.4, this happens exactly when $s=t, t-k / 2 \in \mathbb{Z}$ and $t-k / 2 \equiv \epsilon+1(\bmod 2)$. Now, if $t-k / 2 \neq 0$, then (ii) follows from Theorem 2.4. If $t=k / 2$, then $A_{1}(t)$ acts 
as a (different) multiple of identity on any of the two irreducible components of $\operatorname{sgn} \rtimes \mathbf{1} \simeq X(0,+) \oplus X(0,-)$, by the theory of $R$-groups.

Now, we consider the analogue of the previous lemma for other standard (non-Siegel) parabolic subgroup of $S p(4, \mathbb{R})$. Again, we fix some notation first. Let $P=M N \subset G$ be that maximal parabolic subgroup. We have $M(\mathbb{R}) \simeq \mathbb{R}^{\times} \times S L_{2}(\mathbb{R})$. We write $w_{0}$ for the representative in $G(\mathbb{R})$ of nontrivial element of Weyl group $W(M) \simeq N_{G}(M) / M$. It acts on $M$ by the conjugation $m \rightsquigarrow w_{0} m w_{0}^{-1}$, than under $M(\mathbb{R}) \simeq \mathbb{R}^{\times} \times S L_{2}(\mathbb{R})$ is given by $(h, g) \in \mathbb{R}^{\times} \times S L_{2}(\mathbb{R}) \rightsquigarrow\left(h^{-1}, g\right)$.

Assume that $\eta=||^{s} \operatorname{sgn}^{\epsilon}(s \in \mathbb{C}, \epsilon \in\{0,1\})$. Let $p \in \mathbb{Z}_{\geq 0}$ and let $w \in\{ \pm\}$. We remind the reader that we write $\eta \rtimes X(p, w)$ for $\operatorname{Ind}_{P(\mathbb{R})}^{S p(4, \mathbb{R})}(\eta \rtimes$ $X(p, w))$.

If $\operatorname{Re}(s)>0$, then the long-intertwining operator $f \rightsquigarrow \int_{N(\mathbb{R})} f\left(w_{0}^{-1} n g\right) d n$, is a non-zero intertwining operator $\eta \rtimes X(p, w) \rightarrow \eta^{-1} \rtimes X(p, w)$ having the image isomorphic $\operatorname{Lang}(\eta \rtimes X(p, w))$.

We remark that a well-known result of Knapp-Zuckerman ([10]) implies that $\operatorname{Lang}(\eta \rtimes X(p, w))$ is Hermitian if and only if $s \in \mathbb{R}$.

Finally, in $\mathcal{R}(S p(4, \mathbb{R}))$, the semisimplifications are related by $\eta \rtimes$ $X(p, w)=\eta^{-1} \rtimes X(p, w)$.

Now, we will analyze the long-intertwining operator. Put $\eta_{u}=$ | ${ }^{\operatorname{Im}(s)} \operatorname{sign}^{\epsilon}$. We have the embeddings

$$
\begin{aligned}
& i:\left.\left|{ }^{t} \eta_{u} \rtimes X(p, w) \hookrightarrow\right|\left|{ }^{t} \eta_{u} \times\right|\right|^{p} \operatorname{sgn}^{p+1} \rtimes \mathbf{1}, \\
& j:||^{-t} \eta_{u}^{-1} \rtimes X(p, w) \hookrightarrow||^{-t} \eta_{u}^{-1} \times||^{p} \operatorname{sgn}^{p+1} \rtimes \mathbf{1},
\end{aligned}
$$

which do not depend on $t:=\operatorname{Re}(s)$.

Lemma 7.3. Let $p \in \mathbb{Z}_{\geq 0}$ and let $t=\operatorname{Re}(s)>0$. Then we have the following decomposition of the long intertwining operator $B(t)$ into integralrank one intertwining operators:

$$
\begin{aligned}
& ||{ }^{t} \eta_{u} \rtimes X(p,+) \oplus||{ }^{t} \eta_{u} \rtimes X(p,-) \quad \stackrel{i}{\longrightarrow}||{ }^{t} \eta_{u} \times||{ }^{p} \operatorname{sgn}^{p+1} \rtimes \mathbf{1} \\
& B_{1}(t) \downarrow \\
& ||^{p} \operatorname{sgn}^{p+1} \times||{ }^{t} \eta_{u} \rtimes \mathbf{1} \\
& B(t) \downarrow \quad B_{2}(t) \downarrow \\
& \left.\left|{ }^{p} \operatorname{sgn}^{p+1} \times\right|\right|^{-t} \eta_{u}^{-1} \rtimes \mathbf{1} \\
& B_{3}(t) \downarrow \\
& ||^{-t} \eta_{u}^{-1} \rtimes X(p,+) \oplus||^{-t} \eta_{u}^{-1} \rtimes X(p,-) \stackrel{j}{\longrightarrow}||^{-t} \eta_{u}^{-1} \times||{ }^{p} \operatorname{sgn}^{p+1} \rtimes \mathbf{1},
\end{aligned}
$$


where $B_{i}(t), i=1,2,3$, are induced long-intertwining operators. Moreover, we have the following:

(i) The intertwining operators $B_{i}(t), i=2,3$, are holomorphic for $t>0$. $B_{1}(t)$ has a simple pole if and only if $s=t \in \mathbb{Z}, t \leq p, t \equiv \epsilon+1$ $(\bmod 2)$.

(ii) Assume that $B_{1}(t)$ is holomorphic. Then $B_{1}(t)$ is not an isomorphism if and only if $s=t \in \mathbb{Z}, t \neq p, t \equiv \epsilon(\bmod 2)$. Further, if $t>p$, then

$$
\begin{aligned}
& \operatorname{ker} B_{1}(t) \simeq \delta\left(||^{(t+p) / 2} s g n^{p+1}, t-p\right) \rtimes \mathbf{1}, \\
& \operatorname{Im} B_{1}(t) \simeq \zeta\left(||^{(t+p) / 2} s g n^{p+1}, t-p\right) \rtimes \mathbf{1} .
\end{aligned}
$$

Next, if $p>t$, then

$$
\begin{aligned}
& \operatorname{ker} B_{1}(t) \simeq \zeta\left(||^{(t+p) / 2} \operatorname{sgn}^{t}, p-t\right) \rtimes \mathbf{1}, \\
& \operatorname{Im} B_{1}(t) \simeq \delta\left(||^{(t+p) / 2} \operatorname{sgn}^{t}, p-t\right) \rtimes \mathbf{1} .
\end{aligned}
$$

(iii) $B_{2}(t)$ is not an isomorphism if and only if $s=t \in \mathbb{Z}, t \equiv \epsilon+1(\bmod 2)$. In that case we have the following:

$$
\begin{aligned}
& \operatorname{ker} B_{2}(t) \simeq||^{p} \operatorname{sgn}^{p+1} \rtimes(X(t,+) \oplus X(t,-)), \\
& \operatorname{Im} B_{2}(t) \simeq||^{p} \operatorname{sgn}^{p+1} \rtimes V_{t} .
\end{aligned}
$$

(iv) $B_{3}(t)$ is not an isomorphism if and only if $s=t \in \mathbb{Z}$, and $t \equiv \epsilon$ $(\bmod 2)$. In that case we have the following:

$$
\begin{aligned}
& \operatorname{ker} B_{3}(t) \simeq \delta\left(||^{(p-t) / 2} \operatorname{sgn}^{t}, p+t\right) \rtimes \mathbf{1}, \\
& \operatorname{Im} B_{3}(t) \simeq \zeta\left(||^{(p-t) / 2} \operatorname{sgn}^{t}, p+t\right) \rtimes \mathbf{1} .
\end{aligned}
$$

Proof. The parts (ii), (iii) and (iv) follow from Theorems 2.4 and 2.5. The first part of (i) is obvious. Now, we compute the poles of $B_{1}(t)$. Again we normalize the intertwining operator as proposed by Langlands:

$$
r_{1}(t)=\pi^{-1 / 2}(\sqrt{-1})^{-\epsilon^{\prime}} \cdot \frac{\Gamma\left(\left(1+s-p+\epsilon^{\prime}\right) / 2\right)}{\Gamma\left(\left(s-p+\epsilon^{\prime}\right) / 2\right)}
$$

and

$$
R_{1}(t)=r_{1}(t) B_{1}(t),
$$

where $\epsilon^{\prime} \in\{0,1\}$ is defined by $\epsilon^{\prime} \equiv \epsilon+p+1(\bmod 2)$. Now, as in the previous lemma we see that $R_{1}(t)$ has simple poles if and only if $t-p<0$ and ||${ }^{t} \eta_{u} \times||^{p} \operatorname{sgn}^{p+1}$ reduces. Therefore, $R_{1}(t)$ has a simple pole if and only if $t-p \in \mathbb{Z}_{<0}, s=t$, and $t-p+1 \equiv \epsilon+p+1(\bmod 2)$. The last condition reduces to $t \equiv \epsilon(\bmod 2)$. Finally, $R_{1}(t)$ has a simple pole if and only if

$$
s=t \in \mathbb{Z}, t<p, t \equiv \epsilon \quad(\bmod 2) .
$$

Now, we turn our attention to the normalization factor $r_{1}(t)$. We see that $\Gamma\left(\left(1+s-p+\epsilon^{\prime}\right) / 2\right)=\infty$ if and only if $\left(1+s-p+\epsilon^{\prime}\right) / 2 \in \mathbb{Z}_{\leq 0}$. This is equivalent with $s=t \in \mathbb{Z}, 1+t-p+\epsilon^{\prime} \leq 0, t \equiv \epsilon(\bmod 2)$. It is easy to see 
that this is equivalent with (7.8). Now, the $B_{1}(t)$ has a (simple) pole if and only if $\Gamma\left(\left(s-p+\epsilon^{\prime}\right) / 2\right)=\infty$. This proves (i).

\section{IRREDUCIBLE $(\mathfrak{s} p(2, \mathbb{R}), U(2))$-MODULES}

In this section we list irreducible $(\mathfrak{s} p(2, \mathbb{R}), U(2))$-modules using the Langlands classification $([10])$. We describe the set of equivalence classes as follows:

(min) Langlands quotients supported on the minimal parabolic subgroup:

$$
\operatorname{Lang}\left(||^{s_{1}} \operatorname{sgn}^{\epsilon_{1}} \times||^{s_{2}} \operatorname{sgn}^{\epsilon_{2}} \rtimes \mathbf{1}\right) \text {, }
$$

where $\left(s_{1}, s_{2}, \epsilon_{1}, \epsilon_{2}\right) \in \mathbb{C} \times \mathbb{C} \times\{0,1\} \times\{0,1\}, \operatorname{Re}\left(s_{1}\right) \geq \operatorname{Re}\left(s_{2}\right) \geq 0$ and $\operatorname{Re}\left(s_{1}\right)+\operatorname{Re}\left(s_{2}\right)>0$; the infinitesimal is $\left(s_{1}, s_{2}\right)$.

(sig) Langlands quotients supported on the Siegel maximal parabolic subgroup:

$$
\operatorname{Lang}\left(\delta\left(||^{s}, k\right) \rtimes \mathbf{1}\right) \simeq \operatorname{Lang}\left(\delta\left(||^{s} \operatorname{sgn}, k\right) \rtimes \mathbf{1}\right),
$$

$(s, k) \in \mathbb{C} \times \mathbb{Z}_{>0}, \operatorname{Re}(s)>0$; the infinitesimal is $(s+k / 2, s-k / 2)$

(non-sig) Langlands quotients supported on the non-Siegel maximal parabolic subgroup:

$$
\operatorname{Lang}\left(||^{s} \operatorname{sgn}^{\epsilon} \rtimes X(p, w)\right) \text {, }
$$

$(s, \epsilon, p, w) \in \mathbb{C} \times\{0,1\} \times \mathbb{Z}_{\geq 0} \times\{ \pm\}, \operatorname{Re}(s)>0$; the infinitesimal is $(s, p)$.

(dis) Discrete series for $S p(4, \mathbb{R})$ have regular and integral infinitesimal characters. This means that after a conjugation by an element of the Weyl group the infinitesimal character is of the form $(p, t) \in \mathbb{Z} \times \mathbb{Z}, p>t>0$. There are four non-equivalent discrete series having the infinitesimal character $(p, t) \in \mathbb{Z} \times \mathbb{Z}, p>t>0$. We describe them using [10, Theorem 9.20]. We use the standard realization of roots found in [3]. In particular, $(1,-1)$ is a simple compact root and $(0,2)$ is other root for $C_{2}$-system. According to [10, Theorem 9.20], those discrete series are determined by their restriction to $U(2)$ :

1. $U(2)$-type $(p, t)+(2,1)-(1,-1)=(p+1, t+2)$ is contained with multiplicity one. All other $U(2)$-types are of the form $(p+1, t+2)+$ $n(1,-1)+m(0,2)=(p+n+1, t+2-n+2 m)$, for some $m, n \in \mathbb{Z}_{\geq 0}$. We denote this representation by $X(p, t)$.

2. $U(2)$-type $(p,-t)+(2,-1)-(1,-1)=(p+1,-t)$ is contained with multiplicity one. All other $U(2)$-types are of the form $(p+1,-t)+$ $n(1,1)+m(0,-2)=(p+n+1,-t+n-2 m)$, for some $m, n \in \mathbb{Z}_{\geq 0}$. We denote this representation by $X(p,-t)$.

3. $U(2)$-type $(t,-p)+(1,-2)-(1,-1)=(t,-p-1)$ is contained with multiplicity one. All other $U(2)$-types are of the form $(t,-p-1)+$ $n(-1,-1)+m(2,0)=(t-n+2 m,-p-1-n)$, for some $m, n \in \mathbb{Z}_{\geq 0}$. We denote this representation by $X(t,-p)$.

4. $U(2)$-type $(-t,-p)+(-1,-2)-(1,-1)=(-t-2,-p-1)$ is contained with multiplicity one. All other $U(2)$-types are of the form $(-t-2,-p-$ 
1) $+n(1,-1)+m(-2,0)=(-t-2+n-2 m,-p-1-n)$, for some $m, n \in \mathbb{Z}_{\geq 0}$. We denote this representation by $X(-t,-p)$.

(limit-dis) Limits of discrete series for $S p(4, \mathbb{R})([10$, Theorem 12.26]) have infinitesimal characters, that after a conjugation by an element of the Weyl group, are of the form $(p, t) \in \mathbb{Z} \times \mathbb{Z}$, where either $p=t>0$ or $p>t=0$. Now, ([10], Theorem 12.26) shows that we may define them as follows. Let $\left(m_{1}, m_{2}\right) \in \mathbb{Z}^{2}, m_{1}>m_{2}>0$. When $p=t>0$, we have $\psi_{(p, p)}^{\left(p+m_{1}, p+m_{2}\right)}(X(p+$ $\left.\left.m_{1}, p+m_{2}\right)\right)=0$ and $\psi_{(p, p)}^{\left(p+m_{1}, p+m_{2}\right)}\left(X\left(-p-m_{2},-p-m_{1}\right)\right)=0$ since the parameter $(p, p)$ is orthogonal to the compact root $(1,-1)$. We write

$$
\begin{aligned}
& X^{1}(p,-p)=\psi_{(p, p)}^{\left(p+m_{1}, p+m_{2}\right)}\left(X\left(p+m_{1},-p-m_{2}\right)\right) \neq 0, \\
& X^{2}(p,-p)=\psi_{(p, p)}^{\left(p+m_{1}, p+m_{2}\right)}\left(X\left(p+m_{2},-p-m_{1}\right)\right) \neq 0,
\end{aligned}
$$

for the limits of discrete series for the cases (2) and (3), respectively. Their $U(2)$-type structure is as in (2) and (3), respectively, but for $p=t$.

Similarly, for $p>t=0$, we get four limits of discrete series for the cases described by (1)-(4):

$$
\begin{aligned}
& X^{1}(p, 0)=\psi_{(p, 0)}^{\left(p+m_{1}, m_{2}\right)}\left(X\left(p+m_{1}, m_{2}\right)\right), \\
& X^{2}(p, 0)=\psi_{(p, 0)}^{\left(p+m_{1}, m_{2}\right)}\left(X\left(p+m_{1},-m_{2}\right)\right), \\
& X^{1}(0,-p)=\psi_{(p, 0)}^{\left(p+m_{1}, m_{2}\right)}\left(X\left(m_{2},-p-m_{1}\right)\right), \\
& X^{2}(0,-p)=\psi_{(p, 0)}^{\left(p+m_{1}, m_{2}\right)}\left(X\left(-m_{2},-p-m_{1}\right)\right) .
\end{aligned}
$$

(temp) Tempered representations that are not discrete series or limits of discrete series are fully induced from discrete series or limits of discrete series of Levi factors by the work of Knapp-Zuckerman ([10]). Thus, except irreducible unitary principal series (see Lemma 5.1) we have the following:

LEMMA 8.1. Let $\eta$ be a unitary character of $\mathbb{R}^{\times}, p \in \mathbb{Z}_{\geq 0}$, and $k \in \mathbb{Z}_{>0}$. Then we have the following:

(i) $\eta \rtimes X(p, w), w \in\{ \pm\}$, is reducible if and only if $\eta=1$ and $p>0$ :

$$
\begin{aligned}
& 1 \rtimes X(p,+) \simeq X^{1}(p, 0) \oplus X^{2}(p, 0), \\
& 1 \rtimes X(p,-) \simeq X^{1}(0,-p) \oplus X^{2}(0,-p) .
\end{aligned}
$$

(ii) $\delta(\eta, k) \rtimes \mathbf{1}$ is reducible if and only if $\eta \in\{1$, sgn $\}$ and $k$ is even. Moreover, for $p>0, \delta(1,2 p) \rtimes \mathbf{1}=\delta(\operatorname{sgn}, 2 p) \rtimes \mathbf{1} \simeq X^{1}(p,-p) \oplus X^{2}(p,-p)$.

Proof. This follows from the classification of tempered representations done by Knapp-Zuckerman [10], Langlands disjointness theorem ([10], Theorem 14.90), and Corollary 5.2. In fact, reducibilities are Schmid's identities. 


\section{Composition SERIES IN THE CASE OF INTEGRAL INFINITESIMAL CHARACTER I}

In this section we begin the description of the composition series of generalized and degenerate principal series having integral infinitesimal characters $(p, t) \in \mathbb{Z} \times \mathbb{Z}$. Up to a conjugation by an element of the Weyl group we see that there are four types of the principal series $\left|{ }^{p} \operatorname{sgn}^{\epsilon_{1}} \times\right| \mid{ }^{t} \operatorname{sgn}^{\epsilon_{2}} \rtimes \mathbf{1}$ :

$$
\left.\left(\epsilon_{1}, \epsilon_{2}\right) \equiv(p, t),(p, t+1),(p+1, t),(p+1, t+1) \quad(\bmod 2) \mathbb{Z} \times 2 \mathbb{Z}\right)
$$

with that infinitesimal character. We also assume that

$$
p \geq t \geq 0 \text { and } p+t>0 .
$$

The first condition in (9.2) is obtained applying a conjugation by a suitable element of the Weyl group. Then the second condition means that we do not consider the case $p=t=0$ here since it is treated in Lemma 5.1 and Corollary 5.2.

LEMMA 9.1. | $\left.\right|^{p} \operatorname{sgn}^{p} \times||^{t} \operatorname{sgn}^{t} \rtimes \mathbf{1}$ is irreducible.

Proof. This follows from Lemma 5.1.

Also, Theorems 2.4 and 2.5 imply that in $\mathcal{R}(S p(4, \mathbb{R}))$ we have the following (we let $V_{0}=0$ ):

$$
\begin{aligned}
& ||^{p} \operatorname{sgn}^{p+1} \times||^{t} \operatorname{sgn}^{t+1} \rtimes \mathbf{1} \\
& \quad=||^{t} \operatorname{sgn}^{t+1} \rtimes||^{p} \operatorname{sgn}^{p+1} \rtimes \mathbf{1} \\
& \quad=||^{p} \operatorname{sgn}^{p+1} \rtimes(X(t,-)+X(t,+))+||^{p} \operatorname{sgn}^{p+1} \rtimes V_{t} \\
& \quad=||^{t} \operatorname{sgn}^{t+1} \rtimes(X(p,-)+X(p,+))+||^{t} \operatorname{sgn}^{t+1} \rtimes V_{p},
\end{aligned}
$$

||$^{p} \operatorname{sgn}^{p+1} \times||^{t} \operatorname{sgn}^{t} \rtimes \mathbf{1}=||^{p} \operatorname{sgn}^{p+1} \times||^{-t} \operatorname{sgn}^{t} \rtimes \mathbf{1}$

$$
\begin{aligned}
& =\delta\left(||^{(p-t) / 2} \operatorname{sgn}^{t}, p+t\right) \rtimes \mathbf{1}+\zeta\left(||^{(p-t) / 2} \operatorname{sgn}^{t}, p+t\right) \rtimes \mathbf{1} \\
& =\delta\left(||^{(p+t) / 2} \operatorname{sgn}^{t}, p-t\right) \rtimes \mathbf{1}+\zeta\left(||^{(p+t) / 2} \operatorname{sgn}^{t}, p-t\right) \rtimes \mathbf{1} \quad(p>t) \\
& =||^{t} \operatorname{sgn}^{t} \rtimes X(p,+)+||^{t} \operatorname{sgn}^{t} \rtimes X(p,-)+||^{t} \operatorname{sgn}^{t} \rtimes V_{p},
\end{aligned}
$$

and

$(9.5)$

$$
\begin{aligned}
& ||^{t} \operatorname{sgn}^{t+1} \times||^{p} \operatorname{sgn}^{p} \rtimes \mathbf{1}=||^{p} \operatorname{sgn}^{p} \rtimes||^{-t} \operatorname{sgn}^{t+1} \rtimes \mathbf{1} \\
& \quad=\delta\left(||^{(p-t) / 2} \operatorname{sgn}^{t+1}, p+t\right) \rtimes \mathbf{1}+\zeta\left(||^{(p-t) / 2} \operatorname{sgn}^{t+1}, p+t\right) \rtimes \mathbf{1} \\
& \quad=\delta\left(||^{(p+t) / 2} \operatorname{sgn}^{t+1}, p-t\right) \rtimes \mathbf{1}+\zeta\left(||^{(p+t) / 2} \operatorname{sgn}^{t+1}, p-t\right) \rtimes \mathbf{1} \quad(p>t) \\
& \quad=||^{p} \operatorname{sgn}^{p} \rtimes X(t,+)+||^{p} \operatorname{sgn}^{p} \rtimes X(t,-)+||^{p} \operatorname{sgn}^{p} \rtimes V_{t} .
\end{aligned}
$$


We note that Theorem 2.5 (ii) implies that

$$
\begin{aligned}
& \delta\left(||^{(p-t) / 2} \operatorname{sgn}^{t}, p+t\right) \simeq \delta\left(||^{(p-t) / 2} \operatorname{sgn}^{t+1}, p+t\right), \\
& \delta\left(||^{(p+t) / 2} \operatorname{sgn}^{t}, p-t\right) \simeq \delta\left(||^{(p+t) / 2} \operatorname{sgn}^{t+1}, p-t\right) .
\end{aligned}
$$

Hence, in $\mathcal{R}(S p(4, \mathbb{R})$ ) (the last step follows using the discussion before the proof of Lemma 7.2), we have the following:

$$
\begin{aligned}
\delta\left(||^{(p-t) / 2} \operatorname{sgn}^{t}, p+t\right) \rtimes \mathbf{1} & =\delta\left(||^{(p-t) / 2} \operatorname{sgn}^{p}, p+t\right) \rtimes \mathbf{1} \\
& =\delta\left(||^{(t-p) / 2} \operatorname{sgn}^{p}, p+t\right) \rtimes \mathbf{1} .
\end{aligned}
$$

The decompositions (9.3), (9.4), and (9.5) will help us to determine principal series where non-tempered irreducible subquotients having infinitesimal character $(p, t)$ appear as subquotients. We have the following lemma:

LEMma 9.2. Assume that (9.2) holds. Then, we have the following:

(i) ||$^{p} \operatorname{sgn}^{p} \times||^{t} \operatorname{sgn}^{t} \rtimes \mathbf{1} \simeq \operatorname{Lang}\left(\left.\left|{ }^{p} \operatorname{sgn}^{p} \times\right|\right|^{t} \operatorname{sgn}^{t} \rtimes \mathbf{1}\right)$.

(ii) Up to multiplicity, all non-tempered irreducible subquotients of the representation $\left.\left|{ }^{p} \operatorname{sgn}^{p+1} \times\right|\right|^{t} \operatorname{sgn}^{t+1} \rtimes \mathbf{1}$ are the following:

$$
\begin{aligned}
& \operatorname{Lang}\left(||^{t} \operatorname{sgn}^{t+1} \rtimes X(p,+)\right), \operatorname{Lang}\left(||^{t} \operatorname{sgn}^{t+1} \rtimes X(p,-)\right), \\
& \operatorname{Lang}\left(||^{p} \operatorname{sgn}^{p+1} \rtimes X(t,+)\right), \operatorname{Lang}\left(||^{p} \operatorname{sgn}^{p+1} \rtimes X(t,-)\right), \\
& \left.\operatorname{Lang}\left(||^{p} \operatorname{sgn}^{p+1} \rtimes||^{t} \operatorname{sgn}^{t+1} \rtimes \mathbf{1}\right) \text { (multiplicity one }\right) .
\end{aligned}
$$

(iii) Up to multiplicity, all non-tempered irreducible subquotients of the representation ||$^{p} \operatorname{sgn}^{p+1} \times||^{t} \operatorname{sgn}^{t} \rtimes \mathbf{1}$ and $\left|{ }^{t} \operatorname{sgn}^{t+1} \times\right|{ }^{p} \operatorname{sgn}^{p} \rtimes \mathbf{1}$ are the following:

$$
\begin{aligned}
& \operatorname{Lang}\left(||^{p} \operatorname{sgn}^{p} \rtimes X(t,+)\right), \operatorname{Lang}\left(||^{p} \operatorname{sgn}^{p} \rtimes X(t,-)\right), \\
& \operatorname{Lang}\left(||^{t} \operatorname{sgn}^{t} \rtimes X(p,+)\right), \operatorname{Lang}\left(||^{t} \operatorname{sgn}^{t} \rtimes X(p,-)\right), \\
& \operatorname{Lang}\left(\delta\left(||^{(p+t) / 2} \operatorname{sgn}^{t}, p-t\right) \rtimes \mathbf{1}\right), \operatorname{Lang}\left(\delta\left(||^{(p-t) / 2} \operatorname{sgn}^{t}, p+t\right) \rtimes \mathbf{1}\right), \\
& \operatorname{Lang}\left(||^{p} \operatorname{sgn}^{p+1} \times \mid{ }^{t} \operatorname{sgn}^{t} \rtimes \mathbf{1}\right), \\
& \quad\left(\text { only in }||^{p} \operatorname{sgn}^{p+1} \times||^{t} \operatorname{sgn}^{t} \rtimes \mathbf{1} ; \text { multiplicity one }\right), \\
& \operatorname{Lang}\left(||^{p} \operatorname{sgn}^{p} \times||^{t} \operatorname{sgn}^{t+1} \rtimes \mathbf{1}\right), \\
& \quad\left(\text { only in }||^{t} \operatorname{sgn}^{t+1} \times||^{p} \operatorname{sgn}^{p} \rtimes \mathbf{1} ; \text { multiplicity one }\right) .
\end{aligned}
$$

Proof. Using the list of irreducible representations given in Section 8, it is easy to write down all irreducible non-tempered representations having infinitesimal character $(p, t)$. They are given by (i), (ii) and (iii) the above. Also, Lemma 9.1, (9.3), (9.4), and (9.5) describe some principal series where they appear as subquotients. Next, (i) follows from Lemma 9.1, and irreducible representation from (i) cannot appear as an irreducible subquotient of principal series considered in (ii) and (iii) because of Theorem2.3. Finally, to complete the proof of (ii) and (iii), we use $U(2)$-types. More precisely, 
Lemma 6.1 implies that the principal series $\left.\left|{ }^{p} \operatorname{sgn}^{p+1} \times\right|\right|^{t} \operatorname{sgn}^{t+1} \rtimes \mathbf{1}$ does not have any $U(2)$-type common with those of ||$^{p} \operatorname{sgn}^{p+1} \times||^{t} \operatorname{sgn}^{t} \rtimes \mathbf{1}$ and ||$^{t} \operatorname{sgn}^{t+1} \times||^{p} \operatorname{sgn}^{p} \rtimes \mathbf{1}$.

Now, we list all tempered irreducible representations of infinitesimal character $(p, t)$ and determine principal series where they appear as subquotients. We have the following lemma:

Lemma 9.3. Assume that (9.2) holds. Then we have the following:

(i) There are no tempered irreducible subquotients in ||$^{p} \operatorname{sgn}^{p} \times||^{t} \operatorname{sgn}^{t} \rtimes \mathbf{1}$, while for ||$^{p} \operatorname{sgn}^{p+1} \times||^{t} \operatorname{sgn}^{t+1} \rtimes \mathbf{1}$ only possible tempered irreducible subquotients appear when $p>t=0$ and they are $\operatorname{sgn} \rtimes X(p, w)(w \in$ $\{ \pm\}$ ) (they are irreducible by Lemma 8.1).

(ii) Up to multiplicity, all tempered irreducible subquotients of $\mid{ }^{p} \operatorname{sgn}^{p+1} \times$ $\mid{ }^{t} \operatorname{sgn}^{t} \rtimes \mathbf{1}$ and ||$^{t} \operatorname{sgn}^{t+1} \times||^{p} \operatorname{sgn}^{p} \rtimes \mathbf{1}$ are the following:

1. $(p>t>0) X(p, t), X(p,-t), X(t,-p)$, and $X(-t,-p)$;

2. $(p>t=0) X^{1}(p, 0), X^{2}(p, 0), X^{1}(0,-p)$, and $X^{2}(0,-p)$;

3. $(p=t>0) X^{1}(p,-p)$ and $X^{2}(p,-p)$.

Moreover, any of them must appear in ||$^{p} \operatorname{sgn}^{p+1} \times||^{t} \operatorname{sgn}^{t} \rtimes \mathbf{1}$ or in $\left.\left|{ }^{t} \operatorname{sgn}^{t+1} \times\right|\right|^{p} \operatorname{sgn}^{p} \rtimes \mathbf{1}$.

PROOF. Using the list of irreducible representations from Section 8 it is easy to see that all tempered irreducible representations having infinitesimal character $(p, t)$ are those listed in (i) and (ii) of the statement of Lemma 9.3. Next, according to Theorem 2.1 (iii) any irreducible tempered representation having infinitesimal character $(p, t)$ must be a subquotient of one of our four principal series mentioned in Lemma 9.2. Since ||$^{p} \operatorname{sgn}^{p} \times||{ }^{t} \operatorname{sgn}^{t} \rtimes \mathbf{1}$ is irreducible (see Lemma 9.1) it cannot contain tempered representations. Next, those listed in (ii), using the description of $U(2)$-types of them (see (dis) and (limit-dis) in Section 8) and Lemma 6.1, cannot appear in ||$^{p} \operatorname{sgn}^{p+1} \times$ $\mid{ }^{t} \operatorname{sgn}^{t+1} \rtimes \mathbf{1}$. Those, listed in (i) obviously appear $\mid{ }^{p} \operatorname{sgn} n^{p+1} \times \operatorname{sgn} \rtimes \mathbf{1}$, while they cannot appear in ||$^{p} \operatorname{sgn}^{p+1} \times \mid{ }^{t} \operatorname{sgn}^{t} \rtimes \mathbf{1}$ and ||${ }^{t} \operatorname{sgn}^{t+1} \times||^{p} \operatorname{sgn}^{p} \rtimes \mathbf{1}$ $(t=0)$ for the same reason.

Now, we begin to study the composition series of generalized and degenerate principal series having infinitesimal character $(p, t)$. Lemma 9.1 completes the investigation of the first case in (9.1). In this section we consider the last case in (9.1). The remaining two cases are more complicated and they will be treated in Sections 10 and 11.

Lemma 9.4. Assume that $p>t>0$. Let $w \in\{ \pm\}$. Then we have the following:

||$^{t} \operatorname{sgn}^{t+1} \rtimes X(p, w) \hookrightarrow||^{p} \operatorname{sgn}^{p+1} \rtimes X(t, w) \rightarrow \operatorname{Lang}\left(||^{p} \operatorname{sgn}^{p+1} \rtimes X(t, w)\right)$. 
Also, in $\mathcal{R}(S p(4, \mathbb{R}))$, we have the following (the composition series is obvious):

$$
\begin{aligned}
||^{t} \operatorname{sgn}^{t+1} \rtimes X(p, w)= & \operatorname{Lang}\left(||^{t} \operatorname{sgn}^{t+1} \rtimes X(p, w)\right), \\
\left.||\right|^{p} \operatorname{sgn}^{p+1} \rtimes X(t, w)= & \left.\operatorname{Lang}\left(||^{t} \operatorname{sgn}^{t+1} \rtimes X(p, w)\right)\right) \\
& +\operatorname{Lang}\left(||^{p} \operatorname{sgn}^{p+1} \rtimes X(t, w)\right), \\
||^{p} \operatorname{sgn}^{p+1} \rtimes V_{t}= & \operatorname{Lang}\left(||^{p} \operatorname{sgn}^{p+1} \rtimes||^{t} \operatorname{sgn}^{t+1} \rtimes \mathbf{1}\right) .
\end{aligned}
$$

Finally, $\mid{ }^{t}$ sgn $^{t+1} \rtimes V_{p}$ has the following composition series $V_{0} \subset V_{1}=$ | ${ }^{t} \operatorname{sgn}^{t+1} \rtimes V_{p}$, where

$$
\begin{aligned}
& V_{0} \simeq \operatorname{Lang}\left(||^{p} \operatorname{sgn}^{p+1} \rtimes X(t,+)\right) \oplus \operatorname{Lang}\left(||^{p} \operatorname{sgn}^{p+1} \rtimes X(t,-)\right), \\
& V_{1} / V_{0} \simeq \operatorname{Lang}\left(||^{p} \operatorname{sgn}^{p+1} \times||^{t} \operatorname{sgn} n^{t+1} \rtimes \mathbf{1}\right) .
\end{aligned}
$$

Proof. We use Lemma 7.3 (in our case we have $\eta_{u}=s g n^{t+1}$ ). Now, in the commutative diagram (7.7), $B_{1}(t)$ has a simple pole. We write $B_{1}$ for the corresponding residue operator. Put $B_{i}=B_{i}(t), i=2,3$. Now, since $B(t)$ is holomorphic, we obtain the following diagram:

$$
\begin{aligned}
\oplus_{\varepsilon \in\{ \pm\}}||^{t} \operatorname{sgn}^{t+1} \rtimes X(p, \varepsilon) \stackrel{i}{\longrightarrow} & ||^{t} \operatorname{sgn}^{t+1} \times||^{p} \operatorname{sgn}^{p+1} \rtimes \mathbf{1} \\
B_{1} \mid & ||^{p} \operatorname{sgn}^{p+1} \times||^{t} \operatorname{sgn}^{t+1} \rtimes \mathbf{1} \\
B_{2} \downarrow & ||^{p} \operatorname{sgn}^{p+1} \times||^{-t} \operatorname{sgn}^{t+1} \rtimes \mathbf{1} \\
B_{3} \downarrow & \\
\oplus_{\varepsilon \in\{ \pm\}}||^{-t} \operatorname{sgn}^{t+1} \rtimes X(p, \varepsilon) \stackrel{j}{\longrightarrow} & ||^{-t} \operatorname{sgn}^{t+1} \times||^{p} \operatorname{sgn}^{p+1} \rtimes \mathbf{1},
\end{aligned}
$$

Now, Theorems 2.4 and 2.5 show that $B_{1}$ and $B_{3}$ are isomorphisms. Also, hence the commutative diagram (9.9) shows that

$$
||^{t} \operatorname{sgn}^{t+1} \rtimes X(p,+) \oplus||^{t} \operatorname{sgn}^{t+1} \rtimes X(p,-) \hookrightarrow \operatorname{ker} B_{2} .
$$

Applying Theorem 2.4, it is easy to compute ker $B_{2}$. We obtain the following:

$$
\begin{aligned}
& ||^{t} \operatorname{sgn}^{t+1} \rtimes X(p,+) \oplus||^{t} \operatorname{sgn}^{t+1} \rtimes X(p,-) \\
& \quad \hookrightarrow||^{p} \operatorname{sgn}^{p+1} \rtimes X(t,+) \oplus||^{p} \operatorname{sgn}^{p+1} \rtimes X(t,-)=\operatorname{ker} B_{2} .
\end{aligned}
$$


Next, we the following diagram:

$$
\begin{aligned}
\left|{ }^{p} \operatorname{sgn}^{p+1} \times\right|{ }^{t} \operatorname{sgn}^{t+1} \rtimes \mathbf{1} & \stackrel{B_{1}^{-1}}{\longrightarrow}||^{t} \operatorname{sgn}^{t+1} \times \mid{ }^{p} \operatorname{sgn}^{p+1} \rtimes \mathbf{1} \\
& \stackrel{J_{1}}{\longrightarrow}||^{t} \operatorname{sgn}^{t+1} \times||^{-p} \operatorname{sgn}^{p+1} \rtimes \mathbf{1} \\
& \simeq||^{-p} \operatorname{sgn}^{p+1} \times \mid{ }^{t} \operatorname{sgn}^{t+1} \rtimes \mathbf{1} \\
& \stackrel{J_{2}}{\longrightarrow}||^{-p} \operatorname{sgn}^{p+1} \times||^{-t} \operatorname{sgn}^{t+1} \rtimes \mathbf{1},
\end{aligned}
$$

where we choose some non-zero intertwining operators $J_{1}$ and $J_{2}$ (induced long-intertwining operators). The image of the composition of all intertwining operators is isomorphic to $\operatorname{Lang}\left(||{ }^{p} \operatorname{sgn}^{p+1} \times||{ }^{t} \operatorname{sgn}^{t+1} \rtimes \mathbf{1}\right)$ as it follows from the Langlands classification.

Next, by using embedding in (9.10), we obtain

$$
\begin{aligned}
\operatorname{ker} J_{1} & =||^{t} \operatorname{sgn}^{t+1} \rtimes X(p,+) \oplus||^{t} \operatorname{sgn}^{t+1} \rtimes X(p,-) \\
& \subset B_{1}^{-1}\left(||^{p} \operatorname{sgn}^{p+1} \rtimes X(t,+) \oplus||^{p} \operatorname{sgn}^{p+1} \rtimes X(t,-)\right) .
\end{aligned}
$$

Next, (9.11) gives an analogue of (7.7) for ||$^{p} \operatorname{sgn}^{p+1} \rtimes X(t,+) \oplus$ ||$^{p} \operatorname{sgn}^{p+1} \rtimes X(t,-)$ (observe no pole situation since $p>t$ by our assumption). Hence, the composition of the first three intertwining operators in (9.11) has the image isomorphic to $\operatorname{Lang}\left(||^{p} \operatorname{sgn}^{p+1} \rtimes X(t,+)\right) \oplus \operatorname{Lang}\left(||^{p} \operatorname{sgn}^{p+1} \rtimes\right.$ $X(t,-))$. Thus, (9.12) implies

$$
\begin{aligned}
& B_{1}^{-1}\left(||^{p} \operatorname{sgn}^{p+1} \rtimes X(t,+) \oplus||^{p} \operatorname{sgn}^{p+1} \rtimes X(t,-)\right) / \operatorname{ker} J_{1} \\
& \quad \simeq \operatorname{Lang}\left(||^{p} \operatorname{sgn}^{p+1} \rtimes X(t,+)\right) \oplus \operatorname{Lang}\left(||^{p} \operatorname{sgn}^{p+1} \rtimes X(t,-)\right) .
\end{aligned}
$$

This implies

$$
\begin{aligned}
\oplus_{w \in\{ \pm\}}||^{t} \operatorname{sgn}^{t+1} \rtimes X(p, w) & \hookrightarrow \oplus_{w \in\{ \pm\}}||^{p} \operatorname{sgn}^{p+1} \rtimes X(t, w) \\
& \rightarrow \oplus_{w \in\{ \pm\}} \operatorname{Lang}\left(||^{p} \operatorname{sgn}^{p+1} \rtimes X(t, w)\right) .
\end{aligned}
$$

Now, (9.14) implies the following. If $\operatorname{Lang}\left(||^{t} \operatorname{sgn}^{t+1} \rtimes X(p, w)\right)$ is a subquotient of ||$^{p} \operatorname{sgn}^{p+1} \rtimes X\left(t, w^{\prime}\right)$, then

$$
||^{t} \operatorname{sgn}^{t+1} \rtimes X(p, w) \hookrightarrow||^{p} \operatorname{sgn}^{p+1} \rtimes X\left(t, w^{\prime}\right) .
$$

Now, we restrict $(9.15)$ to $U(2)$ and apply Lemma 6.1 to obtain $w=w^{\prime}$. This proves (9.7). Now, Lemma 9.2 (ii), Lemma 9.3, and (9.7) imply the first two equalities in (9.8). The composition series of ||$^{t} \operatorname{sgn}^{t+1} \rtimes V_{p}$ follows from (9.11). In more detail, (9.13) and (9.7) imply

$$
\begin{gathered}
\operatorname{Lang}\left(||{ }^{p} \operatorname{sgn}^{p+1} \rtimes X(t,+)\right) \oplus \operatorname{Lang}\left(||^{p} \operatorname{sgn}^{p+1} \rtimes X(t,-)\right) \\
\hookrightarrow \operatorname{Im} J_{1} \simeq||{ }^{t} \operatorname{sgn}^{t+1} \rtimes V_{p} .
\end{gathered}
$$

Next, we send $\operatorname{Im} J_{1}$ by the isomorphism in (9.11), and then we intersect the image with ker $J_{2}$, which is isomorphic to

$$
||^{-p} \operatorname{sgn}^{p+1} \rtimes X(t,-) \oplus||^{-p} \operatorname{sgn}^{p+1} \rtimes X(t,+) .
$$


If this intersection is not exactly equal to the direct sum in (9.16), then the first and second formula in (9.8) (proved above) show that ||$^{t} s g n^{t+1} \rtimes V_{p}$ contains ||$^{t} \operatorname{sgn}^{t+1} \rtimes X(p, w)$, for some $w \in\{ \pm\}$. This is not possible since $\mid{ }^{t} \operatorname{sgn}^{t+1} \rtimes X(p, w)$ is large, while ||$^{t} \operatorname{sgn}^{t+1} \rtimes V_{p}$ cannot contain a large subquotient (we may also use Lemma 6.1 to obtain the same conclusion). Now, since the image of the composition of all intertwining operators in (9.7) is isomorphic to $\operatorname{Lang}\left(||^{p} \operatorname{sgn}^{p+1} \times||^{t} \operatorname{sgn}^{t+1} \rtimes \mathbf{1}\right)$, as it follows from the Langlands classification, we obtain the composition series. Finally, the fourth formula follows from (9.3) combined with already proved the first two equalities in (9.8), together with the composition series of ||$^{t} \operatorname{sgn}^{t+1} \rtimes V_{p}$.

The other two lemmas have similar, but easier proofs. We leave to the reader to make necessary modifications.

Lemma 9.5. Assume that $p=t>0$. In $\mathcal{R}(S p(4, \mathbb{R}))$, we have the following $(w \in\{ \pm\})$ :

$$
\begin{aligned}
& \mid{ }^{p} \operatorname{sgn}^{p+1} \rtimes X(p, w)=\operatorname{Lang}\left(||^{p} \operatorname{sgn}^{p+1} \rtimes X(p, w)\right), \\
& ||{ }^{p} \operatorname{sgn}^{p+1} \rtimes V_{p}=\operatorname{Lang}\left(||^{p} \operatorname{sgn}^{p+1} \times||^{p} \operatorname{sgn}^{p+1} \rtimes \mathbf{1}\right) .
\end{aligned}
$$

Lemma 9.6. Assume that $p>t=0$. Then, in $\mathcal{R}(S p(4, \mathbb{R}))$, we have the following $(w \in\{ \pm\})$ :

$\operatorname{sgn} \rtimes X(p, w)$ is irreducible

||$^{p} \operatorname{sgn}^{p+1} \rtimes X(0, w)=\operatorname{sgn} \rtimes X(p, w)+\operatorname{Lang}\left(||^{p} \operatorname{sgn}^{p+1} \rtimes X(0, w)\right)$.

Also, we have the following:

$$
\operatorname{sgn} \rtimes V_{p} \simeq \operatorname{Lang}\left(||^{p} \operatorname{sgn}^{p+1} \rtimes X(0,+)\right) \oplus \operatorname{Lang}\left(||^{p} \operatorname{sgn}^{p+1} \rtimes X(0,-)\right) \text {. }
$$

10. Composition SERIES IN THE CASE OF INTEGRAL INFINITESIMAL CHARACTER II

In this section we determine the structure of generalized and degenerate principal series of ||$^{p} \operatorname{sgn}^{p+1} \times||^{t} \operatorname{sgn}^{t} \rtimes \mathbf{1}$. We assume that (9.2) holds. We start with the next two theorems where we describe the composition series of all generalized principal series appearing in (9.4).

TheOREM 10.1. Assume $p>t>0$. Then the following sequences are exact:

$$
\begin{aligned}
X(p,-t) \oplus X(t,-p) & \hookrightarrow \delta\left(||^{(p-t) / 2} \operatorname{sgn}^{t}, p+t\right) \rtimes \mathbf{1} \\
& \rightarrow \operatorname{Lang}\left(\delta\left(||^{(p-t) / 2} \operatorname{sgn}^{t}, p+t\right) \rtimes \mathbf{1}\right) .
\end{aligned}
$$

$(10.2)$

$$
\begin{aligned}
& X(p, t) \oplus X(p,-t) \hookrightarrow||^{t} \operatorname{sgn}^{t} \rtimes X(p,+) \rightarrow \operatorname{Lang}\left(||^{t} \operatorname{sgn}^{t} \rtimes X(p,+)\right) \\
& X(t,-p) \oplus X(-t,-p) \hookrightarrow||^{t} \operatorname{sgn}^{t} \rtimes X(p,-) \rightarrow \operatorname{Lang}\left(||^{t} \operatorname{sgn}^{t} \rtimes X(p,-)\right) .
\end{aligned}
$$

Moreover, $X(p,-t)$ and $X(t,-p)$ are large ([29]). 
Proof. We begin by showing that $\operatorname{Lang}\left(\delta\left(||^{(p-t) / 2} \operatorname{sgn}^{t}, p+t\right) \rtimes \mathbf{1}\right)$ is only non-tempered irreducible subquotient of $\delta\left(||^{(p-t) / 2} \operatorname{sgn}^{t}, p+t\right) \rtimes \mathbf{1}$. If not, then the inequality in Theorem 2.3 and Lemma 9.2 (iii) show that some other non-tempered irreducible subquotient must be of the form $\operatorname{Lang}\left(||^{t} \operatorname{sgn}^{t} \rtimes X(p, w)\right), w \in\{ \pm\}$. Now, Lemma 9.2 (iii) implies that $\operatorname{Lang}\left(||^{t} \operatorname{sgn}^{t} \rtimes X(p, w)\right)$ is only non-tempered irreducible subquotient of ||$^{t} \operatorname{sgn}^{t} \rtimes X(p, w)$ since only other possible is $\delta\left(||^{(p-t) / 2} \operatorname{sgn}^{t}, p+t\right) \rtimes \mathbf{1}$. Next, Lemma 9.3 (ii) implies that other possible irreducible subquotients of ||$^{t} \operatorname{sgn}^{t} \rtimes X(p, w)$ are discrete series $X(p, t), X(p,-t), X(t,-p)$, and $X(-t,-p)$. Now, using Lemma 6.1, we see that $(p+1, t)$ (resp., $(-t,-p-1))$ appears in ||$^{t} \operatorname{sgn}^{t} \rtimes X(p,+)$ (resp., ||$\left.^{t} \operatorname{sgn}^{t} \rtimes X(p,-)\right)$ with multiplicity one. Next, the description of $U(2)$-types of discrete series (see Section 8, (dis)) shows that those types do not appear in discrete series. Hence, if $w=+$ (resp., $w=-)$, then we see that the type $(p+1, t)$ (resp., $(-t,-p-1))$ appears in $\operatorname{Lang}\left(||^{t} \operatorname{sgn}^{t} \rtimes X(p,+)\right)$ (resp., in $\left.\operatorname{Lang}\left(||^{t} \operatorname{sgn}^{t} \rtimes X(p,-)\right)\right)$ with multiplicity one. Finally, Lemma 6.1 shows that those two types do not appear in $\delta\left(||^{(p-t) / 2} \operatorname{sgn}^{t}, p+t\right) \rtimes \mathbf{1}$. This is a contradiction that proves that $\operatorname{Lang}\left(\delta\left(||^{(p-t) / 2} \operatorname{sgn}^{t}, p+t\right) \rtimes \mathbf{1}\right)$ is only non-tempered irreducible subquotient of $\delta\left(||^{(p-t) / 2} \operatorname{sgn}^{t}, p+t\right) \rtimes \mathbf{1}$. Now, Lemma 9.3 (ii) implies that other possible irreducible subquotients of $\delta\left(||^{(p-t) / 2} \operatorname{sgn}^{t}, p+t\right) \rtimes \mathbf{1}$ are discrete series $X(p, t), X(p,-t), X(t,-p)$, and $X(-t,-p)$. Lemma 6.1 shows that the types $(p+1, t+2)$ and $(-t-2,-p-1)$ do not appear in $\delta\left(||^{(p-t) / 2} \operatorname{sgn}^{t}, p+t\right) \rtimes \mathbf{1}$. Hence, $X(p, t)$ and $X(-t,-p)$ are not subquotients. We denote this fact as follows:

$$
\delta\left(||^{(p-t) / 2} \operatorname{sgn}^{t}, p+t\right) \rtimes \mathbf{1} \geq X(p, t), X(-t,-p) .
$$

Also, Lemma 6.1 shows that the pairs $(p+1,-t)$ and $(t,-p-1)$ appear $\delta\left(||^{(p-t) / 2} \operatorname{sgn}^{t}, p+t\right) \rtimes \mathbf{1}$ with multiplicity one. This means that $X(p,-t)$ and $X(t,-p)$ appear with multiplicity at most one. It remains to show that they appear with multiplicity one. Thus, in $\mathcal{R}(S p(4, \mathbb{R}))$, we have the following:

$$
\begin{aligned}
& \delta\left(||^{(p-t) / 2} \operatorname{sgn}^{t}, p+t\right) \rtimes \mathbf{1}= \\
& \quad m \cdot X(p,-t)+n \cdot X(t,-p)+\operatorname{Lang}\left(\delta\left(||^{(p-t) / 2} \operatorname{sgn}^{t}, p+t\right) \rtimes \mathbf{1}\right),
\end{aligned}
$$

where $m, n \in\{0,1\}$. Next, we consider the $U(2)$-type $(p,-t-1)$. Lemma 6.1 implies it appears with multiplicity one in $\delta\left(||^{(p-t) / 2} \operatorname{sgn}^{t}, p+t\right) \rtimes \mathbf{1}$ and the description of discrete series given by (Section 8 , (dis)) shows that this type does not appear in $X(p,-t)$ and in $X(t,-p)$. Thus, it appears in $\operatorname{Lang}\left(\delta\left(||^{(p-t) / 2} \operatorname{sgn}^{t}, p+t\right) \rtimes \mathbf{1}\right)$ (with multiplicity one). Next, the above discussion also shows that, for $w \in\{ \pm\}$, only possible non-tempered irreducible subquotient of ||$^{t} \operatorname{sgn}^{t} \rtimes X(p, w)$, except its Langlands quotient, is $\operatorname{Lang}\left(\delta\left(||^{(p-t) / 2} \operatorname{sgn}^{t}, p+t\right) \rtimes \mathbf{1}\right)$, but the type $(p,-t-1)$ do not appear in 
||$^{t} \operatorname{sgn}^{t} \rtimes X(p, w)$ by Lemma 6.1. Hence, only non-tempered irreducible subquotient of ||$^{t} \operatorname{sgn}^{t} \rtimes X(p, w)$, is its Langlands quotient. Also, using Lemma 6.1 the description of discrete series given by (Section 8, (dis)) we easily check

$$
\begin{aligned}
||^{t} \operatorname{sgn}^{t} \rtimes X(p,+)= & m_{1} \cdot X(p, t)+n_{1} \cdot X(p,-t) \\
& +\operatorname{Lang}\left(||^{t} \operatorname{sgn}^{t} \rtimes X(p,+)\right), \\
||^{t} \operatorname{sgn}^{t} \rtimes X(p,-)= & m_{2} \cdot X(t,-p)+n_{2} \cdot X(-t,-p) \\
& +\operatorname{Lang}\left(||^{t} \operatorname{sgn}^{t} \rtimes X(p,-)\right),
\end{aligned}
$$

$m_{1}, m_{2}, n_{1}, n_{2} \in\{0,1\}$, in $\mathcal{R}(S p(4, \mathbb{R}))$.

Now, since $\zeta\left(||^{(p-t) / 2} \operatorname{sgn}^{t}, p+t\right) \rtimes \mathbf{1}$ does not contain large subquotients, (9.4) shows that all large subquotients of ||$^{p} \operatorname{sgn}^{p+1} \times \mid{ }^{t} \boldsymbol{s g n} n^{t} \rtimes \mathbf{1}$ belong to $\delta\left(||(p-t) / 2 \operatorname{sgn}^{t}, p+t\right) \rtimes \mathbf{1}$. Furthermore, ||$^{t} \operatorname{sgn}^{t} \rtimes X(p, w), w \in\{ \pm\}$ contains a large subquotient. Now, (10.4) and (10.5) imply that $X(p,-t)$ and $X(t,-p)$ must be large and $m=n=n_{1}=m_{2}=1$. Now, we are ready to prove the claim (10.1).

Put $\lambda=(t,-t)$ and $\mu=(0, t-p)$, so that $\lambda+\mu=(t,-p)$. Next, acting by the elements of the Weyl group $W$ of $S p(4, \mathbb{R})$ :

$(p, t) \rightarrow(t, p) \rightarrow(t,-p)(p-t, 0) \rightarrow(0, p-t) \rightarrow(0, t-p)(t, t) \rightarrow(t, t) \rightarrow(t,-t)$,

we see that there is a positive root system of the roots of $S p(4, \mathbb{R})$ such that $\lambda$ is dominant and $\mu$ is dominant integral. This means that the functors $\varphi_{\lambda+\mu}^{\lambda}$ and $\psi_{\lambda}^{\lambda+\mu}$ are well-defined (see the beginning of Section 3). Clearly, the same is true for the Siegel Levi subgroup. Now, Lemma 3.4 (see (3.4)) implies:

$$
\psi_{\lambda}^{\lambda+\mu}\left(\delta\left(||^{(t-p) / 2} \operatorname{sgn}^{p}, p+t\right) \rtimes \mathbf{1}\right) \simeq \psi_{\lambda}^{\lambda+\mu}\left(\delta\left(||^{(t-p) / 2} \operatorname{sgn}^{p}, p+t\right)\right) \rtimes \mathbf{1} .
$$

Next, using Lemma 4.2 (ii), we see that

$$
\psi_{\lambda}^{\lambda+\mu}\left(\delta\left(||^{(t-p) / 2} \operatorname{sgn}^{p}, p+t\right)\right) \simeq \delta(\operatorname{sgn}, 2 t)=\delta(1,2 t) .
$$

Hence, by Lemma 8.1,

$$
\psi_{\lambda}^{\lambda+\mu}\left(\delta\left(||^{(p-t) / 2} \operatorname{sgn}^{t}, p+t\right) \rtimes \mathbf{1}\right) \simeq \delta(1,2 t) \rtimes 1 \simeq X^{1}(t,-t) \oplus X^{2}(t,-t) .
$$

Also, [10, Theorem 12.26] (see also (8.1)) implies

$$
\begin{aligned}
& \psi_{\lambda}^{\lambda+\mu}(X(p,-t)) \simeq X^{1}(t,-t), \\
& \psi_{\lambda}^{\lambda+\mu}(X(t,-p)) \simeq X^{2}(t,-t) .
\end{aligned}
$$

Now, Theorem 3.2 (v), implies

$$
\begin{gathered}
\operatorname{Hom}_{(\mathfrak{s p}(2, \mathbb{R}), U(2))}\left(\delta\left(||^{(t-p) / 2} \operatorname{sgn}^{p}, p+t\right) \rtimes \mathbf{1}, \varphi_{\lambda+\mu}^{\lambda}(X)\right) \\
\simeq \operatorname{Hom}_{(\mathfrak{s p}(2, \mathbb{R}), U(2))}\left(X^{1}(t,-t) \oplus X^{2}(t,-t), X\right) .
\end{gathered}
$$


Next, taking $X=X^{i}(t,-t), i=1,2$, we see that (10.8) implies that there is a non-zero equivariant-map $\delta\left(||^{(t-p) / 2} \operatorname{sgn}^{p}, p+t\right) \rtimes \mathbf{1} \rightarrow \varphi_{\lambda+\mu}^{\lambda}\left(X^{i}(t,-t)\right), i=$ 1,2 . We denote this map by $J$. We show that $X(p,-t)(\operatorname{resp} . X(t,-p))$ is isomorphic to $\operatorname{Im} J$ if $i=1$ (resp. $i=2$ ). We prove this fact for $i=1$. The proof for $i=2$ is analogous. First, any irreducible subquotient of $\varphi_{\lambda+\mu}^{\lambda}\left(X^{1}(t,-t)\right)$ maps under $\psi_{\lambda}^{\lambda+\mu}$ to a module that has in its composition series irreducible subquotients isomorphic to $X^{1}(t,-t)$. Thus, $(10.7)$ shows that $X(t,-p)$ is not a subquotient of $\operatorname{Im} J$. Next, since $\operatorname{Lang}\left(\delta\left(||^{(p-t) / 2} \operatorname{sgn}^{t}, p+t\right) \rtimes \mathbf{1}\right.$ appears in $\delta\left(||^{(t-p) / 2} \operatorname{sgn}^{p}, p+t\right) \rtimes \mathbf{1}$ with multiplicity one and a as unique irreducible subrepresentation, it is not a subquotient of $\operatorname{Im} J$ (otherwise, $J$ would be an embedding and $X(t,-p)$ would appear too). Now, (10.4) completes the proof of the claim. In particular, we have demonstrated

$$
\begin{aligned}
\operatorname{Lang} & \left(\delta\left(||^{(p-t) / 2} \operatorname{sgn}^{t}, p+t\right) \rtimes \mathbf{1}\right) \\
\hookrightarrow & \hookrightarrow \delta\left(||^{(t-p) / 2} \operatorname{sgn}^{p}, p+t\right) \rtimes \mathbf{1} \rightarrow X(p,-t) \oplus X(t,-p) .
\end{aligned}
$$

Now, taking contragredients we obtain (10.1) (see (9.6) and the discussion before the statement of Lemma 7.2).

Now, we prove (10.2). First, we show the following:

$$
\begin{aligned}
& ||^{t} \operatorname{sgn}^{t} \rtimes X(p,+) \geq X(p, t) \text { (multiplicity one), } \\
& ||^{t} \operatorname{sgn}^{t} \rtimes X(p,-) \geq X(-t,-p) \text { (multiplicity one), } \\
& ||^{t} \operatorname{sgn}^{t} \rtimes V_{p} \ngtr X(p, t), X(-t,-p) .
\end{aligned}
$$

We prove (10.9) in two steps. First, if $t=p-1$, then $X(p, t)$ (resp. $(X(-t,-p))$ contain $U(2)$-type $(p+1, p+1)$ (resp. $(-p-1,-p-1))$ with multiplicity one, but this type is not contained in ||$^{t} \operatorname{sgn}^{t} \rtimes V_{p}$ and it is contained in ||$^{t} \operatorname{sgn}^{t} \rtimes X(p,+)$ (resp. ||$\left.{ }^{t} \operatorname{sgn}^{t} \rtimes X(p,-)\right)$ with multiplicity one. Now, Lemma 9.3 (ii) completes the proof of (10.9). In general, we let $\lambda=(t, t+1)$, $\mu=(0, p-t-1)$. Hence $\lambda+\mu=(t, p)$. Clearly, the functors $\varphi_{\lambda+\mu}^{\lambda}$ and $\psi_{\lambda}^{\lambda+\mu}$ are well-defined. Lemma 3.4 implies the following $(w \in\{ \pm\})$ :

$$
\begin{aligned}
& \psi_{\lambda}^{\lambda+\mu}\left(||^{t} \operatorname{sgn}^{t} \rtimes X(p, w)\right) \simeq||^{t} \operatorname{sgn}^{t} \rtimes X(t+1, w), \\
& \psi_{\lambda}^{\lambda+\mu}\left(||^{t} \operatorname{sgn}^{t} \rtimes V_{p}\right) \simeq||^{t} \operatorname{sgn}^{t} \rtimes V_{t+1} .
\end{aligned}
$$

Since $\psi_{\lambda}^{\lambda+\mu}(X(p, t)) \simeq X(t+1, t)$ and $\psi_{\lambda}^{\lambda+\mu}(X(-t,-p)) \simeq X(-t,-t-1)$, applying Theorem 3.2 (iii) we obtain (10.9). Also, we obtain $n_{1}=m_{2}=1$ in (10.5). Now, to complete the proof of (10.2), we argue as follows. Put $\lambda=(0, p-t), \mu=(t, t)$. Hence $\lambda+\mu=(t, p)$. Clearly, the functors $\varphi_{\lambda+\mu}^{\lambda}$ and $\psi_{\lambda}^{\lambda+\mu}$ are well-defined. Lemma 3.4 implies the following $(w \in\{ \pm\})$ :

$$
\psi_{\lambda}^{\lambda+\mu}\left(||^{t} \operatorname{sgn}^{t} \rtimes X(p, w)\right) \simeq 1 \rtimes X(p-t, w) .
$$


Also, ([10], Theorem 12.26) (see also (8.1)) implies $X^{1}(p, 0)=\psi_{\lambda}^{\lambda+\mu}(X(p, t))$, $X^{2}(p, 0)=\psi_{\lambda}^{\lambda+\mu}(X(p,-t)), X^{1}(0,-p)=\psi_{\lambda}^{\lambda+\mu}(X(t,-p))$, and $X^{1}(0,-p)=$ $\psi_{\lambda}^{\lambda+\mu}(X(-t,-p))$. Now, one can apply Lemma 8.1 (i) and (10.5) (where we have $\left.n_{1}=n_{2}=m_{1}=m_{2}=1\right)$ to complete the proof of (10.2). The proof is similar to that of (10.1). One just needs to observe that the type description given in Theorem 2.4 and in Section section6 implies the following description of contragredient representations: $\widetilde{X(p,-)} \simeq X(p,+), \widetilde{X(p, t)} \simeq X(-t,-p)$, and $\widehat{X(p,-t)} \simeq X(t,-p)$.

We record the following corollary of the previous proof.

Corollary 10.2. Assume $p>t>0$. Then we have the following:

(i) ||$^{t} \operatorname{sgn}^{t} \rtimes V_{p} \nsucceq X(p, t), X(p,-t), X(t,-p), X(-t,-p)$. In particular, discrete series subquotients appear in ||$^{p} \operatorname{sgn}^{p+1} \times||{ }^{t} \operatorname{sgn}^{t} \rtimes \mathbf{1}$ with multiplicity one.

(ii) $\delta\left(||^{(p+t) / 2} \operatorname{sgn}^{t}, p-t\right) \rtimes \mathbf{1} \ngtr X(p, t), X(-t,-p)$.

(iii) $\zeta\left(||^{(p-t) / 2} \operatorname{sgn}^{t}, p+t\right) \rtimes \mathbf{1} \geq X(p,-t), X(t,-p)$.

(iv) $\zeta\left(||^{(p-t) / 2} \operatorname{sgn}^{t}, p+t\right) \rtimes \mathbf{1}, \zeta\left(||^{(p+t) / 2} \operatorname{sgn}^{t}, p-t\right) \rtimes \mathbf{1} \geq X(p, t), X(-t,-p)$ (multiplicity one).

(v) The type $(p+1, t)$ (resp., $(-t,-p-1))$ appears in Lang $\left(||^{t} \operatorname{sgn}^{t} \rtimes\right.$ $X(p,+))$ (resp., in $\left.\operatorname{Lang}\left(||^{t} \operatorname{sgn}^{t} \rtimes X(p,-)\right)\right)$ with multiplicity one. The type $(p,-t-1)$ appears with multiplicity one in the Langlands quotient $\operatorname{Lang}\left(\delta\left(||^{(p-t) / 2} \operatorname{sgn}^{t}, p+t\right) \rtimes \mathbf{1}\right)$.

Proof. We prove (i). First, since $X(p,-t)$ and $X(t,-p)$ are large by Theorem 10.1, we see that ||$^{t} \operatorname{sgn}^{t} \rtimes V_{p} \geq X(p,-t), X(t,-p)$. Next, in the proof of Theorem 10.1, we have demonstrated that ||$^{t} \operatorname{sgn}^{t} \rtimes V_{p} \nsupseteq$ $X(p, t), X(-t,-p)$. Now, the fact that discrete series appear in ||$^{p} \operatorname{sgn} n^{p+1} \times$ ||$^{t} \operatorname{sgn}^{t} \rtimes \mathbf{1}$ with multiplicity one follows from Theorem 10.1. This proves (i). To prove (ii), we apply Lemma 6.1 to see that the types $(p+1, t+2)$ and $(-t-2,-p-1)$ do not appear in $\delta\left(||^{(p+t) / 2} \operatorname{sgn}^{t}, p-t\right) \rtimes \mathbf{1}$, while they appear in $X(p, t)$ and $X(-t,-p)$ with multiplicity one, respectively. (iii) follows from (i), using Theorem 10.1 and the second equality in (9.4). The inequality $\zeta\left(||^{(p-t) / 2} \operatorname{sgn}^{t}, p+t\right) \rtimes \mathbf{1} \geq X(p, t), X(-t,-p)$ follows from the second equality in (9.4) and Theorem 10.1. The other inequality follows from the last equality in (9.4) and (ii). The claimed multiplicities follow from (i). Finally, (v) is contained in the proof of Theorem 10.1.

Finally, we prove the next theorem that completes the description of all generalized principal series appearing in (9.4).

THEOREM 10.3. Assume $p>t>0$. Then there is a representation $W$ such that the following sequence is exact:

$$
W \hookrightarrow \delta\left(||^{(p+t) / 2} \operatorname{sgn}^{t}, p-t\right) \rtimes \mathbf{1} \rightarrow \operatorname{Lang}\left(\delta\left(||^{(p+t) / 2} \operatorname{sgn}^{t}, p-t\right) \rtimes \mathbf{1}\right),
$$


and

$$
\begin{aligned}
& \delta\left(||^{(p-t) / 2} \operatorname{sgn}^{t}, p+t\right) \rtimes \mathbf{1} \hookrightarrow W \\
& \quad \rightarrow \operatorname{Lang}\left(||^{t} \operatorname{sgn}^{t} \rtimes X(p,+)\right) \oplus \operatorname{Lang}\left(||^{t} \operatorname{sgn}^{t} \rtimes X(p,-)\right) .
\end{aligned}
$$

Proof. First, we apply Lemma $7.2($ see $(7.1))$ to $\delta\left(||^{(p+t) / 2} s g n^{t}, p-t\right) \rtimes \mathbf{1}$. Since in this case in (7.1) the first operator has a simple pole, we obtain the following commutative diagram:

$$
\begin{aligned}
& \delta\left(||^{(p-t) / 2} \operatorname{sgn}^{t}, p+t\right) \rtimes \mathbf{1} \longrightarrow\left.\left|{ }^{p} \operatorname{sgn}^{p+1} \times\right|\right|^{-t} \operatorname{sgn}^{t} \rtimes \mathbf{1} \\
& \simeq \mid\left|{ }^{p} \operatorname{sgn}^{p+1} \times\right|{ }^{t} \operatorname{sgn}^{t} \rtimes \mathbf{1} \\
& 0 \downarrow\left|{ }^{t} \operatorname{sgn}^{t} \times\right|{ }^{p} \operatorname{sgn}^{p+1} \rtimes \mathbf{1} \\
& \delta\left(||^{-(p-t) / 2} \operatorname{sgn}^{t}, p+t\right) \rtimes \mathbf{1} \stackrel{j}{\longrightarrow}||{ }^{t} \operatorname{sgn}^{t} \times||^{-p} \operatorname{sgn}^{p+1} \rtimes \mathbf{1}
\end{aligned}
$$

It is clear that

$$
\begin{aligned}
& \operatorname{ker} A_{2} \simeq \delta\left(||^{(p+t) / 2} \operatorname{sgn}^{t}, p-t\right) \rtimes \mathbf{1}, \\
& \operatorname{ker} A_{3} \simeq||^{t} \operatorname{sgn}^{t} \rtimes X(p,+) \oplus||^{t} \operatorname{sgn}^{t} \rtimes X(p,-) .
\end{aligned}
$$

Now, (10.10), (i) and (ii) imply

$$
\delta\left(||^{(p-t) / 2} \operatorname{sgn}^{t}, p+t\right) \rtimes \mathbf{1} \hookrightarrow \delta\left(||^{(p+t) / 2} \operatorname{sgn}^{t}, p-t\right) \rtimes \mathbf{1} .
$$

We apply Lemma 7.3 with $\eta_{u}=s g n^{t}$. First, comparing the diagrams (10.10) and (7.7) we see that $\operatorname{Im} B_{1}(t)=\operatorname{ker} A_{2}$. Similarly, the embedding (8.9) implies that the image of $\delta\left(||^{(p+t) / 2} \operatorname{sgn}^{t}, p-t\right) \rtimes \mathbf{1}$ under (isomorphism) $B_{2}(t)$ contains ker $B_{3}(t)$. This and (7.7) implies that $\delta\left(||^{(p+t) / 2} s g n^{t}, p-t\right) \rtimes \mathbf{1}$ contains a subrepresentation $W$ such that

$$
\begin{aligned}
& \delta\left(||^{(p-t) / 2} \operatorname{sgn}^{t}, p+t\right) \rtimes \mathbf{1} \hookrightarrow W \\
& \quad \rightarrow \operatorname{Lang}\left(||^{t} \operatorname{sgn}^{t} \rtimes X(p,+)\right) \oplus \operatorname{Lang}\left(||^{t} \operatorname{sgn}^{t} \rtimes X(p,-)\right) .
\end{aligned}
$$

Moreover, $B_{3}(t)$ induces an embedding

$\delta\left(||^{(p+t) / 2} \operatorname{sgn}^{t}, p-t\right) \rtimes \mathbf{1} / \delta\left(||^{(p-t) / 2} \operatorname{sgn}^{t}, p+t\right) \rtimes 1 \hookrightarrow||^{-t} \operatorname{sgn}^{t} \times||^{p} \operatorname{sgn}^{p+1} \rtimes \mathbf{1}$.

Moreover, Theorem 10.1 and Corollary Corollary 8.1 (ii) show that this image intersects the image of $j$ (given by (7.7)) exactly in the image of $W$ under $B_{3}$. Next, if we choose the intertwining operator $B_{4}$ properly,

$$
||^{-t} \operatorname{sgn}^{t} \times||^{p} \operatorname{sgn}^{p+1} \rtimes \mathbf{1} \stackrel{B_{4}}{\longrightarrow}||^{-t} \operatorname{sgn}^{t} \times||^{-p} \operatorname{sgn}^{p+1},
$$


then the composition of $B_{4} \circ B_{3}(t) \circ B_{2}(t)$ on $\operatorname{Im} B_{1}(t)=\operatorname{ker} A_{2}$ has the image isomorphic to $\operatorname{Lang}\left(\delta\left(||^{(p+t) / 2} \operatorname{sgn}^{t}, p-t\right) \rtimes \mathbf{1}\right.$ ) (applying Lemma 7.2 to $\left.\delta\left(||^{(p+t) / 2} \operatorname{sgn}^{t}, p-t\right) \rtimes \mathbf{1}\right)$. Thus

$$
\delta\left(||^{(p+t) / 2} \operatorname{sgn}^{t}, p-t\right) \rtimes \mathbf{1} / W \simeq \operatorname{Lang}\left(\delta\left(||^{(p+t) / 2} \operatorname{sgn}^{t}, p-t\right) \rtimes \mathbf{1}\right) .
$$

Before we attack degenerate principal series for regular infinitesimal character we consider easier degenerate case.

TheOREM 10.4. Assume $p=t>0$. Then we have the following:

(i) In $\mathcal{R}(S p(4, \mathbb{R}))$, we have the following:

$$
\begin{aligned}
& ||^{p} \operatorname{sgn}^{p} \times||^{p} \operatorname{sgn}^{p+1} \rtimes \mathbf{1} \\
& \quad=X^{1}(p,-p)+X^{1}(p,-p)+\operatorname{Lang}\left(||^{p} \operatorname{sgn}^{p} \rtimes X(p,+)\right) \\
& \quad+\operatorname{Lang}\left(||^{p} \operatorname{sgn}^{p} \rtimes X(p,-)\right)+\operatorname{Lang}\left(||^{p} \operatorname{sgn}^{p} \times||^{p} \operatorname{sgn}^{p+1} \times \rtimes \mathbf{1}\right) .
\end{aligned}
$$

(ii) In $\mathcal{R}(S p(4, \mathbb{R}))$, we have the following:

$$
\begin{aligned}
& ||^{p} \operatorname{sgn}^{p} \rtimes X(p,+)=X^{1}(p,-p)+\operatorname{Lang}\left(||^{p} \operatorname{sgn}^{p} \rtimes X(p,+)\right), \\
& ||^{p} \operatorname{sgn}^{p} \rtimes X(p,-)=X^{2}(p,-p)+\operatorname{Lang}\left(||^{p} \operatorname{sgn}^{p} \rtimes X(p,-)\right), \\
& ||^{p} \operatorname{sgn}^{p} \rtimes V_{p}=\operatorname{Lang}\left(||^{p} \operatorname{sgn}^{p} \times||^{p} \operatorname{sgn}^{p+1} \rtimes \mathbf{1}\right)
\end{aligned}
$$

(it is obvious how to write composition series in this case).

(iii) We have the following exact sequence:

$$
\begin{aligned}
\operatorname{Lang} & \left(||^{p} \operatorname{sgn}^{p} \rtimes X(p,+)\right) \oplus \operatorname{Lang}\left(||^{p} \operatorname{sgn}^{p} \rtimes X(p,-)\right) \\
& \hookrightarrow \zeta\left(\operatorname{sgn}^{p}, 2 p\right) \rtimes \mathbf{1} \rightarrow \operatorname{Lang}\left(||^{p} \operatorname{sgn}^{p} \times||^{p} \operatorname{sgn}^{p+1} \times \rtimes \mathbf{1}\right)
\end{aligned}
$$

(we remind the reader that Lemma 8.1 implies $\delta\left(\operatorname{sgn}^{p}, 2 p\right) \rtimes \mathbf{1} \simeq$ $\left.X^{1}(p,-p) \oplus X^{2}(p,-p)\right)$.

Proof. First, the second equality in (9.4) implies that $\delta\left(\operatorname{sgn}^{p}, 2 p\right) \rtimes \mathbf{1}$ contains all large subquotients of ||$^{p} \operatorname{sgn}^{p} \times||^{p} \operatorname{sgn}^{p+1} \rtimes \mathbf{1}$. Also, the induced representation $\mid{ }^{p} \operatorname{sgn}^{p} \rtimes X(p, \pm)$ must contain all large subquotients. Since, by Lemma 8.1, $\delta\left(\operatorname{sgn}^{p}, 2 p\right) \rtimes \mathbf{1} \simeq X^{1}(p,-p) \oplus X^{2}(p,-p)$ we see that Lemma 5.2 implies

$$
\begin{aligned}
& ||^{p} \operatorname{sgn}^{p} \rtimes X(p,+) \geq X^{1}(p,-p) \text { (multiplicity one), } \\
& ||^{p} \operatorname{sgn}^{p} \rtimes X(p,+) \nsupseteq X^{2}(p,-p), \\
& ||^{p} \operatorname{sgn}^{p} \rtimes X(p,-) \nsupseteq X^{1}(p,-p), \\
& ||^{p} \operatorname{sgn}^{p} \rtimes X(p,-) \geq X^{2}(p,-p) \text { (multiplicity one). }
\end{aligned}
$$

This also shows that both $X^{1}(p,-p)$ and $X^{2}(p,-p)$ are large. Hence

$$
||^{p} \operatorname{sgn}^{p} \rtimes V_{p} \gtrsim X^{1}(p,-p), X^{2}(p,-p) .
$$


We apply (7.7), where $p=t$ and $\eta_{u}=s g n^{p}$. Note that $B_{1}(p)$ is holomorphic and an isomorphism (see Theorem 2.5). Next, $B_{2}(p)$ is also an isomorphism. Furthermore, Lemma 8.1 and Lemma 7.3 (iii) show that

$$
\operatorname{ker} B_{3}(p) \simeq \delta\left(\operatorname{sgn}^{p}, 2 p\right) \rtimes \mathbf{1} \simeq X^{1}(p,-p)+X^{2}(p,-p) .
$$

Now, since (7.7) implies that the composition $B_{3}(p) \circ B_{2}(p) \circ B_{1}(p) \circ i$ has the image isomorphic to $\operatorname{Lang}\left(||^{p} \operatorname{sgn}^{p} \rtimes X(p,+)\right) \oplus \operatorname{Lang}\left(||^{p} \operatorname{sgn}^{p} \rtimes X(p,-)\right)$, using (10.14) we obtain

$$
\begin{aligned}
& ||^{p} \operatorname{sgn}^{p} \rtimes X(p,+)=X^{1}(p,-p)+\operatorname{Lang}\left(||^{p} \operatorname{sgn}^{p} \rtimes X(p,+)\right), \\
& ||^{p} \operatorname{sgn}^{p} \rtimes X(p,-)=X^{2}(p,-p)+\operatorname{Lang}\left(||^{p} \operatorname{sgn}^{p} \rtimes X(p,-)\right) .
\end{aligned}
$$

This proves the first two equalities in (ii). Next, (10.15) and the last equality in (9.4), using Lemma 9.2 (iii) and Lemma 9.3 (ii), show that (i) holds up to the multiplicity of the third and the fourth representation in the expansion. Next, using Lemma 6.1 , we see that a type $(p+1, p)$ (resp., $(-p,-p-1)$ ) appears in ||$^{p} \operatorname{sgn}^{p} \rtimes X(p,+)$ (resp., in ||$\left.^{p} \operatorname{sgn}^{p} \rtimes X(p,-)\right)$ with multiplicity one. Using, the description of the limits of discrete series (see Section 8), we see that $(p+1, p)$ (resp., $(-p,-p-1))$ does not appear in $X^{1}(p,-p)$ (resp., in $\left.X^{2}(p,-p)\right)$. Hence $(10.16)$ shows that a type $(p+1, p)$ (resp., $\left.(-p,-p-1)\right)$ appears in $\operatorname{Lang}\left(||^{p} \operatorname{sgn}^{p} \rtimes X(p,+)\right)$ (resp., in $\left.\operatorname{Lang}\left(||^{p} \operatorname{sgn}^{p} \rtimes X(p,-)\right)\right)$ with multiplicity one. Finally, by Lemma 6.1, they do not appear in ||$^{p} \operatorname{sgn}^{p} \rtimes V_{p}$. Hence, we obtain (i) and

$$
||^{p} \operatorname{sgn}^{p} \rtimes V_{p}=\operatorname{Lang}\left(||^{p} \operatorname{sgn}^{p} \times||^{p} \operatorname{sgn}^{p+1} \rtimes \mathbf{1}\right) .
$$

Hence (ii). The semi-simplification of the exact sequence in (iii) follows from (i) and $\delta\left(\operatorname{sgn}^{p}, 2 p\right) \rtimes \mathbf{1} \simeq X^{1}(p,-p) \oplus X^{2}(p,-p)$. The claim about the quotient is obvious. We need to show

$$
\operatorname{Lang}\left(||^{p} \operatorname{sgn}^{p} \rtimes X(p,+)\right) \oplus \operatorname{Lang}\left(||^{p} \operatorname{sgn}^{p} \rtimes X(p,-)\right) \hookrightarrow \zeta\left(\operatorname{sgn}^{p}, 2 p\right) \rtimes \mathbf{1},
$$

and this will prove (iii). Again, this follows from (5.7) since, as we noticed above, we have

$$
\begin{aligned}
& B_{3}(p) \circ B_{2}(p) \circ B_{1}(p) \circ i \\
& \quad \simeq \operatorname{Lang}\left(||^{p} s g n^{p} \rtimes X(p,+)\right) \oplus \operatorname{Lang}\left(||^{p} \operatorname{sgn}^{p} \rtimes X(p,-)\right), \\
& \operatorname{ker} B_{3}(p) \subset \operatorname{Im} B_{2}(p) \circ B_{1}(p) \circ i, \\
& \operatorname{Im} B_{3}(p) \simeq \zeta\left(\operatorname{sgn}^{p}, 2 p\right) \rtimes \mathbf{1} .
\end{aligned}
$$

The next corollary will be needed in the proof of Theorem 10.6 below.

Corollary 10.5. Assume $p>t>0$. The multiplicities of the representations $\operatorname{Lang}\left(||^{t} \operatorname{sgn}^{t} \rtimes X(p,+)\right)$ and $\operatorname{Lang}\left(||^{t} \operatorname{sgn}^{t} \rtimes X(p,-)\right)$ in $\left.\left|{ }^{t} \operatorname{sgn}^{t} \times\right|\right|^{p} \operatorname{sgn}^{p+1} \rtimes \mathbf{1}$ are exactly one. 
Proof. Put $\mu=(0, p-t)$ and $\lambda=(t, t)$. Then $\lambda+\mu=(t, p)$. Clearly, the functor $\psi_{\lambda}^{\lambda+\mu}$ is well-defined. Lemma 3.4 implies the following $(w \in\{ \pm\})$ :

$$
\begin{aligned}
& \psi_{\lambda}^{\lambda+\mu}\left(||^{t} \operatorname{sgn}^{t} \rtimes X(p, w)\right) \simeq||^{t} \operatorname{sgn}^{t} \rtimes X(t, w), \\
& \psi_{\lambda}^{\lambda+\mu}\left(||^{t} \operatorname{sgn}^{t} \rtimes V_{p}\right) \simeq||^{t} \operatorname{sgn}^{t} \rtimes V_{t} .
\end{aligned}
$$

Now, starting from the exact sequence (see Theorem 10.1 (see (10.2)):

$$
X(p, t) \oplus X(p,-t) \hookrightarrow||^{t} \operatorname{sgn}^{t} \rtimes X(p,+) \rightarrow \operatorname{Lang}\left(||^{t} \operatorname{sgn}^{t} \rtimes X(t,+)\right),
$$

and applying the exactness of the functor $\psi_{\lambda}^{\lambda+\mu}$, we obtain

$$
\begin{aligned}
\psi_{\lambda}^{\lambda+\mu}(X(p, t)) \oplus \psi_{\lambda}^{\lambda+\mu}(X(p,-t)) & \hookrightarrow \psi_{\lambda}^{\lambda+\mu}\left(||^{t} \operatorname{sgn}^{t} \rtimes X(p,+)\right) \\
& \rightarrow \psi_{\lambda}^{\lambda+\mu}\left(\operatorname{Lang}\left(||^{t} \operatorname{sgn}^{t} \rtimes X(p,+)\right)\right) .
\end{aligned}
$$

Now, since $\psi_{\lambda}^{\lambda+\mu}(X(p, t))=0$ and $\psi_{\lambda}^{\lambda+\mu}(X(p,-t))=X^{1}(t,-t)$, Theorem 10.4 (ii) (or just Theorem 3.2 (iv)) implies the isomorphism (10.18) when $w=+$ :

$$
\psi_{\lambda}^{\lambda+\mu}\left(\operatorname{Lang}\left(||^{t} \operatorname{sgn}^{t} \rtimes X(p, w)\right)\right) \simeq \operatorname{Lang}\left(||^{t} \operatorname{sgn}^{t} \rtimes X(t, w)\right),
$$

$w \in\{ \pm\}$. The other one is proved similarly. Next, Theorem 10.4 (ii) shows

$$
||^{t} \operatorname{sgn}^{t} \rtimes V_{t} \ngtr \operatorname{Lang}\left(||^{t} \operatorname{sgn}^{t} \rtimes X(t, w)\right) .
$$

Now, combining (10.17) and (10.18), we obtain

$$
||^{t} \operatorname{sgn}^{t} \rtimes V_{p} \ngtr \operatorname{Lang}\left(||^{t} \operatorname{sgn}^{t} \rtimes X(p, w)\right) .
$$

Finally, the last equality in (9.4) and Theorem 10.1 (see (10.2)) completes the proof of the corollary.

The next theorem completes the investigation of the structure of the representation ||$^{p} \operatorname{sgn}^{p+1} \times \mid{ }^{t} \operatorname{sgn}^{t} \rtimes \mathbf{1}(p>t>0)$.

TheOREM 10.6. Assume $p>t>0$. Then, we have the following:

(i) In $\mathcal{R}(S p(4, \mathbb{R}))$, we have the following:

$$
\begin{aligned}
&||{ }^{p} \operatorname{sgn}^{p+1} \times||^{t} \operatorname{sgn}^{t} \rtimes \mathbf{1} \\
&= X(p, t)+X(p,-t)+X(t,-p)+X(-t,-p)+\operatorname{Lang}\left(||^{t} \operatorname{sgn}^{t} \rtimes X(p,+)\right) \\
& \quad+\operatorname{Lang}\left(||^{t} \operatorname{sgn}^{t} \rtimes X(p,-)\right)+\operatorname{Lang}\left(\delta\left(||^{(p-t) / 2} \operatorname{sgn}^{t}, p+t\right) \rtimes \mathbf{1}\right) \\
& \quad+\operatorname{Lang}\left(\delta\left(||^{(p+t) / 2} \operatorname{sgn}^{t}, p-t\right) \rtimes \mathbf{1}\right)+\operatorname{Lang}\left(||^{t} \operatorname{sgn}^{t} \times||^{p} \operatorname{sgn}^{p+1} \rtimes \mathbf{1}\right) .
\end{aligned}
$$

(ii) We have the following exact sequence:

$$
\begin{aligned}
X(p, t) \oplus X(-t,-p) & \hookrightarrow \zeta\left(||^{(p+t) / 2} \operatorname{sgn}^{t}, p-t\right) \rtimes \mathbf{1} \\
& \rightarrow \operatorname{Lang}\left(||^{p} \operatorname{sgn}^{p+1} \times||^{t} \operatorname{sgn}^{t} \rtimes \mathbf{1}\right) .
\end{aligned}
$$


(iii) There is a representation $W^{\prime}$ such that we have the following exact sequences:

$$
\begin{gathered}
\operatorname{Lang}\left(||^{t} \operatorname{sgn}^{t} \rtimes X(p,+)\right) \oplus \operatorname{Lang}\left(||^{t} \operatorname{sgn}^{t} \rtimes X(p,-)\right) \\
\hookrightarrow W^{\prime} \rightarrow \operatorname{Lang}\left(\delta\left(||^{(p+t) / 2} \operatorname{sgn}^{t}, p-t\right) \rtimes \mathbf{1}\right)
\end{gathered}
$$

and

$$
W^{\prime} \hookrightarrow \zeta\left(||^{(p-t) / 2} \operatorname{sgn}^{t}, p+t\right) \rtimes \mathbf{1} \rightarrow \zeta\left(||^{(p+t) / 2} \operatorname{sgn}^{t}, p-t\right) \rtimes \mathbf{1} .
$$

(iv) We have the following exact sequence:

$$
\begin{gathered}
\operatorname{Lang}\left(\left.||\right|^{p} \operatorname{sgn}^{p+1} \times||^{t} \operatorname{sgn}^{t} \rtimes \mathbf{1}\right) \oplus \operatorname{Lang}\left(\delta\left(||^{(p-t) / 2} \operatorname{sgn}^{t}, p+t\right) \rtimes \mathbf{1}\right) \\
\hookrightarrow||^{t} \operatorname{sgn}^{t} \rtimes V_{p} \rightarrow \operatorname{Lang}\left(\delta\left(||^{(p+t) / 2} \operatorname{sgn}^{t}, p-t\right) \rtimes \mathbf{1}\right) .
\end{gathered}
$$

Proof. We use the notation and results established in the proof of Theorem 10.3. We also consider some (induced) non-zero intertwining operator:

$$
||^{-t} \operatorname{sgn}^{t} \times||^{-p} \operatorname{sgn}^{p+1} \rtimes \mathbf{1} \stackrel{B_{5}}{\longrightarrow}||^{-p} \operatorname{sgn}^{p+1} \times||^{-t} \operatorname{sgn}^{t} \rtimes \mathbf{1} .
$$

Now, we note that the composition $B_{5} \circ B_{4} \circ B_{3}(t) \circ B_{2}(t)$ has the image

$$
\begin{gathered}
B_{5} \circ B_{4} \circ B_{3}(t) \circ B_{2}(t)\left(||^{p} \operatorname{sgn}^{p+1} \times||^{t} \operatorname{sgn}^{t} \rtimes \mathbf{1}\right) \\
\simeq \operatorname{Lang}\left(||^{p} \operatorname{sgn}^{p+1} \times||^{t} \operatorname{sgn}^{t} \rtimes \mathbf{1}\right) .
\end{gathered}
$$

Also, we see that Lemma 7.3 (iii) and (iv) imply that the semisimplifications in $\mathcal{R}\left(S p(4, \mathbb{R})\right.$ ) of ker $B_{2}(t)$ (resp., ker $\left.B_{3}(t)\right)$ are $\{0\}$ (resp., described by Theorem 10.1 (see $(10.1))$ ). Next, $\operatorname{ker} B_{4}(t)$ (see (8.11)) has the same semisimplification in $\mathcal{R}(S p(4, \mathbb{R}))$ as $\operatorname{ker} B_{2}(t)$ and $\operatorname{ker} B_{5}(t)$ (see $\left.(10.22)\right)$ is isomorphic to $\delta\left(||^{-(p+t) / 2} \operatorname{sgn}^{p+1}, p-t\right) \rtimes \mathbf{1}$, which has the same semisimplification in $\mathcal{R}(S p(4, \mathbb{R}))$ as $\delta\left(||^{(p+t) / 2} \operatorname{sgn}^{t}, p-t\right) \rtimes \mathbf{1}$, described by Theorem 10.3. We conclude that, except the Langlands quotient, ||$^{p} \operatorname{sgn}^{p+1} \times||{ }^{t} \operatorname{sgn}^{t} \rtimes \mathbf{1}$ can have only the following irreducible subquotients: $X(p, t), X(p,-t)$, $X(t,-p), X(-t,-p), \operatorname{Lang}\left(||{ }^{t} \operatorname{sgn}^{t} \rtimes X(p,+)\right), \operatorname{Lang}\left(||{ }^{t} \operatorname{sgn}^{t} \rtimes X(p,-)\right)$, $\operatorname{Lang}\left(\delta\left(||^{(p-t) / 2} \operatorname{sgn}^{t}, p+t\right) \rtimes \mathbf{1}\right)$, and $\operatorname{Lang}\left(\delta\left(||^{(p+t) / 2} \operatorname{sgn}^{t}, p-t\right) \rtimes \mathbf{1}\right)$ up to multiplicity. It remains to determine multiplicities.

First, by Corollary 10.2 (i) all discrete series appear with multiplicity one. Next, by Corollary 10.5, Lang $\left(||^{t} \operatorname{sgn}^{t} \rtimes X(p, \pm)\right)$ also appear with multiplicity one. Obviously, $\operatorname{Lang}\left(||^{p} \operatorname{sgn}^{p+1} \times||^{t} \operatorname{sgn}^{t} \rtimes \mathbf{1}\right)$ appears with multiplicity one too. Next, if we consider how the images under the intertwining operators $B_{2}(t), B_{3}(t) \circ B_{2}(t)$, and $B_{4} \circ B_{3}(t) \circ B_{2}(t)$ intersect the kernels ker $B_{3}(t)$, ker $B_{4}$, and $\operatorname{ker} B_{5}$, respectively, we conclude that $\operatorname{Lang}\left(\delta\left(||^{(p+t) / 2} \operatorname{sgn}^{t}, p-\right.\right.$ t) $\rtimes \mathbf{1}$ ) appears with multiplicity one.

It remains to determine the multiplicity of $\operatorname{Lang}\left(\delta\left(||^{(p-t) / 2} \operatorname{sgn}^{t}, p+t\right) \rtimes \mathbf{1}\right)$. We consider the two cases:

Assume $p \equiv t+1(\bmod 2)$. Then the multiplicity of type $(p,-p)$ in $\delta\left(||^{(p-t) / 2} \operatorname{sgn}^{t}, p+t\right) \rtimes \mathbf{1}, \delta\left(||^{(p+t) / 2} \operatorname{sgn}^{t}, p-t\right) \rtimes \mathbf{1},\left|{ }^{t} \operatorname{sgn}^{t} \times\right| \mid{ }^{p} \operatorname{sgn}^{p+1} \rtimes \mathbf{1}$, 
using Lemma 6.1 , is $(p-t+1) / 2,(p+t+1) / 2$, and $p$, respectively. Next, using the description of the types of discrete series (given in Section 8) we see that $X(p,-t)$ and $X(t,-p)$ do not contain that type. Hence, Theorem 10.1 (see (10.1)) implies that its multiplicity in $\operatorname{Lang}\left(\delta\left(||^{(p-t) / 2} \operatorname{sgn}^{t}, p+t\right) \rtimes \mathbf{1}\right)$ must be $(p-t+1) / 2$. Since

$$
(p-t+1) / 2+(p+t+1) / 2=p+1>p,
$$

we see that (9.4) implies that $\operatorname{Lang}\left(\delta\left(||^{(p-t) / 2} \operatorname{sgn}^{t}, p+t\right) \rtimes \mathbf{1}\right)$ is not a subquotient of $\zeta\left(||^{(p-t) / 2} \operatorname{sgn}^{t}, p+t\right) \rtimes 1$. This proves (i) when $p \equiv t+1(\bmod 2)$.

Assume $p \equiv t(\bmod 2)$. Choose $(m, n) \in \mathbb{Z}^{2}$ such that $m>n>0$ and $m \equiv n+1(\bmod 2)$. Then, by Lemma 3.1 ,

$$
\psi_{(p, t)}^{(p+m, t+n)}\left(||^{p+m} \operatorname{sgn}^{p+m+1} \times||^{t+n} \operatorname{sgn}^{t+n} \rtimes \mathbf{1}\right) \simeq||^{p} \operatorname{sgn}^{p+1} \times||^{t} \operatorname{sgn}^{t} \rtimes \mathbf{1} .
$$

Since by the previous case ||$^{p+m} s g n^{p+m+1} \times||^{t+n} \operatorname{sgn}^{t+n} \rtimes \mathbf{1}$ is multiplicity free we see that ||$^{p} s g n^{p+1} \times||^{t} \operatorname{sgn}^{t} \rtimes \mathbf{1}$ is also multiplicity free. This completes the proof of (i).

In order to prove (ii), we note that the semisimplification of (10.19) follows from (i), Theorem 10.3 and (9.4). To show the exact sequence in (10.19), we remind the reader that we use the notation from the proof of Theorem 10.3. In particular, we have

$$
\begin{aligned}
& \operatorname{ker} B_{1}(t) \simeq \zeta\left(||^{(t+p) / 2} \operatorname{sgn}^{t}, p-t\right) \rtimes \mathbf{1}, \\
& \operatorname{Im} B_{1}(t) \simeq \delta\left(||^{(t+p) / 2} \operatorname{sgn}^{t}, p-t\right) \rtimes \mathbf{1} .
\end{aligned}
$$

Now, as (7.7) shows, we have

$$
\operatorname{Im} i \simeq||^{t} \operatorname{sgn}^{t} \rtimes X(p,+) \oplus||^{t} \operatorname{sgn}^{t} \rtimes X(p,-) .
$$

Thus, Theorem 10.1 (see (10.2)) and Theorem 10.3 show

$$
\operatorname{ker} B_{1}(t) \cap \operatorname{Im} i \simeq X(p, t) \oplus X(-t,-p) \text {. }
$$

Also, inducing in stages and using Theorem 2.5, we obtain

$$
||^{p} \operatorname{sgn}^{p+1} \times||^{t} \operatorname{sgn}^{t} \rtimes \mathbf{1} \rightarrow \zeta\left(||^{(t+p) / 2} \operatorname{sgn}^{t}, p-t\right) \rtimes \mathbf{1} .
$$

Since $\operatorname{Lang}\left(||^{p} \operatorname{sgn}^{p+1} \times||^{t} \operatorname{sgn}^{t} \rtimes \mathbf{1}\right)$ is a unique irreducible quotient of ||$^{p} \operatorname{sgn}^{p+1} \times \mid{ }^{t} \operatorname{sgn}^{t} \rtimes \mathbf{1}$, we obtain (10.19). This proves (ii). (iii) follows directly from the fact that the isomorphism $B_{2}(t)$ induces the embedding (10.11), Theorems 2.5 and 10.3. In fact,

$$
W^{\prime} \simeq W /\left(\delta\left(||^{(p-t) / 2} \operatorname{sgn}^{t}, p+t\right) \rtimes \mathbf{1}\right) .
$$

Finally, we prove (iv). First, the semisimplification of (iv) holds (using the last equality in (9.4), Theorems 10.1 and 10.3, and (i)). Next, inducing in stages and applying Theorem 2.3, we obtain (see (7.7))

$$
\operatorname{Im} i \hookrightarrow||^{t} \operatorname{sgn}^{t} \times||^{p} \operatorname{sgn}^{p+1} \rtimes \mathbf{1} \rightarrow||^{t} \operatorname{sgn}^{t} \times V_{p} .
$$


Hence, (10.23) and (10.19) implies

$$
\operatorname{Lang}\left(||^{p} \operatorname{sgn}^{p+1} \times||^{t} \operatorname{sgn}^{t} \rtimes \mathbf{1}\right) \hookrightarrow||^{t} \operatorname{sgn}^{t} \times V_{p} .
$$

Similarly, using the inclusions

$$
\delta\left(||^{-(p-t) / 2} \operatorname{sgn}^{t}, p+t\right) \rtimes \mathbf{1},||^{t} \operatorname{sgn}^{t} \times V_{p} \hookrightarrow||^{t} \operatorname{sgn}^{t} \times||^{-p} \operatorname{sgn}^{p+1} \rtimes \mathbf{1},
$$

that follow applying Theorems 2.4 and 2.5, we see that the images of the left-hand sides must intersect. Hence

$$
\operatorname{Lang}\left(\delta\left(||^{(p-t) / 2} \operatorname{sgn}^{t}, p+t\right) \rtimes \mathbf{1} \hookrightarrow||^{t} \operatorname{sgn}^{t} \times V_{p}\right.
$$

using (i) and Theorem 10.1 (see (10.2)). Now, (iv) follows.

We end this section considering one more degenerate case that completes the investigation of ||$^{p} \operatorname{sgn}^{p+1} \times||^{t} \operatorname{sgn}^{t} \rtimes \mathbf{1}$ under the assumption (9.2).

ThEOREM 10.7. Assume $p>t=0$. Then we have the following:

(i) In $\mathcal{R}(S p(4, \mathbb{R}))$, we have the following:

$\mid{ }^{p} \operatorname{sgn}^{p+1} \times 1 \rtimes \mathbf{1}=X^{1}(p, 0)+X^{2}(p, 0)+X^{1}(0,-p)+X^{2}(0,-p)$

$$
+\operatorname{Lang}\left(\delta\left(||^{p / 2}, p\right) \rtimes \mathbf{1}\right)+\operatorname{Lang}\left(||^{p} \operatorname{sgn}^{p+1} \times 1 \rtimes \mathbf{1}\right) \text {. }
$$

(ii) We have the following exact sequences:

$$
\begin{gathered}
X^{2}(p, 0) \oplus X^{1}(0,-p) \hookrightarrow \delta\left(||^{p / 2}, p\right) \rtimes \mathbf{1} \rightarrow \operatorname{Lang}\left(\delta\left(||^{p / 2}, p\right) \rtimes \mathbf{1}\right), \\
X^{1}(p, 0) \oplus X^{2}(0,-p) \hookrightarrow \zeta\left(||^{p / 2}, p\right) \rtimes \mathbf{1} \rightarrow \operatorname{Lang}\left(||^{p} \operatorname{sgn}^{p+1} \times 1 \rtimes \mathbf{1}\right),
\end{gathered}
$$

Proof. We use the translation functors. Let $\left(m_{1}, m_{2}\right) \in \mathbb{Z}^{2}$ be any pair such that $m_{1}>m_{2}>0$. Put $\mu=\left(m_{1},-m_{2}\right), \lambda=(p, 0)$. Hence $\lambda+\mu=\left(p+m_{1},-m_{2}\right)$. We see that $\psi_{\lambda}^{\lambda+\mu}$ is well defined. Next, we have the following (see Lemma 4.2):

$$
\psi_{\lambda}^{\lambda+\mu}\left(\delta\left(||^{\left(p+m_{1}-m_{2}\right) / 2} \operatorname{sgn}^{m_{2}}, p+m_{1}+m_{2}\right) \rtimes \mathbf{1}\right) \simeq \delta\left(||^{p / 2}, p\right) \rtimes \mathbf{1} .
$$

Now, applying Theorem 10.1 (see (10.1)), (limit-dis) in Section 8, Theorem 3.2 (iv), and the exactness of the functor $\psi_{\lambda}^{\lambda+\mu}$ we obtain (10.25) and $(10.29)$ $\psi_{\lambda}^{\lambda+\mu}\left(\operatorname{Lang}\left(\delta\left(||^{\left(p+m_{1}-m_{2}\right) / 2} \operatorname{sgn}^{m_{2}}, p+m_{1}+m_{2}\right) \rtimes \mathbf{1}\right)\right) \simeq \operatorname{Lang}\left(\delta\left(||^{p / 2}, p\right) \rtimes \mathbf{1}\right)$.

We leave details to the reader. Also, Lemma 4.2 implies (10.30)

$$
\begin{aligned}
& \psi_{(p, 0)}^{\left(p+m_{1}, m_{2}\right)}\left(\delta\left(||^{\left(p+m_{1}+m_{2}\right) / 2} \operatorname{sgn}^{m_{2}}, p+m_{1}-m_{2}\right) \rtimes \mathbf{1}\right) \simeq \delta\left(||^{p / 2}, p\right) \rtimes \mathbf{1}, \\
& \psi_{(p, 0)}^{\left(p+m_{1}, m_{2}\right)}\left(\zeta\left(||^{\left(p+m_{1}+m_{2}\right) / 2} \operatorname{sgn}^{m_{2}}, p+m_{1}-m_{2}\right) \rtimes \mathbf{1}\right) \simeq \zeta\left(||^{p / 2}, p\right) \rtimes \mathbf{1},
\end{aligned}
$$


Next, Remark 3.1 implies that the functors $\psi_{\lambda}^{\lambda+\mu}$ and $\psi_{(p, 0)}^{\left(p+m_{1}, m_{2}\right)}$ are naturally isomorphic. Hence, (10.28), (10.29), and Theorem 10.3, implies

$(10.31) \psi_{(p, 0)}^{\left(p+m_{1}, m_{2}\right)}\left(\operatorname{Lang}\left(\delta\left(||^{\left(p+m_{1}+m_{2}\right) / 2} \operatorname{sgn}^{m_{2}}, p+m_{1}-m_{2}\right) \rtimes \mathbf{1}\right)\right)=0$.

Also, (10.19), the second formula in (10.30), (limit-dis) in Section 8, Theorem 3.2 (iv), and the exactness of the functor $\psi_{\lambda}^{\lambda+\mu}$ imply (10.26) and

$$
\begin{aligned}
& \psi_{(p, 0)}^{\left(p+m_{1}, m_{2}\right)}\left(\operatorname{Lang}\left(||^{p+m_{1}} \operatorname{sgn}^{p+m_{1}+1} \times||^{m_{2}} \operatorname{sgn}^{m_{2}} \rtimes \mathbf{1}\right)\right) \\
& \simeq \operatorname{Lang}\left(||^{p} \operatorname{sgn}^{p+1} \times 1 \rtimes \mathbf{1}\right) .
\end{aligned}
$$

Now, since we have proved (10.25) and (10.26), we obtain (i) applying the first equality in (9.4). It remains to prove (10.27). Clearly, the functor $\psi_{(0, p)}^{\left(m_{2}, p+m_{1}\right)}$ is well defined and, by Remark 3.1, naturally isomorphic to $\psi_{\lambda}^{\lambda+\mu}$ and $\psi_{(p, 0)}^{\left(p+m_{1}, m_{2}\right)}$. Also, Lemma 3.4 implies

$$
\psi_{(0, p)}^{\left(m_{2}, p+m_{1}\right)}\left(||^{m_{2}} \operatorname{sgn}^{m_{2}} \rtimes V_{p+m_{1}}\right) \simeq 1 \rtimes V_{p} .
$$

Now, Theorem 10.6 (iv) and the exactness of the functor $\psi_{(0, p)}^{\left(m_{2}, p+m_{1}\right)}$, implies (10.27), applying (10.29), (10.31) and (10.32).

11. COMPOSITION SERIES IN THE CASE OF INTEGRAL INFINITESIMAL CHARACTER III

In this section we consider $\left|{ }^{p} \operatorname{sgn}^{p} \times\right|{ }^{t} \operatorname{sgn}^{t+1} \rtimes \mathbf{1}$. We assume that (9.2) holds. This is the last case among those described in (9.1).

TheOREM 11.1. Assume $p>t>0$. Then we have the following:

(i) We have the following exact sequence:

$$
\begin{aligned}
\operatorname{Lang}\left(\delta\left(||^{(p+t) / 2} \operatorname{sgn}^{t+1}, p-t\right) \rtimes \mathbf{1}\right) & \hookrightarrow||^{p} \operatorname{sgn}^{p} \rtimes V_{t} \\
& \rightarrow \operatorname{Lang}\left(||^{p} \operatorname{sgn}^{p} \times||^{t} \operatorname{sgn}^{t+1} \rtimes \mathbf{1}\right) .
\end{aligned}
$$

(ii) ||$^{p} \operatorname{sgn}^{p} \rtimes X(t,+)$ has the following filtration $V_{0} \subset V_{1} \subset V_{2} \simeq||^{p} \operatorname{sgn}^{p} \rtimes$ $X(t,+)$, where

$$
\begin{aligned}
& V_{0} \simeq X(p,-t), \\
& V_{1} / V_{0} \text { has a filtration consisting of } \\
& \operatorname{Lang}\left(\delta\left(||^{(p-t) / 2} \operatorname{sgn}^{t}, p+t\right) \rtimes \mathbf{1}\right), \operatorname{Lang}\left(||^{t} \operatorname{sgn}^{t} \rtimes X(p,+)\right), \\
& V_{2} / V_{1} \simeq \operatorname{Lang}\left(||^{p} \operatorname{sgn}^{p} \rtimes X(t,+)\right) \text {. }
\end{aligned}
$$


(iii) ||${ }^{p} \operatorname{sgn}^{p} \rtimes X(t,-)$ has the following filtration $V_{0} \subset V_{1} \subset V_{2} \simeq||{ }^{p} \operatorname{sgn}^{p} \rtimes$ $X(t,+)$, where

$V_{0} \simeq X(t,-p)$,

$V_{1} / V_{0}$ has a filtration consisting of

$\operatorname{Lang}\left(\delta\left(||^{(p-t) / 2} \operatorname{sgn}^{t}, p+t\right) \rtimes \mathbf{1}\right), \operatorname{Lang}\left(||^{t} \operatorname{sgn}^{t} \rtimes X(p,-)\right)$,

$V_{2} / V_{1} \simeq \operatorname{Lang}\left(||^{p} \operatorname{sgn}^{p} \rtimes X(t,-)\right)$.

(iv) $\zeta\left(||^{(p+t) / 2} \operatorname{sgn}^{t+1}, p-t\right) \rtimes \mathbf{1}$ has the following filtration $V_{0} \subset V_{1} \subset V_{2} \simeq$ $\zeta\left(||^{(p+t) / 2} \operatorname{sgn}^{t+1}, p-t\right) \rtimes \mathbf{1}$, where

$V_{0} \simeq \operatorname{Lang}\left(\delta\left(||^{(p-t) / 2} \operatorname{sgn}^{t}, p+t\right) \rtimes \mathbf{1}\right)$,

$V_{1} / V_{0} \simeq \operatorname{Lang}\left(||^{p} \operatorname{sgn}^{p} \rtimes X(t,+)\right) \oplus \operatorname{Lang}\left(||^{p} \operatorname{sgn}^{p} \rtimes X(t,-)\right)$,

$V_{2} / V_{1} \simeq \operatorname{Lang}\left(||^{p} \operatorname{sgn}^{p} \times||^{t} \operatorname{sgn}^{t+1} \rtimes \mathbf{1}\right)$.

(v) $\zeta\left(||^{(p-t) / 2} \operatorname{sgn}^{t+1}, p+t\right) \rtimes \mathbf{1}$ has the following filtration $V_{0} \subset V_{1} \subset V_{2} \subset$ $V_{3} \subset V_{4} \simeq \zeta\left(||^{(p-t) / 2} \operatorname{sgn}^{t+1}, p+t\right) \rtimes \mathbf{1}$, where

$V_{0} \simeq \operatorname{Lang}\left(\delta\left(||^{(p-t) / 2} \operatorname{sgn}^{t}, p+t\right) \rtimes \mathbf{1}\right.$,

$V_{1} / V_{0} \simeq \operatorname{Lang}\left(||^{p} \operatorname{sgn}^{p} \rtimes X(t,+)\right) \oplus \operatorname{Lang}\left(||^{p} \operatorname{sgn}^{p} \rtimes X(t,-)\right)$,

$V_{2} / V_{1} \simeq \operatorname{Lang}\left(||^{p} \operatorname{sgn}^{p} \times||^{t} \operatorname{sgn}^{t+1} \rtimes \mathbf{1}\right)$,

$V_{3} / V_{2} \simeq \operatorname{Lang}\left(||^{t} \operatorname{sgn}^{t} \rtimes X(p,+)\right) \oplus \operatorname{Lang}\left(||^{t} \operatorname{sgn}^{t} \rtimes X(p,-)\right)$,

$V_{4} / V_{3} \simeq \operatorname{Lang}\left(\delta\left(||^{(p+t) / 2} \operatorname{sgn}^{t}, p-t\right) \rtimes \mathbf{1}\right)$.

Proof. First, we write appropriate analogue of the diagram (7.7). We have the following:

$$
\begin{aligned}
& \oplus_{\varepsilon \in\{ \pm\}}||^{p} \operatorname{sgn}^{p} \rtimes X(t, \varepsilon) \longrightarrow i \quad\left|{ }^{p} \operatorname{sgn}^{p} \times\right| \mid{ }^{t} \operatorname{sgn}^{t+1} \rtimes \mathbf{1} \\
& B_{1}(p) \downarrow \\
& ||^{t} \operatorname{sgn}^{t+1} \times||^{p} \operatorname{sgn}^{p} \rtimes \mathbf{1} \\
& B(p) \downarrow \quad B_{2}(p) \simeq \downarrow \\
& \left.\left|{ }^{t} \operatorname{sgn}^{t+1} \times\right|\right|^{-p} \operatorname{sgn}^{p} \rtimes \mathbf{1} \\
& B_{3}(p) \downarrow \\
& \oplus_{\varepsilon \in\{ \pm\}}||^{-p} \operatorname{sgn}^{p} \rtimes X(t, \varepsilon) \stackrel{j}{\longrightarrow}||^{-p} \operatorname{sgn}^{p} \times||^{t} \operatorname{sgn}^{t+1} \rtimes \mathbf{1} \\
& B_{4} \downarrow \\
& ||^{-p} \operatorname{sgn}^{p} \times||^{-t} \operatorname{sgn}^{t+1} \rtimes \mathbf{1} \text {. }
\end{aligned}
$$


Actually, we have added the last arrow to the diagram (11.1), where $B_{4}$ is some non-zero operator induced form that of ||$^{t} \operatorname{sgn}^{t+1} \rtimes \mathbf{1} \rightarrow||^{-t} \operatorname{sgn}^{t+1} \rtimes \mathbf{1}$. It has kernel equal to the image of $j$. Also, we recall that $B_{1}(p)$ is holomorphic (see Lemma 7.3). Also, we recall that

$$
\begin{aligned}
& \operatorname{ker} B_{1}(p) \simeq \delta\left(||^{(p+t) / 2} \operatorname{sgn}^{t+1}, p-t\right) \rtimes \mathbf{1}=\delta\left(||^{(p+t) / 2} \operatorname{sgn}^{t}, p-t\right) \rtimes \mathbf{1}, \\
& \operatorname{Im} B_{1}(p) \simeq \zeta\left(||^{(p+t) / 2} \operatorname{sgn}^{t+1}, p-t\right) \rtimes \mathbf{1}
\end{aligned}
$$

and

$$
\begin{aligned}
& \operatorname{ker} B_{3}(p) \simeq \delta\left(||^{(t-p) / 2} \operatorname{sgn}^{p}, p+t\right) \rtimes \mathbf{1}=\delta\left(||^{(t-p) / 2} \operatorname{sgn}^{t}, p+t\right) \rtimes \mathbf{1}, \\
& \operatorname{Im} B_{3}(p) \simeq \zeta\left(||^{(t-p) / 2} \operatorname{sgn}^{p}, p+t\right) \rtimes \mathbf{1} .
\end{aligned}
$$
ding:

Next, applying Theorem 2.5 and $B_{2}(t)^{-1}$, we obtain the following embed-

$$
\begin{aligned}
& a: \zeta\left(||^{(p+t) / 2} \operatorname{sgn}^{t+1}, p-t\right) \rtimes \mathbf{1} \hookrightarrow||^{t} \operatorname{sgn}^{t+1} \times \mid{ }^{p} \operatorname{sgn}^{p} \rtimes \mathbf{1}, \\
& b: \delta\left(||^{(t-p) / 2} \operatorname{sgn}^{t}, p+t\right) \rtimes \mathbf{1} \hookrightarrow||^{t} \operatorname{sgn}^{t+1} \times||^{p} \operatorname{sgn}^{p} \rtimes \mathbf{1} .
\end{aligned}
$$

We claim

$$
\operatorname{Im} a \cap \operatorname{Im} b \simeq \operatorname{Lang}\left(\delta\left(||^{(t-p) / 2} \operatorname{sgn}^{t}, p+t\right) \rtimes \mathbf{1}\right) .
$$

First, we show that $\operatorname{Im} a \cap \operatorname{Im} b \neq 0$. If not, then, since by Theorem 2.5 we have an epimorphism

$$
||^{t} \operatorname{sgn}^{t+1} \times||^{p} \operatorname{sgn}^{p} \rtimes \mathbf{1} \rightarrow \delta\left(||^{(p+t) / 2} \operatorname{sgn}^{t+1}, p-t\right) \rtimes \mathbf{1},
$$

having kernel equal to $\operatorname{Im} a$, we obtain

$$
\begin{aligned}
\delta\left(||^{(t-p) / 2} \operatorname{sgn}^{t}, p+t\right) \rtimes \mathbf{1} & \hookrightarrow \delta\left(||^{(p+t) / 2} \operatorname{sgn}^{t+1}, p-t\right) \rtimes \mathbf{1} \\
& =\delta\left(||^{(p+t) / 2} \operatorname{sgn}^{t}, p-t\right) \rtimes \mathbf{1} .
\end{aligned}
$$

Now, the composition series of $\delta\left(||^{(p+t) / 2} \operatorname{sgn}^{t}, p-t\right) \rtimes \mathbf{1}$ described by Theorem 10.3 shows

$$
\operatorname{Lang}\left(\delta\left(||^{(p-t) / 2} \operatorname{sgn}^{t}, p+t\right) \rtimes \mathbf{1}\right) \hookrightarrow \delta\left(||^{(p-t) / 2} \operatorname{sgn}^{t}, p+t\right) \rtimes \mathbf{1} .
$$

This is a contradiction since $\delta\left(||^{(p-t) / 2} \operatorname{sgn}^{t}, p+t\right) \rtimes \mathbf{1}$ is reducible (see Theorem 10.1). This shows that the right-hand side of (11.2) is a subrepresentation of the left-hand side. To see equality, we apply Theorem 10.1 that implies that other two irreducible subquotients $X(p,-t)$ and $X(t,-p)$, being large are not subquotients of $\zeta\left(||^{(p+t) / 2} \operatorname{sgn}^{t+1}, p-t\right) \rtimes \mathbf{1}$. This proves (11.2), but it also proves

$$
\begin{aligned}
& \zeta\left(||^{(p+t) / 2} \operatorname{sgn}^{t+1}, p-t\right) \rtimes \mathbf{1} \geq X(p,-t), X(t,-p), \\
& \operatorname{Lang}\left(\delta\left(||^{(p-t) / 2} \operatorname{sgn}^{t}, p+t\right) \rtimes \mathbf{1}\right) \hookrightarrow \zeta\left(||^{(p+t) / 2} \operatorname{sgn}^{t+1}, p-t\right) \rtimes \mathbf{1} .
\end{aligned}
$$


Next, clearly (11.1) implies $\operatorname{Im} B_{1}(p) \circ i \subset \operatorname{Im} a$. Also, (11.1) implies $\operatorname{Im} i \not \subset$ $\operatorname{ker} B_{1}(p)$. Hence $\operatorname{Im} B_{1}(p) \circ i \neq 0$. Now, if $\operatorname{Im} B_{1}(p) \circ i \cap \operatorname{Im} b \neq 0$, then we prove

$$
\operatorname{Im} B_{1}(p) \circ i \cap \operatorname{Im} b \simeq \operatorname{Lang}\left(\delta\left(||^{(t-p) / 2} \operatorname{sgn}^{t}, p+t\right) \rtimes \mathbf{1}\right)
$$

in the same way we have proved (11.2). Written differently, we obtain

$$
\operatorname{Im} B_{2}(p) \circ B_{1}(p) \circ i \cap \operatorname{ker} B_{3}(p) \simeq \operatorname{Lang}\left(\delta\left(||^{(t-p) / 2} \operatorname{sgn}^{t}, p+t\right) \rtimes \mathbf{1}\right) .
$$

Hence, the commutative diagram (11.1) implies following exact sequence:

$$
\begin{aligned}
\operatorname{Lang}\left(\delta\left(||^{(p-t) / 2} \operatorname{sgn}^{t}, p+t\right) \rtimes \mathbf{1}\right) \hookrightarrow \operatorname{Im} B_{1}(p) \circ i \\
\quad \rightarrow \operatorname{Lang}\left(||^{p} \operatorname{sgn}^{p} \rtimes X(t,+)\right) \oplus \operatorname{Lang}\left(||^{p} \operatorname{sgn}^{p} \rtimes X(p,-)\right),
\end{aligned}
$$

if $\operatorname{Im} B_{1}(p) \circ i \cap \operatorname{Im} b \neq 0$. Next, we note that

$$
||^{p} \operatorname{sgn}^{p} \rtimes V_{t}\left\{\begin{array}{l}
\geq \operatorname{Lang}\left(\delta\left(||^{(p+t) / 2} \operatorname{sgn}^{t}, p-t\right) \rtimes \mathbf{1},(\text { multiplicity one }),\right. \\
⿱ \operatorname{Lang}\left(||^{t} \operatorname{sgn}^{t} \rtimes X(p, \pm)\right), \operatorname{Lang}\left(\delta\left(||^{(p-t) / 2} \operatorname{sgn}^{t}, p+t\right) \rtimes \mathbf{1}\right), \\
\geq X(p, t), X(p,-t), X(t,-p), X(-t,-p) .
\end{array}\right.
$$

Hence, Theorem 10.3, (11.3) and (9.5) imply that $\operatorname{Lang}\left(\delta\left(||^{(t-p) / 2} \operatorname{sgn}^{t}, p+\right.\right.$ $t) \rtimes \mathbf{1})$ must appear in $\operatorname{Im} i$ at least twice. Since it appears exactly once in ker $B_{1}(p)$ (see Theorem 10.3), it must appear in $B_{1}(p) \circ i$. Hence (11.4) holds without restrictions.

To prove the first inequality in (11.5), we consider another commutative diagram (see (7.1)):

$$
\begin{aligned}
& \delta\left(||^{(p+t) / 2} \operatorname{sgn}^{t+1}, p-t\right) \rtimes \mathbf{1} \stackrel{i_{1}}{\longrightarrow}||^{p} \operatorname{sgn}^{p} \times||^{t} \operatorname{sgn}^{t+1} \rtimes \mathbf{1} \\
&||^{p} \operatorname{sgn}^{p} \times||^{-t} \operatorname{sgn}^{t+1} \rtimes \mathbf{1} \\
& A_{2} \downarrow \\
&||^{-t} \operatorname{sgn}^{t+1} \times||^{p} \operatorname{sgn}^{p} \rtimes \mathbf{1} \\
& A_{3} \simeq \downarrow \\
& \delta\left(||^{-(p+t) / 2} \operatorname{sgn}^{t+1}, p-t\right) \rtimes \mathbf{1} \stackrel{j_{1}}{\longrightarrow}||^{-t} \operatorname{sgn}^{t+1} \times||^{-p} \operatorname{sgn}^{p} \rtimes \mathbf{1} \\
& A_{4} \neq 0 \text { (induced) } \mid \\
&||^{-p} \operatorname{sgn}^{p} \times||^{-t} \operatorname{sgn}^{t+1} \rtimes \mathbf{1}
\end{aligned}
$$

This diagram consists of holomorphic and non-zero operators (see Lemma 7.2). This implies

$$
||^{p} \operatorname{sgn}^{p} \rtimes V_{t} \geq \operatorname{Lang}\left(\delta\left(||^{(p+t) / 2} \operatorname{sgn}^{t+1}, p-t\right) \rtimes \mathbf{1}\right) .
$$


Now, we complete the proof of (11.5). First, we combine Lemma 6.1 and Corollary 10.2(v) for $\operatorname{Lang}\left(||^{t} \operatorname{sgn}^{t} \rtimes X(p, \pm)\right)$, and Lemma 6.1 and description of the types of discrete series for $X(p, t)$ and $X(-t,-p)$. Next, Theorem 10.1 implies that $X(p,-t)$ and $X(t,-p)$ are large and this proves (11.5) for them. Finally, we note that the type $(p,-t-1)$ appears with multiplicity one in $\operatorname{Lang}\left(\delta\left(||^{(p-t) / 2} \operatorname{sgn}^{t}, p+t\right) \rtimes \mathbf{1}\right)$ (by Corollary $10.2(\mathrm{v})$ ), with multiplicity $t+1$ in $\delta\left(||^{(p+t) / 2} \operatorname{sgn}^{t+1}, p-t\right) \rtimes \mathbf{1}$ (by Lemma 6.1), with multiplicity zero in $\mid{ }^{t} \operatorname{sgn}^{t} \rtimes X(p, \pm)$, and with multiplicity $t$ in ||$^{p} \operatorname{sgn}^{p} \rtimes V_{t}$. Now, Theorem 10.3 implies that this type appears in $\operatorname{Lang}\left(\delta\left(||^{(p+t) / 2} \operatorname{sgn}^{t+1}, p-t\right) \rtimes \mathbf{1}\right)$ with multiplicity $t$. This and (11.7) show that $\operatorname{Lang}\left(\delta\left(||^{(p-t) / 2} \operatorname{sgn}^{t}, p+t\right) \rtimes \mathbf{1}\right)$ do not appear in ||$^{p} \operatorname{sgn}^{p} \rtimes V_{t}$, while $\operatorname{Lang}\left(\delta\left(||^{(p+t) / 2} \operatorname{sgn}^{t+1}, p-t\right) \rtimes \mathbf{1}\right)$ appears with multiplicity at most one as claimed. This proves (11.5).

Next, we note that $\operatorname{Im} i_{1}=\operatorname{ker} B_{1}(p)$ and $\operatorname{Im} i=\operatorname{ker} A_{1}$, and we claim the following:

$$
\begin{aligned}
& \operatorname{Im} i \cap \operatorname{ker} B_{1}(p) \simeq \\
& \text { maximal proper subrepresentation of } \delta\left(||^{(p+t) / 2} \operatorname{sgn}^{t+1}, p-t\right) \rtimes \mathbf{1} .
\end{aligned}
$$

This follows directly from (11.5) and Theorem 10.3 since $\operatorname{Im} A_{1} \simeq \mid{ }^{p} \operatorname{sgn}^{p} \rtimes V_{t}$. Now, since

$$
\begin{aligned}
& \operatorname{ker} A_{2} \simeq \delta\left(||^{(p-t) / 2} \operatorname{sgn}^{t+1}, p+t\right) \rtimes \mathbf{1}, \\
& \operatorname{ker} A_{4} \simeq \delta\left(||^{-(p+t) / 2} \operatorname{sgn}^{t+1}, p-t\right) \rtimes \mathbf{1},
\end{aligned}
$$

and the composition $A_{4} \circ A_{3} \circ A_{2} \circ A_{1}$ has image $\operatorname{Lang}\left(||^{p} \operatorname{sgn}^{p} \times||^{t} \operatorname{sgn}^{t+1} \rtimes \mathbf{1}\right)$, (11.5) implies (i).

Next, Theorem 10.3, (9.4) and (9.8) imply that in $\mathcal{R}(S p(4, \mathbb{R}))$ we have the following:

$$
\begin{aligned}
&||^{p} \operatorname{sgn}^{p} \rtimes X(t,+)+||^{p} \operatorname{sgn}^{p} \rtimes X(t,-) \\
&= X(p,-t)+X(t,-p)+2 \cdot \operatorname{Lang}\left(\delta\left(||^{(p-t) / 2} \operatorname{sgn}^{t}, p+t\right) \rtimes \mathbf{1}\right) \\
&+\operatorname{Lang}\left(||^{t} \operatorname{sgn}^{t} \rtimes X(p,+)\right)+\operatorname{Lang}\left(||^{t} \operatorname{sgn}^{t} \rtimes X(p,-)\right) \\
&+\operatorname{Lang}\left(||^{p} \operatorname{sgn}^{p} \rtimes X(t,+)\right)+\operatorname{Lang}\left(||^{p} \operatorname{sgn}^{p} \rtimes X(t,-)\right) .
\end{aligned}
$$

Now, we use the types to write this more precisely. We have that $(p+$ $1,-t),(t,-p-1),(p+1, t),(-t,-p-1)$, and $(p,-t-1)$ appear in $X(p,-t), X(t,-p), \operatorname{Lang}\left(||^{t} \operatorname{sgn}^{t} \rtimes X(p,+)\right), \operatorname{Lang}\left(||^{t} \operatorname{sgn}^{t} \rtimes X(p,-)\right)$, and $\operatorname{Lang}\left(\delta\left(||^{(p-t) / 2} \operatorname{sgn}^{t}, p+t\right) \rtimes \mathbf{1}\right)$ with multiplicity one, respectively (by (dis) in Section 8 and Corollary $10.2(\mathrm{v}))$. Lemma 6.1 shows that $(p+1,-t)$ and $(p+1, t)$ belong to $\mid{ }^{p} \operatorname{sgn}^{p} \rtimes X(t,+)$ but not to ||$^{p} \operatorname{sgn}^{p} \rtimes X(t,-)$. Also, $(t,-p-1)$ and $(-t,-p-1)$ belong to ||$^{p} \operatorname{sgn}^{p} \rtimes X(t,-)$ but not to ||$^{p} \operatorname{sgn}^{p} \rtimes X(t,+)$. Finally, $(p,-t-1)$ appears in ||$^{p} \operatorname{sgn}^{p} \rtimes X(t,-)$ and in ||$^{p} \operatorname{sgn}^{p} \rtimes X(t,-)$ with multiplicity one and with multiplicity at most one, 
respectively. Now, the composition series of $\delta\left(||^{(p+t) / 2} \operatorname{sgn}^{t}, p-t\right) \rtimes \mathbf{1}$ given by Theorem 10.3, (11.4) and (11.8) imply (ii) and (iii).

Now, (i), (ii) and (iii), together with (9.5) and Theorems 10.1 and 10.3, show that in semisimplification (iv) and (v) hold. Using (11.1) we see that $\zeta\left(||^{(p+t) / 2} \operatorname{sgn}^{t+1}, p-t\right) \rtimes \mathbf{1}$ and $\operatorname{Lang}\left(||^{p} \operatorname{sgn}^{p} \times \mid{ }^{t} \operatorname{sgn}^{t+1} \rtimes \mathbf{1}\right)$ are both quotients of ||$^{p} \operatorname{sgn}^{p} \times||^{t} \operatorname{sgn}^{t+1} \rtimes \mathbf{1}$. Now, (11.4) completes the proof of (iv). In order to prove (v), we note the following exact sequence (which follows from Theorem 2.5 taking contragredients):

$$
\begin{aligned}
\widetilde{\delta}\left(||^{(p+t) / 2} \operatorname{sgn}^{t+1}, p-t\right) \rtimes \mathbf{1} & \hookrightarrow||^{-t} \operatorname{sgn}^{t+1} \times||^{-p} \operatorname{sgn}^{p} \rtimes \mathbf{1} \\
& \rightarrow \widetilde{\zeta}\left(||^{(p+t) / 2} \operatorname{sgn}^{t+1}, p-t\right) \rtimes \mathbf{1} .
\end{aligned}
$$

Using the Langlands classification and the description of contragredient representations (see the end of the proof of Theorem 10.1): $\widetilde{X(p,-)} \simeq X(p,+)$, $\widetilde{X(p, t)} \simeq X(-t,-p)$, and $\widetilde{X(p,-t)} \simeq X(t,-p)$, we obtain the following description of contragredient representations:

$$
\begin{aligned}
& \widetilde{\operatorname{Lang}}\left(||^{p} \operatorname{sgn}^{p} \rtimes X(t,+)\right) \simeq \operatorname{Lang}\left(||^{p} \operatorname{sgn}^{p} \rtimes X(t,-)\right), \\
& \widetilde{\operatorname{Lang}}\left(||^{p} \operatorname{sgn}^{p} \rtimes X(t,-)\right) \simeq \operatorname{Lang}\left(||^{p} \operatorname{sgn}^{p} \rtimes X(t,+)\right), \\
& \widetilde{\operatorname{Lang}}\left(||^{t} \operatorname{sgn}^{t} \rtimes X(p,+)\right) \simeq \operatorname{Lang}\left(||^{t} \operatorname{sgn}^{t} \rtimes X(p,-)\right), \\
& \widetilde{\operatorname{Lang}}\left(||^{t} \operatorname{sgn}^{t} \rtimes X(p,-)\right) \simeq \operatorname{Lang}\left(||^{t} \operatorname{sgn}^{t} \rtimes X(p,+)\right), \\
& \widetilde{\operatorname{Lang}}\left(\delta\left(||^{(p-t) / 2} \operatorname{sgn}^{t}, p+t\right) \rtimes \mathbf{1}\right) \simeq \operatorname{Lang}\left(\delta\left(||^{(p-t) / 2} \operatorname{sgn}^{t}, p+t\right) \rtimes \mathbf{1}\right), \\
& \widetilde{\operatorname{Lang}}\left(\delta\left(||^{(p+t) / 2} \operatorname{sgn}^{t}, p-t\right) \rtimes \mathbf{1}\right) \simeq \operatorname{Lang}\left(\delta\left(||^{(p+t) / 2} \operatorname{sgn}^{t}, p-t\right) \rtimes \mathbf{1}\right), \\
& \widetilde{\operatorname{Lang}}\left(||^{p} \operatorname{sgn}^{p} \times||^{t} \operatorname{sgn}^{t+1} \rtimes \mathbf{1}\right) \simeq \operatorname{Lang}\left(||^{p} \operatorname{sgn}^{p} \times||^{t} \operatorname{sgn}^{t+1} \rtimes \mathbf{1}\right) .
\end{aligned}
$$

Now, applying Theorem 10.3 we obtain the filtration of $\widetilde{\delta}\left(||^{(p+t) / 2} \operatorname{sgn}^{t+1}, p-\right.$ $t) \rtimes \mathbf{1}$ and applying (iv) we obtain the filtration of $\widetilde{\zeta}\left(||^{(p+t) / 2} \operatorname{sgn}^{t+1}, p-\right.$ $t) \rtimes \mathbf{1}$. Combining them with (11.9), we obtain the filtration of the induced representation:

$$
||^{-t} \operatorname{sgn}^{t+1} \times||^{-p} \operatorname{sgn}^{p} \rtimes \mathbf{1} .
$$

But, we note that $\zeta\left(||^{(p-t) / 2} \operatorname{sgn}^{t+1}, p+t\right) \rtimes \mathbf{1}$ is a subrepresentation of ||$^{-t} \operatorname{sgn}^{t+1} \times||^{p} \operatorname{sgn}^{p} \rtimes \mathbf{1}$ (see Theorem 2.5). Next, Theorem 2.4 implies

$$
||^{-t} \operatorname{sgn}^{t+1} \times||^{p} \operatorname{sgn}^{p} \rtimes \mathbf{1} \simeq||^{-t} \operatorname{sgn}^{t+1} \times||^{-p} \operatorname{sgn}^{p} \rtimes \mathbf{1} .
$$

Hence $\widetilde{\zeta}\left(||^{(p-t) / 2} \operatorname{sgn}^{t+1}, p+t\right) \rtimes \mathbf{1}$ appears as a quotient of (11.10). Projecting to the filtration of (11.10) to $\widetilde{\zeta}\left(||^{(p-t) / 2} \operatorname{sgn}^{t+1}, p+t\right) \rtimes \mathbf{1}$, we easily obtain (v) since we already know the semisimplification of $\zeta\left(||^{(p-t) / 2} \operatorname{sgn}^{t+1}, p+t\right) \rtimes \mathbf{1}$.

Finally, we need to consider the degenerate case. 
THEOREM 11.2. Assume $p>t=0$. Then we have the following:

(i) ||$^{p} \operatorname{sgn}^{p} \rtimes X(0,+)$ has the following filtration $V_{0} \subset V_{1} \subset V_{2} \simeq||^{p} \operatorname{sgn}^{p} \rtimes$ $X(0,+)$, where

$$
\begin{aligned}
& V_{0} \simeq X^{2}(p, 0), \\
& V_{1} / V_{0} \simeq \operatorname{Lang}\left(\delta\left(||^{p / 2}, p\right) \rtimes \mathbf{1}\right), \\
& V_{2} / V_{1} \simeq \operatorname{Lang}\left(||^{p} \operatorname{sgn}^{p} \rtimes X(0,+)\right) .
\end{aligned}
$$

(ii) ||$^{p} \operatorname{sgn}^{p} \rtimes X(0,-)$ has the following filtration $V_{0} \subset V_{1} \simeq||^{p} \operatorname{sgn}^{p} \rtimes$ $X(0,-)$, where

$$
\begin{aligned}
& V_{0} \simeq X^{1}(0,-p), \\
& V_{1} / V_{0} \simeq \operatorname{Lang}\left(\delta\left(||^{p / 2}, p\right) \rtimes \mathbf{1}\right), \\
& V_{2} / V_{1} \simeq \operatorname{Lang}\left(||^{p} \operatorname{sgn}^{p} \rtimes X(0,-)\right) .
\end{aligned}
$$

(iii) $\zeta\left(||^{p / 2} \operatorname{sgn}, p\right) \rtimes \mathbf{1}$ has the following filtration $V_{0} \subset V_{1} \subset V_{2} \simeq$ $\zeta\left(||^{p / 2} s g n, p\right) \rtimes \mathbf{1}$, where

$$
\begin{aligned}
& V_{0} \simeq \operatorname{Lang}\left(\delta\left(||^{p / 2}, p\right) \rtimes \mathbf{1}\right), \\
& V_{1} / V_{0} \simeq \operatorname{Lang}\left(||^{p} \operatorname{sgn}^{p} \rtimes X(0,+)\right) \oplus \operatorname{Lang}\left(||^{p} \operatorname{sgn}^{p} \rtimes X(0,-)\right) .
\end{aligned}
$$

Proof. This has the proof that is similar to the proof of Theorem 11.1. We leave to the reader to make necessary adjustments (one may also proceed applying translations functors as in the proof of Theorem 10.7).

\section{COMPOSITION SERIES IN THE CASE OF NON-INTEGRAL INFINITESIMAL CHARACTER}

In this section we complete our analysis of the composition series of generalized and degenerate principal series for $S p(4, \mathbb{R})$.

TheOrEm 12.1. Assume that $\eta=||^{s} \operatorname{sgn}^{\epsilon}(s \in \mathbb{C}, \epsilon \in\{0,1\})$ and $p \in \mathbb{Z}_{\geq 0}$. Then we have the following:

(i) Assume $s-k / 2 \notin \mathbb{Z}$ (hence, the infinitesimal character $(s+k / 2, s-k / 2)$ of $\delta(\eta, k) \rtimes \mathbf{1}$ and $\zeta(\eta, k) \rtimes \mathbf{1}$ is not integral). Then $\delta(\eta, k) \rtimes \mathbf{1}$ and $\zeta(\eta, k) \rtimes \mathbf{1}$ are irreducible.

(ii) Assume $s \notin \mathbb{Z}$ (hence, the infinitesimal character $(s, p)$ of $\eta \rtimes X(p, \pm$ ) and $\eta \rtimes V_{p}$ (exists only for $p>0$ ) is not integral). Then $\eta \rtimes X(p, \pm)$ and $\eta \rtimes V_{p}$ (exists only for $p>0$ ) are irreducible.

Proof. We prove (i). The proof of (ii) is analogous and it is left to the reader. To prove (i), we note that the contragredients of $\delta(\eta, k) \rtimes \mathbf{1}$ and $\zeta(\eta, k) \rtimes \mathbf{1}$ are given by Lemma 7.1. Hence we may assume $\operatorname{Re}(s) \geq 0$. To prove irreducibility of $\delta(\eta, k) \rtimes \mathbf{1}$, we first consider the case $\operatorname{Re}(s)>0$. Now, we use the notation introduced in Lemma 7.2. Applying Theorems 2.4 and 2.5 we see that all intertwining operators $A_{i}(t) i=1,2,3$, are holomorphic and 
isomorphisms. Hence (7.1) shows that $A(t)$ is an isomorphism. This implies that $\delta(\eta, k) \rtimes \mathbf{1}$ is irreducible. If $\operatorname{Re}(s)=0$, then $\delta(\eta, k) \rtimes \mathbf{1}$ is irreducible by Lemma 8.1.

To prove reducibility of $\zeta(\eta, k) \rtimes \mathbf{1}$, for $t=R e(s) \geq 0$, we use some nonzero (induced) intertwining operators $A_{1}, A_{2}, A_{3}$ instead of $A_{1}(t), A_{2}(t), A_{3}(t)$, respectively, in (7.1). Now, since $\delta(\eta, k) \rtimes \mathbf{1}$ is irreducible it is large. In fact, it is a unique large subquotient of

$$
\eta_{u}||^{t+k / 2} \operatorname{sgn}^{k+1} \times \eta_{u}||^{t-k / 2} \rtimes \mathbf{1}=\delta(\eta, k) \rtimes \mathbf{1}+\zeta(\eta, k) \rtimes \mathbf{1} .
$$

This implies that there exists a non-zero intertwining operator $A$ such that the analogue of (7.1) holds. Now, we consider the two cases:

First, assume that $t=R e(s) \geq k / 2$. Then we add an (induced) non-zero intertwining operator:

$$
\begin{aligned}
\eta_{u}^{-1}||^{-t+k / 2} \times \eta_{u}^{-1}||^{-t-k / 2} \operatorname{sgn}^{k+1} \rtimes \mathbf{1} \\
\stackrel{A_{4}}{\longrightarrow} \eta_{u}^{-1}||^{-t-k / 2} \operatorname{sgn}^{k+1} \times \eta_{u}^{-1}||^{-t+k / 2} \rtimes \mathbf{1}
\end{aligned}
$$

to the diagram (7.1). Now, the composition $A_{4} \circ A_{3} \circ A_{2} \circ A_{1}$ is isomorphic to

$$
\operatorname{Lang}\left(\eta_{u}||^{t+k / 2} \operatorname{sgn}^{k+1} \times \eta_{u}||^{t-k / 2} \rtimes \mathbf{1}\right) .
$$

It is clear that we have the following exact sequence:

$$
\delta(\eta, k) \rtimes \mathbf{1} \simeq \operatorname{ker} A_{4}=\operatorname{Im} j \hookrightarrow \operatorname{Im} A_{3} \circ A_{2} \circ A_{1} \rightarrow \zeta(\eta, k) \rtimes \mathbf{1} .
$$

Hence

$$
\zeta(\eta, k) \rtimes \mathbf{1} \simeq \operatorname{Lang}\left(\eta_{u}||^{t+k / 2} \operatorname{sgn}^{k+1} \times \eta_{u}||^{t-k / 2} \rtimes \mathbf{1}\right) .
$$

Next, we assume that $0 \leq t=\operatorname{Re}(s)<k / 2$. Then, except the operator $A_{4}$, we need to add one more isomorphism:

$$
\begin{aligned}
\eta_{u}^{-1}||^{-t-k / 2} \operatorname{sgn}^{k+1} \times \eta_{u}^{-1}||^{-t+k / 2} \rtimes \mathbf{1} \\
\stackrel{A_{5} \simeq}{\longrightarrow} \eta_{u}^{-1}||^{-t-k / 2} \operatorname{sgn}^{k+1} \times \eta_{u}||^{t-k / 2} \rtimes \mathbf{1} .
\end{aligned}
$$

Now, $A_{5} \circ A_{4} \circ A_{3} \circ A_{2} \circ A_{1}$ is isomorphic to $\operatorname{Lang}\left(\eta_{u}||^{t+k / 2} \operatorname{sgn}^{k+1} \times\right.$ $\left.\eta_{u}||^{-t+k / 2} \rtimes \mathbf{1}\right)$. Also, (12.1) holds and we obtain

$$
\zeta(\eta, k) \rtimes \mathbf{1} \simeq \operatorname{Lang}\left(\eta_{u}||^{t+k / 2} \operatorname{sgn}^{k+1} \times \eta_{u}^{-1}||^{-t+k / 2} \rtimes \mathbf{1}\right) .
$$

This completes the proof of (i).

\section{ACKNOWLEDGEMENTS.}

I would like to thank D. Miličić, G. Savin, M. Tadić, D. Vogan for useful discussions related to the paper. The work on this project started during our stay at Paris VII in May of 2004. I would like to thank Corinne Blondel for inviting me there and for her warm hospitality. Final version of the paper was written while I was a member of the Erwin Scrödinger Institute in Vienna in fall of 2008. I would like to thank Schwermer for his hospitality. 


\section{REFERENCES}

[1] A. M. Aubert, Dualité dans le groupe de Grothendieck de la catégorie des représentations lisses de longueur finie d'un groupe réductif p-adique, Trans. Amer. Math. Soc. 347 (1995), 2179-2189 (and Erratum, Trans. Amer. Math. Soc 348 (1996), 4687-4690.)

[2] J. Arthur, Intertwining operators and residues. I. Weighted characters, J. Funct. Anal. 84 (1989), 19-84.

[3] N. Bourbaki, Groupe et Algébres de Lie, Chap. 6, Masson, Paris, 1981.

[4] D. Goldberg, Reducibility of induced representations for $S p(2 n)$ and $S O(n)$, Amer. J. Math. 116 (1994), 1101-1151.

[5] R. Herb, Elliptic representations for $S p(2 n)$ and $S O(n)$, Pacific J. Math. 161 (1993), 347-358.

[6] C. Jantzen, H. Kim, Parametrization of the image of normalized intertwining operators, Pacific J. Math. 199 (2001), 367-415.

[7] H. Kim, The residual spectrum of $\mathrm{Sp}_{4}$, Compositio Math. 99 (1995), 129-151.

[8] H. Kim, The residual spectrum of $G_{2}$, Canad. J. Math. 48 (1996), 1245-1272.

[9] H. Kim, Residual spectrum of odd orthogonal groups, Internat. Math. Res. Notices 17 (2001), 873-906

[10] A. W. Knapp, Representation theory of semisimple groups. An overview based on examples, Princeton Mathematical Series 36, Princeton University Press, Princeton, NJ, 1986.

[11] A. W. Knapp, D. Vogan, Cohomological induction and unitary representations, Princeton Mathematical Series 45, Princeton University Press, Princeton, NJ, 1995.

[12] D. Miličić, Algebraic D-modules and representation theory of semisimple Lie groups, Contemp. Math. 154, Amer. Math. Soc., Providence, RI, 1993, 133-168.

[13] C. Møglin, Orbites unipotentes et spectre discret non ramifie: le cas des groupes classiques dèployès, Compositio Math. 77 (1991), 1-54.

[14] C. Møglin, Reprèsentations unipotentes et formes automorphes de carrè intègrable, Forum Math. 6 (1994), 651-744

[15] C. Møglin, J.L. Waldspurger, Le spectre résiduel de $G L(n)$, Ann. Sci. École Norm. Sup. (4) 22 (1989), 605-674.

[16] C. Møglin, J.L. Waldspurger, Spectral decomposition and Eisenstein series, Cambridge Tracts in Mathematics 113, Cambridge University Press, Cambridge, 1995.

[17] G. Muić, The unitary dual of $p$-adic $G_{2}$, Duke Math. J. 90 (1997), 465-493.

[18] G. Muić, Reducibility of generalized principal series, Canad. J. Math. 57 (2005), 616-647.

[19] G. Muić, Composition series of generalized principal series; the case of strongly positive discrete series, Israel J. Math 140 (2004), 157-202.

[20] G. Muić, Reducibility of standard representations, Pacific J. Math. 222 (2005), 133168.

[21] G. Muić, On certain classes of unitary representations for split classical groups, Canad. J. Math. 59 (2007), 148-185.

[22] G. Muić, Some applications of degenerate Eisenstein series on $\mathrm{Sp}_{2 n}$, J. Ramanujan Math. Soc. 23, No.3 (2008) 222-257.

[23] G. Muić, M. Tadić, Unramified Unitary Duals for Split Classical p-adic Groups; The topology and Isolated Representations, Shahidi's volume (to appear).

[24] P. J. Sally, M. Tadić, Induced representations and classifications for $G S p(2, F)$ and $\operatorname{Sp}(2, F)$, Mém. Soc. Math. France (N.S.) 52 (1993), 75-133.

[25] F. Shahidi, Local coefficients as Artin factors for real groups, Duke Math. J. 52 (1985), 973-1007. 
[26] B. Speh, Unitary representations of $G L(n, \mathbb{R})$ with nontrivial $(\mathfrak{g}, K)$-cohomology, Invent. Math. 71 (1983), 443-465.

[27] B. Speh, D. A. Vogan, Reducibility of generalized principal series representations, Acta. Math. 145 (1980), 227-299.

[28] M. Tadić, On reducibility of parabolic induction, Israel J. Math. 107 (1998), 29-91.

[29] D. A. Vogan, Gelfand-Kirillov dimension for Harish-Chandra modules, Invent. Math. 48 (1978), 75-98.

[30] D. A. Vogan, Representations of real reductive Lie groups, Progress in Mathematics 15, Birkhäuser, Boston, 1981.

[31] D. A. Vogan, The unitary dual of $G_{2}$, Invent. Math. 116 (1994), 677-791.

[32] T. Watanabe, Residual spectrum representations of $S p_{4}$, Nagoya Math J. 127 (1992), $15-47$.

[33] G. Zuckerman, Tensor products of finite and infinite dimensional representations of semisimple Lie groups, Ann. Math. (2) 106 (1977), 295-308.

[34] A. V. Zelevinsky, Induced representations of reductive p-adic groups. On irreducible representations of $G L(n)$, Ann. Sci. École Norm. Sup. (4) 13 (1980), 165-210.

\section{G. Muić}

Department of Mathematics

University of Zagreb

10000 Zagreb

Croatia

E-mail: gmuic@math.hr

Received: 23.6.2008.

Revised: 12.11.2008. 